\title{
Weyl covariance, and proposals for superconformal prepotentials in 10D superspaces
}

\section{S. James Gates Jr., Yangrui Hu and S.-N. Hazel Mak}

Brown Theoretical Physics Center, Box S, 340 Brook Street, Barus Hall, Providence, RI 02912, U.S.A.

Department of Physics, Brown University,

Box 1843, 182 Hope Street, Barus 8 Holley, Providence, RI 02912, U.S.A.

E-mail: sylvester_gates@brown.edu, yangrui_hu@brown.edu, sze_ning_mak@brown.edu

AbStRact: Proposals are made to describe the Weyl scaling transformation laws of supercovariant derivatives $\nabla_{\underline{A}}$, the torsion supertensors $T_{\underline{A}} \underline{B} \underline{C}$, and curvature supertensors $R_{\underline{A}} \underline{B} \underline{\underline{c}} \underline{\underline{d}}$ in $10 \mathrm{D}$ superspaces. Starting from the proposal that an unconstrained supergravity prepotential for the $11 \mathrm{D}, \mathcal{N}=1$ theory is described by a scalar superfield, considerations for supergravity prepotentials in the 10D theories are enumerated. We derive infinitesimal $10 \mathrm{D}$ superspace Weyl transformation laws and discover ten possible $10 \mathrm{D}, \mathcal{N}=1$ superfield supergravity prepotentials. The first identification of all off-shell ten dimensional supergeometrical Weyl field strength tensors, constructed from respective torsions, is presented.

KEYWORDS: Supergravity Models, Superspaces

ArXiv ePrint: 2007.05097 


\section{Contents}

1 Introduction 1

2 Review of $11 \mathrm{D}, \mathcal{N}=1$ supergravity derivatives \& scale transformations 4

3 Supergravity derivatives \& scale transformations in 10D 10

$3.110 \mathrm{D}, \mathcal{N}=$ IIA supergravity derivatives \& scale transformations $\quad 10$

$3.210 \mathrm{D}, \mathcal{N}=$ IIB supergravity derivatives \& scale transformations $\quad 13$

3.3 10D, $\mathcal{N}=1$ supergravity derivatives \& scale transformations 18

4 A new methodology: Adynkras \& ADA scans $\quad 19$

4.1 Component Dynkin Label examples \& ADA scans 22

5 Toward the rest of the story $\quad 23$

6 Conclusion 27

A 10D Weyl scaling properties of weight $\geq \frac{3}{2}$ super tensors $\quad 28$

$\begin{array}{lll}\text { A. } 10 \mathrm{D}, \mathcal{N}=2 \mathrm{~A} & 28\end{array}$

$\begin{array}{lll}\text { A. } 210 \mathrm{D}, \mathcal{N}=2 \mathrm{~B} & 29\end{array}$

A.3 $10 \mathrm{D}, \mathcal{N}=1 \quad 30$

$\begin{array}{lr}\text { B Adynkra libraries } & \mathbf{3 0}\end{array}$

B.1 Dynkin Label library of $\mathcal{V}_{[0,0,1,0,0]} 30$

B.2 Dynkin Label library of $\mathcal{V}_{[1,0,1,0,1]} \quad 32$

B.3 Dynkin Label library of $\mathcal{V}_{[3,0,0,0,1]} \quad 35$

B.4 Dynkin Label library of $\mathcal{V}_{[4,0,0,0,0]} 38$

\section{Introduction}

The first explicit discussions in the literature on the topic of Weyl symmetry in superspace were initiated among the works in $[1,2]$ by Howe et al. . The subject of the interplay between conformal symmetry and the constraints of superspace descriptions of Poincaré supergravity, has long been of fascination to one of the authors [3-7]. Of course, numbers of other authors have also pursued this subject. A special area of these considerations involves the context of $11 \mathrm{D}, \mathcal{N}=1$ superspace [8, 9] related to M-Theory [10]. After its beginning, a literature (e.g. [11-19]) has been built up including discussions in superspace and also at the level of component fields. These are but a small selection and the interested readers should look at the references in these works for a more complete listing of such works.

Near the end of a 1996 investigation [20], the following paragraph can be found:

For although we believe our observation is important, we know of at least two arguments that suggest that there must exist at least one other tensor superfield that will be required to have a completely off-shell formalism. This is to be 
expected even from the structure of the non-minimal $4 D, \mathcal{N}=1$ supergravity. There it is known that there are three algebraically independent tensors $W_{\alpha \beta \gamma}$, $G_{\underline{a}}$ and $T_{\alpha}$, so apparently it remains to find the eleven dimensional analog of the $G_{\underline{a}}$. In future works, these aspects of the eleven dimensional theory will require further study.

From our present perspective, the "strong form" of the constraints given in a 2000 work by Cederwall, Gran, Nielsen, and Nilsson [12], appears to provide the solution of our 1996 dilemma. These authors introduced a dimension zero tensor $X_{[5]^{\underline{b}}}$ that, from our present understanding, is the missing analog to the $4 \mathrm{D}, \mathcal{N}=1 G_{\underline{a}}$-tensor.

In the work of [21], an analysis based on prepotential superfields was undertaken regarding the scale compensating superfield $\Psi$ and conformal semi-prepotential $\hat{\mathrm{H}}_{\alpha}{ }^{a}$. It was shown these play an interesting role with respect to the emergence of Weyl scaling covariance in $11 \mathrm{D}, \mathcal{N}=1$ superspace. Three key points were noted in this context:

(a.) When a sufficiency of conventional constraints are imposed upon the 11D, $\mathcal{N}=1$ Poincaré superspace supergravity covariant derivative operators $\left(\nabla_{\alpha}, \nabla_{\underline{a}}\right)$ so that $\Psi$ and $\hat{\mathrm{H}}_{\alpha}$ a are the only independent superfields within them, a spinorial connection field $\mathcal{J}_{\alpha}{ }^{(+)}$(for Weyl scaling in superspace) emerges among the dimension one-half Poincaré supergravity supertensor components.

(b.) The sufficiency of conventional constraints that leads to the existence of $\mathcal{J}_{\alpha}{ }^{(+)}$is also sufficient to lead to the emergence of a vectorial connection field $\mathcal{J}_{\underline{a}}{ }^{(+)}$for Weyl scaling in superspace.

(c.) The existence of $\mathcal{J}_{\alpha}{ }^{(+)}$and $\mathcal{J}_{\underline{a}}{ }^{(+)}$together with the existence of the superspace supergravity covariant derivative operators $\left(\nabla_{\alpha}, \nabla_{\underline{a}}\right)$ imply the existence of modified supergravity covariant derivative operators $\left(\hat{\nabla}_{\alpha}, \hat{\nabla}_{\underline{a}}\right)$ that transform covariantly with respect to Weyl scaling.

In fact, precisely these three points are at the foundation of a paper written in 1991 within the context of $10 \mathrm{D}, \mathcal{N}=1$ superspace [7].

As the focus of this analysis is the modified supergravity covariant derivative operators (and their related field strengths, Bianchi identities, etc.), this approach is mute on implications for the superfields needed to construct the superframe superfields. This is true for most of the citations among [1]-[19] as they are not focused upon the superfield variables that are "inside" of $\left(\nabla_{\alpha}, \nabla_{\underline{a}}\right)$, i.e. the prepotentials.

We will make an observation about the relation of the $11 \mathrm{D}, \mathcal{N}=1$ Nordström theory and the infinitesimal super Weyl transformation laws of the complete non-linear Poincaré superspace supergravity derivatives $\left(\nabla_{\alpha}, \nabla_{\underline{a}}\right)$ as motivating a pathway for the derivation of similar results in all ten dimensional superspaces. Inspired by the success of relating the emergence of Weyl symmetry in $11 \mathrm{D}, \mathcal{N}=1$ superspace, together with our recent studies of supergravity in eleven and ten dimensions [22-25], we are motivated to extend the discussion and results of [21] into the domain of all ten dimensional supergravity theories 
in superspace. This is one purpose of this paper. However, we also wish to push beyond this boundary.

The structure of $4 \mathrm{D}, \mathcal{N}=2$ supergravity at the linearized level is very informative for any reader who is concerned about how the gauge degrees of the supergravity 3 -form can arise from the use of prepotentials. We will in the following use the work shown in [26] to discuss the mechanism seen for how this is to occur.

The relevance of this past work provided the first superspace description involving prepotentials where the supergravity supermultiplet contains (in the Poincaré limit) a gauge propagating physical degrees of freedom. In this case this field is a 1-form. It is analogous to the 3 -form in the $11 \mathrm{D}$ case.

The work of [26] showed that $4 \mathrm{D}, \mathcal{N}=2$ superfield supergravity required three different prepotentials:

(a.) a conformal Weyl prepotential,

(b.) a conformal compensating prepotential, and

(c.) an $\mathrm{SU}(2)$ compensating prepotential.

The last of these is required as there is an $\mathrm{SU}(2)$ subgroup of the superconformal group appropriate to this theory. As in 11D current indications are that there is no such similar subgroup and thus it seems a reasonable speculation a corresponding compensator is absent. On this basis, we have made the assumption that the eventual formulation of the 11D theory in a standard Salam-Strathdee superspace will not contain the third type of prepotential.

The work of [26] also reveals another fact. It was shown possible in that work to study the geometry of the superspace in a limit where only the Weyl prepotential is present. This is the limit under study in our efforts in both ten and eleven dimensional theories.

Many years ago, a then surprising discovery about the formulation of supergravity in superspace was revealed. The dynamics of the on-shell systems can be derived simply for using the superspace supergravity Bianchi and imposing a set of constraints on the torsion and curvature super tensors. This was first shown in the works of $[27,28]$. Our efforts are directed along this historical arc and do not introduce any assumption about augmenting the structure of Salam-Strathdee superspace such as assumptions in other formulations [29] (e.g. "pure spinors, etc.").

In an effort to be completely clear about this point, we wish to expand on the meaning we assign to the term "Salam-Strathdee superspace" to be contrasted with the term "Wess-Zumino superspace". We also want to make precise what we mean by additional assumptions. A superspace supergravity covariant derivative operator $\nabla_{A}=\left(\nabla_{\alpha}, \nabla_{\underline{a}}\right)$ will satisfy an equation of the form

$$
\left[\nabla_{\alpha}, \nabla_{\beta}\right]=T_{\alpha \beta}{ }^{\gamma} \nabla_{\gamma}+T_{\alpha \beta^{\underline{c}}} \nabla_{\underline{c}}+\frac{1}{2} R_{\alpha \beta \underline{c}^{\underline{d}}} \mathcal{M}_{\underline{\underline{d}}}^{\underline{\underline{c}}} .
$$

When we use the term "Salam-Strathdee" superspace theory of supergravity, we consider the additional assumption that

$$
\left(\gamma_{\underline{d}}\right)^{\alpha \beta} T_{\alpha \beta^{\underline{c}}} \propto \delta_{\underline{d}^{\underline{c}}}
$$


along other "conventional constraints" that algebraically relate one superfield to another. A general discussion of "conventional constraints" for supergravity can be seen in the works of $[4,6]$ and for the particular case of $11 \mathrm{D}$ in [34].

The constraint in (1.2) is a weaker constraint than

$$
T_{\alpha \beta^{\underline{c}}} \propto\left(\gamma^{\underline{c}}\right)_{\alpha \beta} .
$$

In this latter case we refer to this as a "Wess-Zumino superspace theory of supergravity". As discussed in the work of [29], in flat superspace both of these definitions coincide and by the introduction of a pure spinor superfield $\lambda^{\alpha}$ to multiply the spinorial supercovariant derivative $\nabla_{\alpha}$ one can form the product $\lambda^{\alpha} \nabla_{\alpha}$. This last operator is a BRST charge. However, with the assumption of (1.2), this product is not a BRST charge. Therefore, in this regard (1.3) makes additional assumptions over and above those in (1.2).

If a complete analysis without such added structures implied by (1.3) should fail, that would constitute a proof of the necessity of such structures. But to our knowledge no such complete analysis exists in the literature.

In our recent works of [23-25], we developed algorithmically based techniques that permit the investigation of the component field contents of possible supergravity (SG) prepotentials. This is a capacity that never before existed to our knowledge in the context of high dimensional superfield SG theories. Based on an assumption about the $11 \mathrm{D}, \mathcal{N}=1$ SG prepotential superfield, this allows us to identify candidate $10 \mathrm{D}, \mathcal{N}=1$ superfields that are most likely the $10 \mathrm{D}, \mathcal{N}=1 \mathrm{SG}$ prepotential superfield and four additional $10 \mathrm{D}$, $\mathcal{N}=1$ superfields that are the most likely candidates to describe the matter gravitino multiplet prepotential required to formulate Type-II theories. Identifying such candidate prepotentials is the major other purpose of this work.

In every superspace formulation where the prepotential formulation is completely understood, the supergravity prepotential transforms as the first superspace spinorial derivative of a parameter superfield ${ }^{1}$ that is consistent with the Lorentz structure of the supergravity prepotential and the presence of the superpace spinorial derivative. Thus, in a case where the supergravity prepotential transforms as a scalar under the associated Lorentz group, its gauge transformation parameter superfield is a spinor. Thus, we conjecture this sort of rule holds for the cases under discussion. This will be work for the future enabled by the ability to "scan" superfields as described in section five.

\section{Review of $11 \mathrm{D}, \mathcal{N}=1$ supergravity derivatives \& scale transforma- tions}

The spinorial 11D frame operator may be parametrized in the following equations, ${ }^{2}$

$$
\begin{aligned}
\mathrm{E}_{\alpha} & =\Psi^{1 / 2}\left[\exp \left(\frac{1}{2} \mathcal{A}^{\underline{a}} \underline{\underline{b}} \gamma_{\underline{a}} \underline{b}\right)\right] \alpha^{\beta}\left[\mathcal{N}_{\beta}{ }^{\gamma}\right]\left[\mathrm{D}_{\gamma}+\hat{\mathrm{H}}_{\gamma^{\underline{b}}} \partial_{\underline{b}}\right], \\
\mathcal{N}_{\alpha}{ }^{\beta} & \equiv\left[\mathbf{I}+\mathcal{A}^{\underline{a}} \gamma_{\underline{a}}+\frac{1}{3 !} \mathcal{A}^{[\underline{[}]} \gamma_{[\underline{3}]}+\frac{1}{4 !} \mathcal{A}^{[\underline{4}]} \gamma_{[4]}+\frac{1}{5 !} \mathcal{A}^{[5]} \gamma_{[\underline{5}]}\right] \alpha^{\beta},
\end{aligned}
$$

\footnotetext{
${ }^{1}$ However, such parameter superfields may themselves be expressed as spinor derivative operators of more fundamental parameter superfields.

${ }^{2}$ Roughly speaking, this is the superspace supergravity equivalent to the ADM [30-32] formulation but for the spinorial superframe.
} 
where $\Psi$ is the "scale compensator", $\mathcal{A}^{\underline{a}} \underline{b}$ is the "Lorentz compensator", $\mathcal{N}_{\alpha}^{\beta}$ is a "coset factor", and $\hat{\mathrm{H}}_{\beta} \underline{\underline{b}}$ is the "conformal graviton semi-prepotential". The parametrization of $\mathcal{N}_{\alpha}{ }^{\beta}$ shown in (2.1) is strongly dependent on the dimensionality of the superspace. For example, in simple supergravity theory in two dimensions, ${ }^{3}$ only the first two terms are present [33].

A slightly different, but equivalent parametrization was introduced in a previous work [34]. Without loss of generality, a slightly different semi-prepotential can be written in the form

$$
\hat{\mathrm{H}}_{\beta^{\underline{b}}}^{\underline{b}}=\mathrm{H}_{\beta^{\underline{b}}}-\frac{1}{\mathrm{D}}\left(\gamma^{\underline{\underline{b}}} \gamma_{\underline{d}}\right)_{\beta}{ }^{\delta} \mathrm{H}_{\delta^{\underline{d}}},
$$

where $\mathrm{D}=11$ for this eleven dimensional case. This also implies there is a local symmetry of the form

$$
\mathrm{H}_{\beta^{\underline{\underline{b}}}} \rightarrow \mathrm{H}_{\beta^{\underline{\underline{b}}}}+\left(\gamma^{\underline{\underline{b}}}\right)_{\beta}^{\alpha} \Lambda_{\alpha}
$$

that applies to (2.2).

All previous experience in superspace supergravity implies the vectorial 11D frame operator $\mathrm{E}_{\underline{a}}$, the spinorial spin connection $\omega_{\alpha \underline{c d}}$, and the vectorial spin connection $\omega_{\underline{a}} \underline{c d}$ must be determined in terms of the content of the spinorial 11D frame field. This is done by the imposition of "conventional constraints" on the torsion and curvature supertensors defined in (2.5). However, as first noted in the work [33], "conventional constraints" must also be imposed to determine $\mathcal{N}_{\alpha}^{\beta}$ solely in terms of $\mathrm{H}_{\beta} \underline{\underline{b}}$, but totally independent of $\Psi$. This has powerful implications for constraints in superfield supergravity.

A Poincaré supergeometry of the 11D theory demands the introduction of superspace supergravity covariant derivatives $\nabla_{\alpha}$ and $\nabla_{\underline{a}}$ defined by

$$
\begin{aligned}
& \nabla_{\alpha}=\mathrm{E}_{\alpha}+\frac{1}{2} \omega_{\alpha \underline{d}} \underline{e}^{\underline{e}} \mathcal{M}_{\underline{e}}^{\underline{d}}, \\
& \nabla_{\underline{a}}=\mathrm{E}_{\underline{a}}+\frac{1}{2} \omega_{\underline{a}} \underline{d}^{\underline{e}} \mathcal{M}_{\underline{\underline{e}}}^{\underline{d}} .
\end{aligned}
$$

Upon calculating the graded commutator of these leads to superspace torsions and curvatures according to,

$$
\begin{aligned}
& {\left[\nabla_{\alpha}, \nabla_{\beta}\right\}=T_{\alpha \beta} \underline{\underline{c}} \nabla_{\underline{c}}+T_{\alpha \beta}^{\gamma} \nabla_{\gamma}+\frac{1}{2} R_{\alpha \beta} \underline{d}^{\underline{e}} \mathcal{M}_{\underline{\underline{e}}}^{\underline{\underline{d}}},} \\
& {\left[\nabla_{\alpha}, \nabla_{\underline{b}}\right\}=T_{\alpha \underline{\underline{b}} \underline{\underline{c}}} \nabla_{\underline{c}}+T_{\alpha \underline{b}}^{\gamma} \nabla_{\gamma}+\frac{1}{2} R_{\alpha \underline{b}} \underline{d}^{\underline{e}} \mathcal{M}_{\underline{\underline{e}}}{ }^{\underline{d}},} \\
& {\left[\nabla_{\underline{a}}, \nabla_{\underline{b}}\right\}=T_{\underline{a} b}{ }^{\underline{c}} \nabla_{\underline{c}}+T_{\underline{a} b}^{\gamma} \nabla_{\gamma}+\frac{1}{2} R_{\underline{a} b \underline{\underline{d}}} \mathcal{M}_{\underline{\underline{e}}}^{\underline{d}} .}
\end{aligned}
$$

Based on an analysis of constraints for the 11D vielbein, the work in [21] concluded the set

$$
i \frac{1}{32}\left(\gamma_{\underline{a}}\right)^{\alpha \beta} T_{\alpha \beta^{\underline{b}}}=\delta_{\underline{\underline{a}}}^{\underline{b}}, \quad\left(\gamma_{\underline{a}}\right)^{\alpha \beta} T_{\alpha \beta}{ }^{\gamma}=0,
$$

\footnotetext{
${ }^{3}$ To our knowledge, the case of $\mathrm{D}=2$ is the only one in existence where the dependence [33] of $\mathcal{N}_{\alpha}{ }^{\beta}$ on $\mathrm{H}_{\alpha}{ }^{\underline{a}}$ has been explicitly presented. However, other complete solutions for constaints implicitly contain such information.
} 


$$
\begin{aligned}
& T_{\alpha[\underline{d e}]}-\frac{2}{55}\left(\gamma_{\underline{d e}}\right)_{\alpha}^{\gamma} T_{\gamma \underline{b}} \underline{b}=0, \\
& \left(\gamma_{\underline{a}}\right)^{\alpha \beta} R_{\alpha \beta} \underline{d e}=0, \\
& \left(\gamma_{\underline{a b c d e}}\right)^{\alpha \beta} T_{\alpha \beta^{\underline{e}}}=0, \\
& \left(\gamma_{[\underline{a b c d e} \mid}\right)^{\alpha \beta} T_{\alpha \beta \mid f]}=0, \\
& \left(\gamma_{\underline{a b}}\right)^{\alpha \beta} T_{\alpha \beta} \underline{b}=0, \\
& \left(\gamma_{[\underline{a b} \mid}\right)^{\alpha \beta} T_{\alpha \beta \mid c]}=0,
\end{aligned}
$$

can be enforced without implying any dynamical consequences on the components fields that remain with the vielbein. The first two constraints in (2.6) ensure that the graviton and gravitino, respectively, that appear at first order in the $\theta$-expansion of in $\nabla_{\alpha}$ are identified with the self-same fields that appear at zeroth order in $\nabla_{\underline{a}}$. The next two constraints have the same effects by removing the Lorentz connection of the theory (as an independent variable), and defining it in terms of the anholonomy associated with the component frame field. These second two constraints of (2.6) also ensure that the spin-connection that appears at first order in the $\theta$-expansion of in $\nabla_{\alpha}$ is identified with the self-same field appearing at zeroth order in $\nabla_{\underline{a}}$. The last four constraints in (2.6) remove all the superfields that appear in $\mathcal{N}_{\alpha}{ }^{\beta}$ as independent quantities and makes them non-trivially dependent only on $\mathrm{H}_{\alpha}{ }^{a}$. It is directly possible to prove at the linearized order. However, beyond this order the process is expected to be horribly non-linear as in the $2 \mathrm{D}, \mathcal{N}=1$ supergravity theory.

Recent calculations [24] inspired by adinkras have yielded IT-based techniques providing unprecedented access to the component field composition of superfields in 11D. Based on this, we have proposed the set of constraints above may be strengthened by the replacement as indicated below,

$$
\begin{aligned}
& i \frac{1}{32}\left(\gamma_{\underline{a}}\right)^{\alpha \beta} T_{\alpha \beta^{\underline{b}}}=\delta_{\underline{a}}^{\underline{b}}, \quad\left(\gamma_{\underline{a}}\right)^{\alpha \beta} T_{\alpha \beta}{ }^{\gamma}=0, \\
& T_{\alpha[\underline{d e}]}-\frac{2}{55}\left(\gamma_{\underline{d e}}\right)_{\alpha}{ }^{\gamma} T_{\gamma \underline{\underline{b}}}=0, \quad\left(\gamma_{\underline{a}}\right)^{\alpha \beta} R_{\alpha \beta} \underline{\underline{d e}}=0 \text {, } \\
& \left(\gamma_{\underline{a b c d e}}\right)^{\alpha \beta} T_{\alpha \beta^{\underline{e}}}=0, \quad\left(\gamma_{[\underline{a b c d e \mid}}\right)^{\alpha \beta} T_{\alpha \beta \mid f]}=0, \\
& \left(\gamma_{\underline{a b}}\right)^{\alpha \beta} T_{\alpha \beta^{\underline{c}}}=0,
\end{aligned}
$$

as this set, "CGNN constraints" [12], is consistent with the choice of a scalar superfield $\mathcal{V}$ as the supergravity prepotential. This implies the superfunction $\mathrm{H}_{\beta} \underline{\underline{c}}=\mathrm{H}_{\beta} \underline{c}(\mathcal{V})$ must admit a functional dependence that satisfies this equation. This functional dependence must involve fifteen powers of $\mathrm{D}_{\alpha}$ acting on $\mathcal{V}$.

We highlight the following two points which support the complete vanishing of the final constraint shown in (2.7) in the limit where only the proposed scalar supergravity prepotential is retained:

(a.) a complete parametrization of all possible degrees of freedom of the 11D spinorial supervielbein was given by (2.1) in our paper. The constraints shown in (2.6) determine all of the $\mathcal{A}$-superfields within $\mathcal{N}_{\alpha}{ }^{\beta}$ in terms of the $\hat{H}$ superfield based on linearized calculations reported in the work of [34].

(b.) the $\hat{H}$-superfield is a "semi-prepotential" along the lines of such superfields discussed in the work of [26]. In turn, however, our work of [23] using our "scanning" techniques 
showed that the representation required for the final line of (2.7) to be non-zero is absent from the proposed scalar supergavity prepotential. Thus, we conclude that torsion must vanish.

We invite any skeptic unfamiliar with the workings of supergravity prepotential formulations to undertake the proof this final constraint to show the accuracy of our statements.

A solution to (2.7) is provided by making the "shift" $\Psi \rightarrow 1+\Psi$ (equations (12) and (18) in [21]) so that

$$
\begin{aligned}
\nabla_{\alpha}= & \mathrm{D}_{\alpha}+\frac{1}{2} \Psi \mathrm{D}_{\alpha}+\frac{1}{10}\left(\mathrm{D}_{\beta} \Psi\right)(\gamma \underline{\underline{d e}})_{\alpha}^{\beta} \mathcal{M}_{\underline{d e}}, \\
\nabla_{\underline{a}}= & \partial_{\underline{a}}+\Psi \partial_{\underline{a}}+i \frac{1}{4}\left(\gamma_{\underline{a}}\right)^{\alpha \beta}\left(\mathrm{D}_{\alpha} \Psi\right) \mathrm{D}_{\beta}+\frac{1}{5}\left(\partial_{\underline{c}} \Psi\right) \mathcal{M}_{\underline{a}} \underline{\underline{c}} \\
& +i \frac{1}{160}\left(\gamma_{\underline{a}} \underline{d e}\right)^{\alpha \beta}\left(\mathrm{D}_{\alpha} \mathrm{D}_{\beta} \Psi\right) \mathcal{M}_{\underline{d e}},
\end{aligned}
$$

where $\Psi$ in (2.8) is an infinitesimal superfield and we set the superfield $\mathrm{H}_{\beta} \underline{\underline{c}}=0$. It should also be noted we can regard the supervector fields here as describing a Nordström supergravity theory as in our previous work [22], although we used a different set of constraints in $[22]$.

In turn, (2.8) suggests the definition of a set of Weyl scaling transformations of the full superspace covariant derivatives given by

$$
\begin{aligned}
\delta_{S} \nabla_{\alpha}= & \frac{1}{2} L \nabla_{\alpha}+\frac{1}{10}\left(\nabla_{\beta} L\right)\left(\gamma^{\underline{d e}}\right)_{\alpha}^{\beta} \mathcal{M}_{\underline{d e}}, \\
\delta_{S} \nabla_{\underline{a}}= & L \nabla_{\underline{a}}+i \frac{1}{4}\left(\gamma_{\underline{a}}\right)^{\alpha \beta}\left(\nabla_{\alpha} L\right) \nabla_{\beta}+\frac{1}{5}\left(\nabla_{\underline{c}} L\right) \mathcal{M}_{\underline{a}} \underline{\underline{c}} \\
& +i \frac{1}{160}\left(\gamma_{\underline{a}} \underline{d e}\right)^{\alpha \beta}\left(\nabla_{\alpha} \nabla_{\beta} L\right) \mathcal{M}_{\underline{d e}} .
\end{aligned}
$$

In the results shown in (2.9), the superspace covariant derivatives $\nabla_{\alpha}$ and $\nabla_{\underline{a}}$ are "full" superspace covariant derivatives where the only remaining independent superfield variables are $\Psi$ and $\mathrm{H}_{\beta} \underline{c}(\mathcal{V})$.

It should be noted there is a lesson to learn from (2.8) and (2.9). A set of full Weyl scale transformation laws on the superspace supergravity supercovariant derivative operators can be obtained by starting from the formulation of a Nordström superspace supergravity theory, acting with a superspace scaling operation $\delta_{S}$ on the Nordström scalar field in the theory, calling $\delta_{S} \Psi=L$, and finally replaces all "bare" derivative operators by full superspace supergravity supercovariant derivative operators.

In turn this implies we are able to analyze the Weyl scaling properties of all the superspace torsion and curvature supertensors with engineering dimensions of less than three-halves to find,

$$
\begin{aligned}
\delta_{S} T_{\alpha \beta^{\underline{c}}}= & 0, \\
\delta_{S} T_{\alpha \beta}{ }^{\gamma}= & \left.\frac{1}{2} L T_{\alpha \beta}{ }^{\gamma}-i \frac{1}{4} T_{\alpha \beta^{\underline{c}}}\left(\gamma_{\underline{c}}\right)^{\delta \gamma}\left(\nabla_{\delta} L\right)+\frac{1}{2}\left(\nabla_{(\alpha} L\right) \delta_{\beta}\right)^{\gamma} \\
& +\frac{1}{20}\left(\nabla_{\delta} L\right)\left(\gamma^{[2]}\right)_{(\alpha}{ }^{\delta}\left(\gamma_{[2]}\right)_{\beta)}{ }^{\gamma},
\end{aligned}
$$




$$
\begin{aligned}
& \delta_{S} T_{\alpha \underline{b}} \underline{\underline{c}}=\frac{1}{2} L T_{\alpha \underline{b}} \underline{\underline{c}}-i \frac{1}{4}\left(\gamma_{\underline{b}}\right)^{\gamma \delta}\left(\nabla_{\gamma} L\right) T_{\alpha \delta^{\underline{c}}}+\left(\nabla_{\alpha} L\right) \delta_{\underline{b}} \underline{c} \\
& +\frac{1}{5}\left(\nabla_{\gamma} L\right)\left(\gamma_{\underline{b}}^{\underline{c}}\right)_{\alpha}^{\gamma} \text {, } \\
& \delta_{S} T_{\alpha \underline{b}}^{\gamma}=L T_{\alpha \underline{b}}^{\gamma}-i \frac{1}{4}\left(\gamma_{\underline{b}}\right)^{\delta \epsilon}\left(\nabla_{\delta} L\right) T_{\alpha \epsilon}^{\gamma}-i \frac{1}{4} T_{\alpha \underline{\underline{b}}}{ }^{\underline{c}}\left(\gamma_{\underline{c}}\right)^{\delta \gamma}\left(\nabla_{\delta} L\right) \\
& -\frac{1}{2}\left(\nabla_{\underline{b}} L\right) \delta_{\alpha}^{\gamma}-\frac{1}{10}\left(\nabla_{\underline{d}} L\right)\left(\gamma_{\underline{b}}\right)_{\alpha}^{\gamma} \\
& +i \frac{1}{4}\left(\gamma_{\underline{b}}\right)^{\delta \gamma}\left(\nabla_{\alpha} \nabla_{\delta} L\right)-i \frac{1}{320}\left(\gamma_{\underline{b}} \underline{d e}\right)^{\delta \epsilon}\left(\nabla_{\delta} \nabla_{\epsilon} L\right)\left(\gamma_{\underline{d e}}\right)_{\alpha}^{\gamma} \text {, }
\end{aligned}
$$

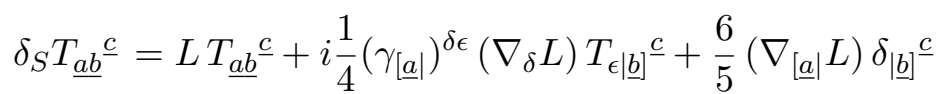

$$
\begin{aligned}
& +i \frac{1}{40}\left(\gamma_{\underline{a b}}^{\underline{c}}\right)^{\delta \epsilon}\left(\nabla_{\delta} \nabla_{\epsilon} L\right) \text {, } \\
& \left.\delta_{S} R_{\alpha \beta} \underline{\underline{d e}}=L R_{\alpha \beta} \underline{\underline{d e}}+\frac{1}{5} T_{\alpha \beta} \underline{[d}\left(\nabla^{e}\right] L\right)+\frac{1}{5} T_{\alpha \beta} \delta\left(\nabla_{\gamma} L\right)\left(\gamma^{\underline{d e}}\right)_{\delta}^{\gamma} \\
& -\frac{1}{5}\left(\nabla_{(\alpha \mid} \nabla_{\gamma} L\right)\left(\gamma^{\underline{d e}}\right)_{\mid \beta)}{ }^{\gamma}+i \frac{1}{80} T_{\alpha \beta^{\underline{c}}}\left(\gamma_{\underline{c}} \underline{d e}\right)^{\delta \epsilon}\left(\nabla_{\delta} \nabla_{\epsilon} L\right) .
\end{aligned}
$$

The constraints on the last three lines of (2.7) have a solution given by [12] ${ }^{4}$

$$
T_{\alpha \beta^{\underline{c}}}=i\left(\gamma^{\underline{c}}\right)_{\alpha \beta}+i \frac{1}{32 \cdot 5 !}\left(\gamma^{\gamma^{1} \cdots \underline{b_{5}}}\right)_{\alpha \beta} X_{\underline{b}_{1} \cdots \underline{b}_{5}}, X_{\underline{a b c d e^{e}}}=X_{[\underline{a b c d e} \underline{f}]}=0
$$

where $X_{\underline{a}_{1} \ldots \underline{a}_{5}} \underline{\underline{b}} \equiv i\left(\gamma_{\underline{a}_{1} \cdots \underline{a}_{5}}\right)^{\alpha \beta} T_{\alpha \beta^{\underline{b}}}$ alternatively. ${ }^{5}$ The expression of $T_{\alpha \beta} \underline{\underline{c}}$ may be substituted in (2.11) and (2.12). Contraction of the $\beta$ and $\gamma$ indices in the former and the $\underline{b}$ and $\underline{c}$ indices in the latter yields respectively

$$
\begin{aligned}
& \delta_{S} T_{\alpha \beta}{ }^{\beta}=\frac{1}{2} L T_{\alpha \beta}^{\beta}-i \frac{1}{4}\left[i\left(\gamma^{\underline{c}}\right)_{\alpha \beta}+i \frac{1}{32 \cdot 5 !}\left(\gamma^{\underline{b_{1}} \cdots \underline{b}_{5}}\right)_{\alpha \beta} X_{\underline{b}_{1} \cdots \underline{b}_{5}} \underline{\underline{c}}\right]\left(\gamma_{\underline{c}}\right)^{\delta \beta}\left(\nabla_{\delta} L\right) \\
& \left.+\frac{1}{2}\left(\nabla_{(\alpha} L\right) \delta_{\beta}{ }^{\beta}+\frac{1}{20}\left(\nabla_{\delta} L\right)\left(\gamma^{[2]}\right)_{(\alpha}{ }^{\delta}\left(\gamma_{[2]}\right)_{\beta}\right)^{\beta} \\
& \left.=\frac{1}{2} L T_{\alpha \beta}^{\beta}+\frac{1}{4}\left(\gamma^{\underline{c}}\right)_{\alpha \beta}\left(\gamma_{\underline{c}}\right)^{\delta \beta}\left(\nabla_{\delta} L\right)+\frac{1}{2}\left(\nabla_{(\alpha} L\right) \delta_{\beta}\right)^{\beta} \\
& \left.+\frac{1}{20}\left(\nabla_{\delta} L\right)\left(\gamma^{[2]}\right)_{(\alpha}^{\delta}\left(\gamma_{[2]}\right)_{\beta}\right)^{\beta} \\
& =\frac{1}{2} L T_{\alpha \beta}{ }^{\beta}+\frac{33}{4}\left(\nabla_{\alpha} L\right) \text {, } \\
& \delta_{S} T_{\alpha \underline{b}} \underline{\underline{b}}=\frac{1}{2} L T_{\alpha \underline{b}}^{\underline{b}}+\frac{1}{4}\left(\gamma_{\underline{b}}\right)^{\gamma \delta}\left(\nabla_{\gamma} L\right)\left[\left(\gamma^{\underline{b}}\right)_{\alpha \delta}+\frac{1}{32 \cdot 5 !}\left(\gamma^{\underline{b_{1}} \cdots \underline{b}_{5}}\right)_{\alpha \delta} X_{\underline{b}_{1} \cdots \underline{b}_{5}}\right]+\left(\nabla_{\alpha} L\right) \delta_{\underline{b}} \underline{\underline{b}} \\
& =\frac{1}{2} L T_{\alpha \underline{b}} \underline{b}+\frac{1}{4}\left(\gamma_{\underline{b}}\right)^{\gamma \delta}\left(\nabla_{\gamma} L\right)\left(\gamma^{\underline{b}}\right)_{\alpha \delta}+\left(\nabla_{\alpha} L\right) \delta_{\underline{b}} \underline{b} \\
& =\frac{1}{2} L T_{\alpha \underline{\underline{b}}}+\frac{33}{4}\left(\nabla_{\alpha} L\right) \text {. }
\end{aligned}
$$

\footnotetext{
${ }^{4}$ The first indication of the need for modification to the superspace torsion proportional to $\gamma / \sigma$ matrices was noted in 1983 [39] in the context of $4 \mathrm{D}, \mathcal{N}>4$ conformal supergravity in superspace.

${ }^{5}$ Notice that the normalization of the $X_{[5]} \underline{b}$ differs by a factor of 32 with that in the work of [21], i.e. the factor $\frac{1}{32}$ is put in equation (2.16) instead of the definition of $X_{[5]} \underline{b}$.
} 
The equation in the final portion of the results shown in (2.16) is responsible for the disappearance of the $X$-tensors from the second lines in (2.17) and (2.18).

If we make the definitions

$$
\mathcal{J}_{\alpha}{ }^{(1)}=\frac{4}{33} T_{\alpha \beta}{ }^{\beta}, \mathcal{J}_{\alpha}{ }^{(2)}=\frac{4}{33} T_{\alpha \underline{\underline{b}}}{ }^{\underline{b}},
$$

then we see

$$
\delta_{S} \mathcal{J}_{\alpha}^{(1)}=\frac{1}{2} L \mathcal{J}_{\alpha}{ }^{(1)}+\left(\nabla_{\alpha} L\right), \delta_{S} \mathcal{J}_{\alpha}{ }^{(2)}=\frac{1}{2} L \mathcal{J}_{\alpha}{ }^{(2)}+\left(\nabla_{\alpha} L\right) .
$$

Upon changing basis for these superfields by defining

we observe that

$$
\mathcal{J}_{\alpha}{ }^{( \pm)}=\frac{1}{2}\left[\mathcal{J}_{\alpha}{ }^{(1)} \pm \mathcal{J}_{\alpha}{ }^{(2)}\right]
$$

$$
\delta_{S} \mathcal{J}_{\alpha}^{(+)}=\frac{1}{2} L \mathcal{J}_{\alpha}{ }^{(+)}+\left(\nabla_{\alpha} L\right), \delta_{S} \mathcal{J}_{\alpha}^{(-)}=\frac{1}{2} L \mathcal{J}_{\alpha}{ }^{(-)} .
$$

While $\mathcal{J}_{\alpha}{ }^{(-)}$transforms like a scale covariant tensor of weight $\frac{1}{2}$, the quantity $\mathcal{J}_{\alpha}{ }^{(+)}$ transforms with a scale weight of $\frac{1}{2}$ while being a spinorial gauge connection under a scaling transformation! The work of [20] indicated that such a dimension one-half supertorsion tensor was required for an off-shell description of $11 \mathrm{D}, \mathcal{N}=1$ Poincaré supergravity.

According to the analysis in [21], there is also necessarily an engineering dimension one superfield $\mathcal{W}_{\underline{a b c d}}$ with Weyl weight one (i.e. $\delta_{S} \mathcal{W}_{\underline{a b c d}}=L \mathcal{W}_{\underline{a b c d}}$ ) defined by ${ }^{6}$

$$
\mathcal{W}_{\underline{a b c d}} \equiv \frac{1}{32}\left[\left(\gamma^{\underline{\underline{e}}} \gamma_{\underline{a b c d}}\right)_{\gamma}^{\alpha} T_{\alpha \underline{\underline{e}}}^{\gamma}+i \frac{11}{4}\left(\gamma_{\underline{a b c d}}\right)^{\alpha \beta}\left(\nabla_{\alpha} \mathcal{J}_{\beta}^{(+)}-\frac{23}{220} \mathcal{J}_{\alpha}^{(+)} \mathcal{J}_{\beta}^{(+)}\right)\right]
$$

and which contains all the on-shell degrees of freedom. In particular, the lowest order term in this superfield (i.e. setting $\theta=0$ ) is the supercovariantized field strength of the component level 3-form gauge field. Moreover, the 11D supercovarinatized Weyl-gravitino field strength is contained at the first order in the $\theta$-expansion, and the supercovariantized Weyl tensor of the 11D bosonic spacetime is contained at the second order in the $\theta$-expansion of $\mathcal{W}_{\underline{a b c d}}$.

Thus, we conclude the most likely path forward for an off-shell, in the sense of the pioneering work in [27], $11 \mathrm{D}, \mathcal{N}=1$ superspace supergravity theory must be the construction, as we suspected in 1996 , on the basis of three superfields now known to be $\mathcal{W}_{\underline{a b c d}}, X_{\underline{a}_{1} \cdots \underline{a}_{5}} \underline{\underline{b}}$, and $\mathcal{J}_{\alpha}$. The ultimate deepest reason to expect this decomposition is the structure of gravity itself. When one considers the Riemann Tensor of ordinary gravity (which must be embedded within any supergravity theory), it can be decomposed into the:

(a.) Weyl,

(b.) Ricci, and

(c.) Scalar Curvature

portions. In our opinion, any superspace construction that ignores this tripartite division is unlikely to be adequate.

\footnotetext{
${ }^{6}$ Notice that the Weyl tensor $\mathcal{W}_{\underline{a b c d}}$ here differs by an overall factor of $i$ with that in the work of [21]. It is because in our convention here, all $\gamma$-matrices are real, while in $[21],\left(\gamma^{[1]}\right)_{\alpha \beta}$ is real but $\left(\gamma^{[4]}\right)_{\alpha \beta}$ is imaginary. Thus $\mathcal{W}_{\underline{a b c d}}$ is real in both cases.
} 


\section{Supergravity derivatives \& scale transformations in 10D}

The content of the previous section about the superspace supergravity supercovariant derivatives $\nabla_{\underline{A}}=\left(\nabla_{\alpha}, \nabla_{\underline{a}}\right)$, the torsion supertensors $T_{\underline{A}} \underline{B}$, and curvature supertensors $R_{\underline{A}} \underline{B} \underline{c}^{\underline{d}}$ can be directly used to derive similar results for the superspaces associated with Type-IIA and Type-I superstring theories. However, there is much more efficient and rapid way in this can be done. This alternative route is also directly applicable to the Type-IIB case. So rather than going the route of the dimensional reduction of these geometrical quantities, we choose to take a less obvious pathway.

The bottom line message from the last section is that the scalar compensator, which appears in the parametrization of the framefield (2.1), is isomorphic to the concept of a scalar theory (i.e. Nordström gravitation). This means explorations [22] of Nordström supergravity are equivalent to the exploration of the limit of a full superframe where only the compensator is retained. This has the implication that investigations of Nordström supergravity theories fix the dependences of the superspace supergravity supercovariant derivatives $\nabla_{\underline{A}}$, the torsion supertensors $T_{\underline{A}} \underline{B} \underline{C}$ and curvature supertensors $R_{\underline{A}} \underline{B} \underline{\underline{c}} \underline{\underline{d}}$ on the scale compensating superfield.

The constraints used in the following results can be derived from equations found in the work of [22]. Take the following indicated equations from that work which are linear in the first derivative of the scale compensator, use appropriate algebraic operations to express the first derivatives in terms of torsion, and substitute those expressions back into the equations. For the $\mathcal{N}=$ IIA supergeometry, the appropriate equations from which to start the derivation are given in (6.10)-(6.19), (6.22), and (6.25). For the $\mathcal{N}=$ IIB supergeometry, the appropriate equations from which to start the derivation are given in (7.11)-(7.20), (7.23), and (7.26). Finally, for the $\mathcal{N}=$ I supergeometry, the appropriate equations from which to start the derivation are given in (5.8), (5.9), (5.10), and (5.12). These are found to be equivalent to results given previously in [7].

\section{1 $10 \mathrm{D}, \mathcal{N}=$ IIA supergravity derivatives \& scale transformations}

The covariant derivatives (equations (8.51)-(8.53) in [22]) linear in the real conformal compensator $\Psi$ are given by

$$
\begin{aligned}
& \nabla_{\alpha}=\mathrm{D}_{\alpha}+\frac{1}{2} \Psi \mathrm{D}_{\alpha}+\frac{1}{10}\left(\sigma^{\underline{a} b}\right)_{\alpha}^{\beta}\left(\mathrm{D}_{\beta} \Psi\right) \mathcal{M}_{\underline{a} b}, \\
& \nabla_{\dot{\alpha}}=\mathrm{D}_{\dot{\alpha}}+\frac{1}{2} \Psi \mathrm{D}_{\dot{\alpha}}+\frac{1}{10}\left(\sigma^{\underline{a} b}\right)_{\dot{\alpha}}^{\dot{\beta}}\left(\mathrm{D}_{\dot{\beta}} \Psi\right) \mathcal{M}_{\underline{a} b}, \\
& \nabla_{\underline{a}}=\partial_{\underline{a}}+\Psi \partial_{\underline{a}}-i \frac{1}{5}\left(\sigma_{\underline{a}}\right)^{\delta \gamma}\left(\mathrm{D}_{\delta} \Psi\right) \mathrm{D}_{\gamma}-i \frac{1}{5}\left(\sigma_{\underline{a}}\right)^{\dot{\delta} \dot{\gamma}}\left(\mathrm{D}_{\dot{\delta}} \Psi\right) \mathrm{D}_{\dot{\gamma}}-\left(\partial_{\underline{c}} \Psi\right) \mathcal{M}_{\underline{a}}^{\underline{c}},
\end{aligned}
$$

where the "bare" algebra of the supersymmetry covariant derivatives and the spacetime partial derivative takes the forms

$$
\left\{\mathrm{D}_{\alpha}, \mathrm{D}_{\beta}\right\}=i\left(\sigma^{\underline{a}}\right)_{\alpha \beta} \partial_{\underline{a}},\left\{\mathrm{D}_{\dot{\alpha}}, \mathrm{D}_{\dot{\beta}}\right\}=i\left(\sigma^{\underline{a}}\right)_{\dot{\alpha} \dot{\beta}} \partial_{\underline{a}},\left\{\mathrm{D}_{\alpha}, \mathrm{D}_{\dot{\beta}}\right\}=0,
$$

which indicate the non-vanishing values of the torsion supertensors must be given by

$$
T_{\alpha{ }^{\beta}} \stackrel{c}{=}=i\left(\sigma^{\underline{c}}\right)_{\alpha \beta}, T_{\dot{\alpha} \dot{\beta}}^{\underline{c}}=i\left(\sigma^{\underline{c}}\right)_{\dot{\alpha} \dot{\beta}}, T_{\alpha \dot{\beta}} \underline{\underline{c}}=0,
$$

and noting $\left[\partial_{\underline{a}}, \partial_{\underline{b}}\right]=0$. 
Mimicking results shown in going from (2.8) to (2.9), we conclude the super scale transformation laws for the full superspace supercovariant supergravity derivative operators must take the forms which follow from the results in (3.1), (3.2), and (3.3). Thus, we write

$$
\begin{aligned}
& \delta_{S} \nabla_{\alpha}=\frac{1}{2} L \nabla_{\alpha}+\frac{1}{10}\left(\sigma^{\underline{a} b}\right)_{\alpha}^{\beta}\left(\nabla_{\beta} L\right) \mathcal{M}_{\underline{a} b}, \\
& \delta_{S} \nabla_{\dot{\alpha}}=\frac{1}{2} L \nabla_{\dot{\alpha}}+\frac{1}{10}\left(\sigma^{\underline{a} b}\right)_{\dot{\alpha}}^{\dot{\beta}}\left(\nabla_{\dot{\beta}} L\right) \mathcal{M}_{\underline{a} b}, \\
& \delta_{S} \nabla_{\underline{a}}=L \nabla_{\underline{a}}-i \frac{1}{5}\left(\sigma_{\underline{a}}\right)^{\delta \gamma}\left(\nabla_{\delta} L\right) \nabla_{\gamma}-i \frac{1}{5}\left(\sigma_{\underline{a}}\right)^{\dot{\delta} \dot{\gamma}}\left(\nabla_{\dot{\delta}} L\right) \nabla_{\dot{\gamma}}-\left(\nabla_{\underline{c}} L\right) \mathcal{M}_{\underline{a}} \underline{\underline{c}},
\end{aligned}
$$

as the super Weyl transformation laws of superspace supergravity derivatives for 10D, $\mathcal{N}=$ IIA theories.

Upon using the definitions of torsion and curvature tensors

$$
\begin{aligned}
& {\left[\nabla_{\alpha}, \nabla_{\beta}\right\}=T_{\alpha \beta} \underline{\underline{c}} \nabla_{\underline{c}}+T_{\alpha \beta}{ }^{\gamma} \nabla_{\gamma}+T_{\alpha \beta}{ }^{\dot{\gamma}} \nabla_{\dot{\gamma}}+\frac{1}{2} R_{\alpha \beta} \underline{d}^{\underline{e}} \mathcal{M}_{\underline{\underline{e}}}^{\underline{d}},} \\
& {\left[\nabla_{\alpha}, \nabla_{\dot{\beta}}\right\}=T_{\alpha \dot{\beta}} \underline{\underline{c}} \nabla_{\underline{c}}+T_{\alpha \dot{\beta}}^{\gamma} \nabla_{\gamma}+T_{\alpha \dot{\beta}}^{\dot{\gamma}} \nabla_{\dot{\gamma}}+\frac{1}{2} R_{\alpha \dot{\beta} \underline{\underline{d}}} \underline{\underline{e}} \mathcal{M}_{\underline{\underline{e}}}^{\underline{\underline{d}}},} \\
& {\left[\nabla_{\dot{\alpha}}, \nabla_{\dot{\beta}}\right\}=T_{\dot{\alpha} \dot{\beta}} \underline{c}^{\underline{c}} \nabla_{\underline{c}}+T_{\dot{\alpha} \dot{\beta}}{ }^{\gamma} \nabla_{\gamma}+T_{\dot{\alpha} \dot{\beta}}{ }^{\dot{\gamma}} \nabla_{\dot{\gamma}}+\frac{1}{2} R_{\dot{\alpha} \dot{\beta}} \underline{\underline{d}}^{\underline{e}} \mathcal{M}_{\underline{\underline{e}}}^{\underline{\underline{d}}},} \\
& {\left[\nabla_{\alpha}, \nabla_{\underline{b}}\right\}=T_{\alpha \underline{b}} \nabla_{\underline{c}}+T_{\alpha \underline{b}}{ }^{\gamma} \nabla_{\gamma}+T_{\alpha \underline{b}} \nabla_{\dot{\gamma}}^{\dot{\gamma}}+\frac{1}{2} R_{\alpha \underline{\underline{b}} \underline{d}^{\underline{e}}} \mathcal{M}_{\underline{\underline{e}}}^{\underline{d}},} \\
& {\left[\nabla_{\dot{\alpha}}, \nabla_{\underline{b}}\right\}=T_{\dot{\alpha} \underline{b}} \underline{c}_{\underline{c}} \nabla_{\underline{c}}+T_{\dot{\alpha} \underline{b}}^{\gamma} \nabla_{\gamma}+T_{\dot{\alpha} \underline{b}}{ }^{\dot{\gamma}} \nabla_{\dot{\gamma}}+\frac{1}{2} R_{\dot{\alpha} \underline{b}} \underline{d}^{\underline{e}} \mathcal{M}_{\underline{e}}^{\underline{d}},} \\
& {\left[\nabla_{\underline{a}}, \nabla_{\underline{b}}\right\}=T_{\underline{a} b}{ }^{\underline{c}} \nabla_{\underline{c}}+T_{\underline{a} b}{ }^{\gamma} \nabla_{\gamma}+T_{\underline{a b}}{ }^{\dot{\gamma}} \nabla_{\dot{\gamma}}+\frac{1}{2} R_{\underline{a} b \underline{d^{\underline{e}}}} \mathcal{M}_{\underline{\underline{e}}}^{\underline{d}},}
\end{aligned}
$$

appropriate to the case of the $10,=$ NIIA theory, we find the Weyl scaling properties of all the superspace torsion and curvature supertensors with weights of less than three-halves as below. Weyl scaling properties of all other superspace torsion and curvature supertensors with weights of equal and larger than three-halves will be presented in appendix A.1. Grouping according to scale. We find,

$$
\begin{aligned}
& \delta_{S} T_{\alpha \beta^{\underline{c}}}=0, \\
& \delta_{S} T_{\alpha \dot{\beta}} \stackrel{c}{=}=0, \\
& \delta_{S} T_{\dot{\alpha} \dot{\beta}} \stackrel{c}{=}=0 \text {, } \\
& \left.\left.\delta_{S} T_{\alpha \beta}{ }^{\gamma}=\frac{1}{2} L T_{\alpha \beta}{ }^{\gamma}+i \frac{1}{5} T_{\alpha \beta^{\underline{c}}}\left(\sigma_{\underline{c}}\right)^{\gamma \delta}\left(\nabla_{\delta} L\right)+\frac{1}{2}\left(\nabla_{(\alpha} L\right) \delta_{\beta}\right)^{\gamma}+\frac{1}{20}\left(\sigma^{[2]}\right)_{(\alpha}{ }^{\delta}\left(\sigma_{[2]}\right)_{\beta}\right)^{\gamma}\left(\nabla_{\delta} L\right) \text {, } \\
& \delta_{S} T_{\alpha \beta} \dot{\gamma}=\frac{1}{2} L T_{\alpha \beta} \dot{\gamma}+i \frac{1}{5} T_{\alpha \beta} \underline{\underline{c}}\left(\sigma_{\underline{c}}\right)^{\dot{\delta}}\left(\nabla_{\dot{\delta}} L\right), \\
& \left.\delta_{S} T_{\dot{\alpha} \dot{\beta}} \dot{\gamma}=\frac{1}{2} L T_{\dot{\alpha} \dot{\beta}} \dot{\gamma}+i \frac{1}{5} T_{\dot{\alpha} \dot{\beta}}{ }^{c}\left(\sigma_{\underline{c}}\right)^{\dot{\gamma} \dot{\delta}}\left(\nabla_{\dot{\delta}} L\right)+\frac{1}{2}\left(\nabla_{(\dot{\alpha}} L\right) \delta_{\dot{\beta}}\right)^{\dot{\gamma}}+\frac{1}{20}\left(\sigma^{[2]}\right)_{(\dot{\alpha}}^{\dot{\delta}}\left(\sigma_{[2]}\right)_{\dot{\beta})} \dot{\gamma}\left(\nabla_{\dot{\delta}} L\right) \text {, } \\
& \delta_{S} T_{\dot{\alpha} \dot{\beta}}^{\gamma}=\frac{1}{2} L T_{\dot{\alpha} \dot{\beta}}^{\gamma}+i \frac{1}{5} T_{\dot{\alpha} \dot{\beta}}^{\underline{c}}\left(\sigma_{\underline{c}}\right)^{\gamma \delta}\left(\nabla_{\delta} L\right)
\end{aligned}
$$




$$
\begin{aligned}
& \delta_{S} T_{\alpha \dot{\beta}}^{\gamma}=\frac{1}{2} L T_{\alpha \dot{\beta}}^{\gamma}+i \frac{1}{5} T_{\alpha \dot{\beta}} \underline{\underline{c}}\left(\sigma_{\underline{c}}\right)^{\delta \gamma}\left(\nabla_{\delta} L\right)+\frac{1}{2}\left(\nabla_{\dot{\beta}} L\right) \delta_{\alpha}^{\gamma}+\frac{1}{20}\left(\sigma^{[2]}\right)_{\dot{\beta}}^{\dot{\delta}}\left(\sigma_{[2]}\right)_{\alpha}^{\gamma}\left(\nabla_{\dot{\delta}} L\right), \\
& \delta_{S} T_{\alpha \dot{\beta}} \dot{\gamma}=\frac{1}{2} L T_{\alpha \dot{\beta}}^{\dot{\gamma}}+i \frac{1}{5} T_{\alpha \dot{\beta}} \underline{c}\left(\sigma_{\underline{c}}\right)^{\dot{\delta} \dot{\gamma}}\left(\nabla_{\dot{\delta}} L\right)+\frac{1}{2}\left(\nabla_{\alpha} L\right) \delta_{\dot{\beta}} \dot{\gamma}+\frac{1}{20}\left(\sigma^{[2]}\right)_{\dot{\beta}}^{\dot{\gamma}}\left(\sigma_{[2]}\right)_{\alpha} \delta\left(\nabla_{\delta} L\right) \text {, } \\
& \delta_{S} T_{\alpha \underline{\underline{b}}} \underline{\underline{c}}=\frac{1}{2} L T_{\alpha \underline{\underline{c}}} \underline{\underline{c}}+\left(\nabla_{\alpha} L\right) \delta_{\underline{\underline{b}}}^{\underline{c}}+\frac{1}{5}\left(\sigma_{\underline{\underline{b}}}^{\underline{\underline{c}}}\right)_{\alpha}^{\beta}\left(\nabla_{\beta} L\right)+i \frac{1}{5}\left(\sigma_{\underline{b}}\right)^{\beta \delta}\left(\nabla_{\delta} L\right) T_{\alpha \beta^{\underline{c}}} \\
& +i \frac{1}{5}\left(\sigma_{\underline{b}}\right)^{\dot{\beta} \dot{\delta}}\left(\nabla_{\dot{\delta}} L\right) T_{\alpha \dot{\beta}} \underline{\underline{c}},
\end{aligned}
$$

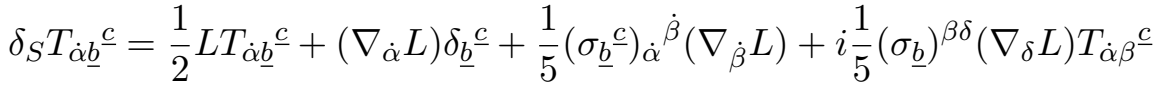

$$
\begin{aligned}
& +i \frac{1}{5}\left(\sigma_{\underline{b}}\right)^{\dot{\beta} \dot{\delta}}\left(\nabla_{\dot{\delta}} L\right) T_{\dot{\alpha} \dot{\beta}} \underline{\underline{c}} \\
& \delta_{S} T_{\alpha \underline{b}}^{\gamma}=L T_{\alpha \underline{b}}^{\gamma}+i \frac{1}{5} T_{\alpha \underline{\underline{b}}} \underline{c}\left(\sigma_{\underline{c}}\right)^{\gamma \delta}\left(\nabla_{\delta} L\right)-\frac{1}{2}\left(\nabla_{\underline{b}} L\right) \delta_{\alpha}^{\gamma}-i \frac{1}{5}\left(\sigma_{\underline{b}}\right)^{\gamma \beta}\left(\nabla_{\alpha} \nabla_{\beta} L\right) \\
& +i \frac{1}{5}\left(\sigma_{\underline{b}}\right)^{\delta \beta}\left(\nabla_{\delta} L\right) T_{\alpha \beta}^{\gamma}+i \frac{1}{5}\left(\sigma_{\underline{b}}\right)^{\dot{\delta} \dot{\beta}}\left(\nabla_{\dot{\delta}} L\right) T_{\alpha \dot{\beta}}{ }^{\gamma}+\frac{1}{2}\left(\sigma_{\underline{b}}^{\underline{c}}\right)_{\alpha}^{\gamma}\left(\nabla_{\underline{c}} L\right), \\
& \delta_{S} T_{\alpha \underline{b}} \dot{\gamma}=L T_{\alpha \underline{b}} \dot{\gamma}-i \frac{1}{5}\left(\sigma_{\underline{b}}\right)^{\dot{\gamma} \dot{\beta}}\left(\nabla_{\alpha} \nabla_{\dot{\beta}} L\right)+i \frac{1}{5}\left(\sigma_{\underline{b}}\right)^{\delta \beta}\left(\nabla_{\delta} L\right) T_{\alpha \beta} \dot{\gamma}+i \frac{1}{5}\left(\sigma_{\underline{b}}\right)^{\dot{\delta} \dot{\beta}}\left(\nabla_{\dot{\delta}} L\right) T_{\alpha \dot{\beta}} \dot{\gamma} \\
& -i \frac{1}{5}\left(\sigma_{\underline{c}}\right)^{\dot{\delta} \dot{\gamma}}\left(\nabla_{\dot{\delta}} L\right) T_{\alpha \underline{b}}{ }^{\underline{c}}, \\
& \delta_{S} T_{\dot{\alpha} \underline{\underline{b}}}^{\gamma}=L T_{\dot{\alpha} \underline{b}}^{\gamma}-i \frac{1}{5}\left(\sigma_{\underline{b}}\right)^{\gamma \beta}\left(\nabla_{\dot{\alpha}} \nabla_{\beta} L\right)+i \frac{1}{5}\left(\sigma_{\underline{b}}\right)^{\delta \beta}\left(\nabla_{\delta} L\right) T_{\dot{\alpha} \beta}^{\gamma}+i \frac{1}{5}\left(\sigma_{\underline{b}}\right)^{\dot{\delta} \dot{\beta}}\left(\nabla_{\dot{\delta}} L\right) T_{\dot{\alpha} \dot{\beta}}{ }^{\gamma} \\
& -i \frac{1}{5}\left(\sigma_{\underline{c}}\right)^{\delta \gamma}\left(\nabla_{\delta} L\right) T_{\dot{\alpha} \underline{b}}^{\underline{c}}, \\
& \delta_{S} T_{\dot{\alpha} \underline{b}} \underline{\dot{\gamma}}=L T_{\dot{\alpha} \underline{b}}^{\dot{\gamma}}+i \frac{1}{5} T_{\dot{\alpha} \underline{b}}^{\underline{c}}\left(\sigma_{\underline{c}}\right)^{\dot{\gamma} \dot{\delta}}\left(\nabla_{\dot{\delta}} L\right)-\frac{1}{2}\left(\nabla_{\underline{b}} L\right) \delta_{\dot{\alpha}}^{\dot{\gamma}}-i \frac{1}{5}\left(\sigma_{\underline{b}}\right)^{\dot{\gamma} \dot{\beta}}\left(\nabla_{\dot{\alpha}} \nabla_{\dot{\beta}} L\right) \\
& +i \frac{1}{5}\left(\sigma_{\underline{b}}\right)^{\delta \beta}\left(\nabla_{\delta} L\right) T_{\dot{\alpha} \beta} \dot{\gamma}+i \frac{1}{5}\left(\sigma_{\underline{b}}\right)^{\dot{\delta} \dot{\beta}}\left(\nabla_{\dot{\delta}} L\right) T_{\dot{\alpha} \dot{\beta}} \dot{\gamma}+\frac{1}{2}\left(\sigma_{\underline{b}}\right)_{\dot{c}} \dot{\gamma}^{\dot{\gamma}}\left(\nabla_{\underline{c}} L\right), \\
& \delta_{S} T_{\underline{a} b} \underline{\underline{c}}=L T_{\underline{a} b} \underline{\underline{c}}-i \frac{1}{5}\left(\sigma_{[\underline{a}}\right)^{\alpha \beta}\left(\nabla_{\alpha} L\right) T_{\beta \mid \underline{b}]^{\underline{c}}}-i \frac{1}{5}\left(\sigma_{[\underline{a}}\right)^{\dot{\alpha} \dot{\beta}}\left(\nabla_{\dot{\alpha}} L\right) T_{\dot{\beta} \mid \underline{b}]^{\underline{c}}}, \\
& \delta_{S} R_{\alpha \beta^{\underline{d}}}{ }^{\underline{d e}}=L R_{\alpha \beta^{\underline{\underline{d}}}}-T_{\alpha \beta^{[\underline{d}}}\left(\nabla^{\underline{e}]} L\right)+\frac{1}{5} T_{\alpha \beta^{\gamma}}\left(\sigma^{\underline{\underline{d}} e}\right)_{\gamma}{ }^{\delta}\left(\nabla_{\delta} L\right)+\frac{1}{5} T_{\alpha \beta^{\dot{\gamma}}}\left(\sigma^{\underline{d} e}\right)_{\dot{\gamma}}^{\dot{\delta}}\left(\nabla_{\dot{\delta}} L\right) \\
& -\frac{1}{5}\left(\sigma^{\underline{d} e}\right)_{(\alpha}{ }^{\delta}\left(\nabla_{\beta} \nabla_{\delta} L\right),
\end{aligned}
$$

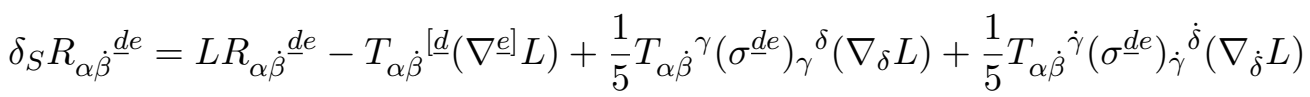

$$
\begin{aligned}
& -\frac{1}{5}\left(\sigma^{\underline{d} e}\right)_{\alpha}{ }^{\delta}\left(\nabla_{\dot{\beta}} \nabla_{\delta} L\right)-\frac{1}{5}\left(\sigma^{\underline{d} e}\right)_{\dot{\beta}}{ }^{\dot{\gamma}}\left(\nabla_{\alpha} \nabla_{\dot{\gamma}} L\right),
\end{aligned}
$$

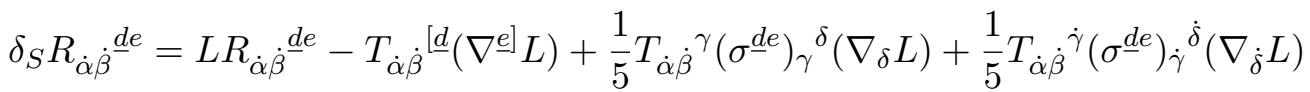

$$
\begin{aligned}
& -\frac{1}{5}\left(\sigma^{\underline{d} e}\right)_{(\dot{\alpha}}^{\dot{\delta}}\left(\nabla_{\dot{\beta})} \nabla_{\dot{\delta}} L\right) \text {. }
\end{aligned}
$$


Similar to 11D, we define $\mathcal{J}$-tensors and use them to construct the Weyl tensor superfield. The $\mathcal{J}$-tensors constructed are the following,

$$
\begin{array}{ll}
\mathcal{J}_{\alpha}^{(1)}=\frac{1}{2} T_{\alpha \beta}{ }^{\beta}, & \mathcal{J}_{\dot{\alpha}}^{(1)}=\frac{1}{2} T_{\dot{\alpha} \dot{\beta}}{ }^{\dot{\beta}}, \\
\mathcal{J}_{\alpha}^{(2)}=\frac{1}{8} T_{\alpha \dot{\beta}} \dot{\beta}, & \mathcal{J}_{\dot{\alpha}}^{(2)}=\frac{1}{8} T_{\dot{\alpha} \beta}{ }^{\beta}, \\
\mathcal{J}_{\alpha}^{(3)}=\frac{1}{8} T_{\alpha \underline{\underline{b}} \underline{ }}, & \mathcal{J}_{\dot{\alpha}}^{(3)}=\frac{1}{8} T_{\dot{\alpha} \underline{\underline{b}}} .
\end{array}
$$

Thus we see

$$
\begin{aligned}
& \delta_{S} \mathcal{J}_{\alpha}^{(1)}=\frac{1}{2} L \mathcal{J}_{\alpha}^{(1)}+\left(\nabla_{\alpha} L\right), \quad \delta_{S} \mathcal{J}_{\dot{\alpha}}^{(1)}=\frac{1}{2} L \mathcal{J}_{\dot{\alpha}}^{(1)}+\left(\nabla_{\dot{\alpha}} L\right), \\
& \delta_{S} \mathcal{J}_{\alpha}^{(2)}=\frac{1}{2} L \mathcal{J}_{\alpha}^{(2)}+\left(\nabla_{\alpha} L\right), \quad \delta_{S} \mathcal{J}_{\dot{\alpha}}^{(2)}=\frac{1}{2} L \mathcal{J}_{\dot{\alpha}}^{(2)}+\left(\nabla_{\dot{\alpha}} L\right), \\
& \delta_{S} \mathcal{J}_{\alpha}^{(3)}=\frac{1}{2} L \mathcal{J}_{\alpha}^{(3)}+\left(\nabla_{\alpha} L\right), \quad \delta_{S} \mathcal{J}_{\dot{\alpha}}^{(3)}=\frac{1}{2} L \mathcal{J}_{\dot{\alpha}}^{(3)}+\left(\nabla_{\dot{\alpha}} L\right) .
\end{aligned}
$$

One can see there are only two independent objects (one from each copy of Grassmann coordinates) by changing basis for these superfields. Define

$$
\begin{aligned}
\mathcal{J}_{\alpha}^{(+)} & =\frac{1}{3}\left(\mathcal{J}_{\alpha}^{(3)}+\mathcal{J}_{\alpha}^{(1)}+\mathcal{J}_{\alpha}^{(2)}\right), & \mathcal{J}_{\dot{\alpha}}^{(+)} & =\frac{1}{3}\left(\mathcal{J}_{\dot{\alpha}}^{(3)}+\mathcal{J}_{\dot{\alpha}}^{(1)}+\mathcal{J}_{\dot{\alpha}}^{(2)}\right), \\
\mathcal{J}_{\alpha}^{(-1)} & =\frac{1}{2}\left(\mathcal{J}_{\alpha}^{(3)}-\mathcal{J}_{\alpha}^{(1)}\right), & \mathcal{J}_{\dot{\alpha}}^{(-1)} & =\frac{1}{2}\left(\mathcal{J}_{\dot{\alpha}}^{(3)}-\mathcal{J}_{\dot{\alpha}}^{(1)}\right), \\
\mathcal{J}_{\alpha}^{(-2)} & =\frac{1}{6}\left(\mathcal{J}_{\alpha}^{(3)}+\mathcal{J}_{\alpha}^{(1)}-2 \mathcal{J}_{\alpha}^{(2)}\right), & \mathcal{J}_{\dot{\alpha}}^{(-2)} & =\frac{1}{6}\left(\mathcal{J}_{\dot{\alpha}}^{(3)}+\mathcal{J}_{\dot{\alpha}}^{(1)}-2 \mathcal{J}_{\dot{\alpha}}^{(2)}\right) .
\end{aligned}
$$

The variations of these $\mathcal{J}$-tensors under the scale variation take the forms

$$
\begin{aligned}
\delta_{S} \mathcal{J}_{\alpha}^{(+)} & =\frac{1}{2} L \mathcal{J}_{\alpha}^{(+)}+\left(\nabla_{\alpha} L\right), & \delta_{S} \mathcal{J}_{\dot{\alpha}}^{(+)} & =\frac{1}{2} L \mathcal{J}_{\dot{\alpha}}^{(+)}+\left(\nabla_{\dot{\alpha}} L\right), \\
\delta_{S} \mathcal{J}_{\alpha}^{(-1)} & =\frac{1}{2} L \mathcal{J}_{\alpha}^{(-1)}, & \delta_{S} \mathcal{J}_{\dot{\alpha}}^{(-1)} & =\frac{1}{2} L \mathcal{J}_{\dot{\alpha}}^{(-1)}, \\
\delta_{S} \mathcal{J}_{\alpha}^{(-2)} & =\frac{1}{2} L \mathcal{J}_{\alpha}^{(-2)}, & \delta_{S} \mathcal{J}_{\dot{\alpha}}^{(-2)} & =\frac{1}{2} L \mathcal{J}_{\dot{\alpha}}^{(-2)}
\end{aligned}
$$

In this basis, only $\mathcal{J}_{\alpha}^{(+)}$and $\mathcal{J}_{\dot{\alpha}}^{(+)}$serve as spinorial gauge connections. The Weyl tensor is then constructed using conformal weight 1 torsion supertensors and conformal weight $\frac{1}{2}$ spinorial gauge connections. By requiring $\delta_{S} \mathcal{W}_{\underline{a} b c}=L \mathcal{W}_{\underline{a} b c}$, we obtain

$$
\begin{aligned}
\mathcal{W}_{\underline{a b c}}= & \frac{1}{32}\left\{\left(\sigma^{\underline{d}}\right)_{\gamma \beta}\left(\sigma_{\underline{a} b c}\right)^{\beta \alpha} T_{\alpha \underline{d}}^{\gamma}-i 2\left(\sigma_{\underline{a} b c}\right)^{\alpha \beta}\left[\nabla_{\alpha} \mathcal{J}_{\beta}^{(+)}-\frac{6}{5} \mathcal{J}_{\alpha}^{(+)} \mathcal{J}_{\beta}^{(+)}\right]\right. \\
& \left.+\left(\sigma^{\underline{d}}\right)_{\dot{\gamma} \dot{\beta}}\left(\sigma_{\underline{a} b c}\right)^{\dot{\beta} \dot{\alpha}} T_{\dot{\alpha} \underline{d}}^{\dot{\gamma}}-i 2\left(\sigma_{\underline{a} b c}\right)^{\dot{\alpha} \dot{\beta}}\left[\nabla_{\dot{\alpha}} \mathcal{J}_{\dot{\beta}}^{(+)}-\frac{6}{5} \mathcal{J}_{\dot{\alpha}}^{(+)} \mathcal{J}_{\dot{\beta}}^{(+)}\right]\right\} .
\end{aligned}
$$

\section{$3.210 \mathrm{D}, \mathcal{N}=$ IIB supergravity derivatives \& scale transformations}

Now, the covariant derivative operators linear in the complex conformal compensator $\Psi$ (appropriate for the IIB theory) and necessary for a Nordström theory (Equations (8.92)- 
(8.94) in [22]) may be given by

$$
\begin{aligned}
\nabla_{\alpha}= & \mathrm{D}_{\alpha}+\frac{1}{2} \Psi \mathrm{D}_{\alpha}+\frac{1}{10}\left(\sigma^{\underline{a} b}\right)_{\alpha}^{\beta}\left(\mathrm{D}_{\beta} \Psi\right) \mathcal{M}_{\underline{a} b}, \\
\bar{\nabla}_{\alpha}= & \overline{\mathrm{D}}_{\alpha}+\frac{1}{2} \bar{\Psi}_{\alpha}+\frac{1}{10}\left(\sigma^{\underline{a} b}\right)_{\alpha}^{\beta}\left(\overline{\mathrm{D}}_{\beta} \bar{\Psi}\right) \mathcal{M}_{\underline{a} b}, \\
\nabla_{\underline{a}}= & \partial_{\underline{a}}+\frac{1}{2}(\Psi+\bar{\Psi}) \partial_{\underline{a}}-i \frac{1}{32}\left(\sigma_{\underline{a}}\right)^{\alpha \beta}\left[\overline{\mathrm{D}}_{\alpha}\left(\Psi+\frac{27}{5} \bar{\Psi}\right)\right] \mathrm{D}_{\beta} \\
& -i \frac{1}{32}\left(\sigma_{\underline{a}}\right)^{\alpha \beta}\left[\mathrm{D}_{\alpha}\left(\bar{\Psi}+\frac{27}{5} \Psi\right)\right] \overline{\mathrm{D}}_{\beta}-\frac{1}{2}\left[\partial_{\underline{c}}(\Psi+\bar{\Psi})\right] \mathcal{M}_{\underline{\underline{a}}},
\end{aligned}
$$

here the "bare" algebra of the supersymmetry covariant derivatives and the spacetime partial derivative takes the forms

$$
\left\{\mathrm{D}_{\alpha}, \mathrm{D}_{\beta}\right\}=0, \quad\left\{\overline{\mathrm{D}}_{\alpha}, \overline{\mathrm{D}}_{\beta}\right\}=0, \quad\left\{\mathrm{D}_{\alpha}, \overline{\mathrm{D}}_{\beta}\right\}=i\left(\sigma^{\underline{a}}\right)_{\alpha \beta} \partial_{\underline{a}},
$$

which indicate the non-vanishing torsion supertensors must be given by

$$
T_{\alpha \beta^{\underline{c}}}=0, \quad T_{\bar{\alpha} \bar{\beta}^{c}}=0, \quad T_{\alpha \bar{\beta}} \underline{c}=i\left(\sigma^{\underline{c}}\right)_{\alpha \beta} .
$$

By repeating the steps used to obtain (3.6), (3.7), and (3.8) in the last section, here we find,

$$
\begin{aligned}
\delta_{S} \nabla_{\alpha}= & \frac{1}{2} L \nabla_{\alpha}+\frac{1}{10}\left(\sigma^{\underline{a}}\right)_{\alpha}{ }^{\beta}\left(\nabla_{\beta} L\right) \mathcal{M}_{\underline{a} b}, \\
\delta_{S} \bar{\nabla}_{\alpha}= & \frac{1}{2} \bar{L}_{\alpha}+\frac{1}{10}\left(\sigma^{\underline{a} b}\right)_{\alpha}^{\beta}\left(\bar{\nabla}_{\beta} \bar{L}\right) \mathcal{M}_{\underline{a} b}, \\
\delta_{S} \nabla_{\underline{a}}= & \frac{1}{2}(L+\bar{L}) \nabla_{\underline{a}}-i \frac{1}{32}\left(\sigma_{\underline{a}}\right)^{\alpha \beta}\left[\bar{\nabla}_{\alpha}\left(L+\frac{27}{5} \bar{L}\right)\right] \nabla_{\beta} \\
& -i \frac{1}{32}\left(\sigma_{\underline{a}}\right)^{\alpha \beta}\left[\nabla_{\alpha}\left(\bar{L}+\frac{27}{5} L\right)\right] \bar{\nabla}_{\beta}-\frac{1}{2}\left[\nabla_{\underline{c}}(L+\bar{L})\right] \mathcal{M}_{\underline{a}^{\underline{c}}} .
\end{aligned}
$$

The super-Weyl parameter in (3.6), (3.7), and (3.8) is distinguished from that in (3.39), (3.40), and (3.41). The former involve a real scaling parameter superfield $L=\bar{L}$, whereas the latter involve a complex scaling parameter superfield $L \neq \bar{L}$. This means the transformation laws in (3.39), (3.40), and (3.41) imply both a real scaling parametrized by $\frac{1}{2}(L+\bar{L})$ and also a $\mathrm{U}(1)$ rotation parametrized by $i \frac{1}{2}(L-\bar{L})$. The terms proportional to $\mathcal{M}$ in (3.39), and (3.40) as well as the ones proportional to the spinorial derivatives in (3.41) depend on both the real superspace Weyl scaling parameter $\mathrm{u}=\frac{1}{2}(L+\bar{L})$ and the real $\mathrm{U}(1)$ rotation parameter $\mathrm{v}=-i \frac{1}{2}(L-\bar{L})$. To see this, one need only note $L=(\mathrm{u}+i \mathrm{v})$ and $\bar{L}=(\mathrm{u}-i \mathrm{v})$ in the latter equations.

Upon using the definitions of torsion and curvature tensors

$$
\begin{aligned}
& {\left[\nabla_{\alpha}, \nabla_{\beta}\right\}=T_{\alpha \beta}{ }^{\underline{c}} \nabla_{\underline{c}}+T_{\alpha \beta}{ }^{\gamma} \nabla_{\gamma}+T_{\alpha \beta}{ }^{\bar{\gamma}} \bar{\nabla}_{\gamma}+\frac{1}{2} R_{\alpha \beta} \underline{d}^{\underline{e}} \mathcal{M}_{\underline{\underline{e}}}^{\underline{d}},} \\
& {\left[\nabla_{\alpha}, \bar{\nabla}_{\beta}\right\}=T_{\alpha \bar{\beta}^{c}} \nabla_{\underline{c}}+T_{\alpha \bar{\beta}}{ }^{\gamma} \nabla_{\gamma}+T_{\alpha \bar{\beta}}{ }^{\bar{\gamma}} \bar{\nabla}_{\gamma}+\frac{1}{2} R_{\alpha \bar{\beta} \underline{\underline{d}}}{ }^{\underline{e}} \mathcal{M}_{\underline{\underline{e}}}^{\underline{d}},}
\end{aligned}
$$

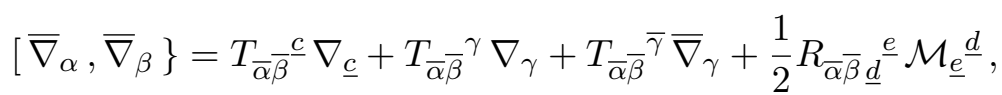




$$
\begin{aligned}
& {\left[\nabla_{\alpha}, \nabla_{\underline{b}}\right\}=T_{\alpha \underline{b}} \nabla_{\underline{c}}+T_{\alpha \underline{b}}{ }^{\gamma} \nabla_{\gamma}+T_{\alpha \underline{b}}{ }^{\bar{\gamma}} \bar{\nabla}_{\gamma}+\frac{1}{2} R_{\alpha \underline{b} \underline{d}^{\underline{e}}} \mathcal{M}_{\underline{\underline{e}} \underline{\underline{d}}},} \\
& {\left[\bar{\nabla}_{\alpha}, \nabla_{\underline{b}}\right\}=T_{\bar{\alpha} \underline{b}}^{\underline{c}} \nabla_{\underline{c}}+T_{\bar{\alpha} \underline{b}}^{\gamma} \nabla_{\gamma}+T_{\bar{\alpha} \underline{b}}{ }^{\bar{\gamma}} \bar{\nabla}_{\gamma}+\frac{1}{2} R_{\bar{\alpha} \underline{b} \underline{d}^{\underline{e}}} \mathcal{M}_{\underline{e}}^{\underline{d}},} \\
& {\left[\nabla_{\underline{a}}, \nabla_{\underline{b}}\right\}=T_{\underline{a} b}{ }^{\underline{c}} \nabla_{\underline{c}}+T_{\underline{a} b}{ }^{\gamma} \nabla_{\gamma}+T_{\underline{a b}}{ }^{\bar{\gamma}} \bar{\nabla}_{\gamma}+\frac{1}{2} R_{\underline{a} b} \underline{d}^{\underline{e}} \mathcal{M}_{\underline{e}}^{\underline{d}}, *}
\end{aligned}
$$

appropriate to the case of the $10 \mathrm{D}, \mathcal{N}=$ IIB theory, we find the Weyl scaling properties of all the superspace torsion and curvature supertensors with weights of less than three-halves as below. Weyl scaling properties of all other superspace torsion and curvature supertensors with weights of equal and larger than three-halves will be presented in appendix A.2.

$$
\begin{aligned}
& \delta_{S} T_{\alpha \beta} \underline{\underline{c}}=\frac{1}{2}(L-\bar{L}) T_{\alpha \beta^{\underline{c}}}, \\
& \delta_{S} T_{\alpha \bar{\beta}} \underline{c}=0 \text {, } \\
& \delta_{S} T_{\bar{\alpha} \bar{\beta}^{c}}=-\frac{1}{2}(L-\bar{L}) T_{\bar{\alpha} \bar{\beta}^{c}}, \\
& \delta_{S} T_{\alpha \beta} \bar{\gamma}=\left(L-\frac{1}{2} \bar{L}\right) T_{\alpha \beta} \bar{\gamma}+i T_{\alpha \beta} \underline{c}\left(\sigma_{\underline{c}}\right)^{\delta \gamma}\left(\nabla_{\delta}\left(\frac{1}{32} \bar{L}+\frac{27}{160} L\right)\right), \\
& \delta_{S} T_{\alpha \beta}{ }^{\gamma}=\frac{1}{2} L T_{\alpha \beta}^{\gamma}+i T_{\alpha \beta}{ }^{\underline{c}}\left(\sigma_{\underline{c}}\right)^{\gamma \delta}\left(\bar{\nabla}_{\delta}\left(\frac{1}{32} L+\frac{27}{160} \bar{L}\right)\right) \\
& \left.\left.+\frac{1}{2}\left(\nabla_{(\alpha} L\right) \delta_{\beta}\right)^{\gamma}+\frac{1}{20}\left(\sigma^{[2]}\right)_{(\alpha}{ }^{\delta}\left(\sigma_{[2]}\right)_{\beta}\right)^{\gamma}\left(\nabla_{\delta} L\right), \\
& \delta_{S} T_{\alpha \bar{\beta}} \bar{\gamma}^{\bar{\gamma}}=\frac{1}{2} L T_{\alpha \bar{\beta}} \bar{\gamma}^{\bar{\gamma}}+i T_{\alpha \bar{\beta}}{ }^{\underline{c}}\left(\sigma_{\underline{c}}\right)^{\delta \gamma}\left(\nabla_{\delta}\left(\frac{1}{32} \bar{L}+\frac{27}{160} L\right)\right)+\frac{1}{2}\left(\nabla_{\alpha} \bar{L}\right) \delta_{\beta}{ }^{\gamma} \\
& +\frac{1}{20}\left(\sigma^{[2]}\right)_{\beta}^{\gamma}\left(\sigma_{[2]}\right)_{\alpha}^{\delta}\left(\nabla_{\delta} L\right),
\end{aligned}
$$

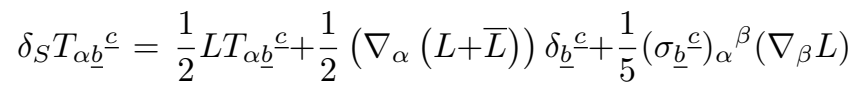

$$
\begin{aligned}
& +i\left(\sigma_{\underline{b}}\right)^{\delta \beta}\left(\nabla_{\delta}\left(\frac{1}{32} \bar{L}+\frac{27}{160} L\right)\right) T_{\alpha \bar{\beta}^{c}}+i\left(\sigma_{\underline{b}}\right)^{\delta \beta}\left(\bar{\nabla}_{\delta}\left(\frac{1}{32} L+\frac{27}{160} \bar{L}\right)\right) T_{\alpha \beta^{c}},
\end{aligned}
$$

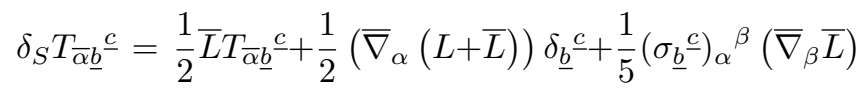

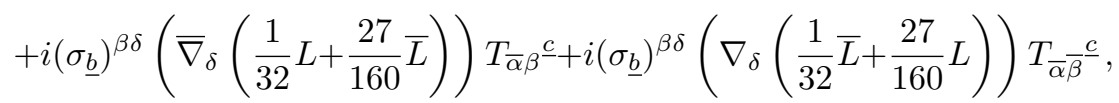

$$
\begin{aligned}
& \delta_{S} T_{\alpha \bar{\beta}}{ }^{\gamma}=\frac{1}{2} \bar{L} T_{\alpha \bar{\beta}}{ }^{\gamma}+i T_{\alpha \bar{\beta}}{ }^{c}\left(\sigma_{\underline{c}}\right)^{\delta \gamma}\left(\bar{\nabla}_{\delta}\left(\frac{1}{32} L+\frac{27}{160} \bar{L}\right)\right)+\frac{1}{2}\left(\bar{\nabla}_{\beta} L\right) \delta_{\alpha}{ }^{\gamma} \\
& +\frac{1}{20}\left(\sigma^{[2]}\right)_{\beta}^{\delta}\left(\sigma_{[2]}\right)_{\alpha}^{\gamma}\left(\bar{\nabla}_{\delta} \bar{L}\right) \text {, } \\
& \delta_{S} T_{\bar{\alpha} \bar{\beta}} \bar{\gamma}=\frac{1}{2} \bar{L} T_{\bar{\alpha} \bar{\beta}} \bar{\gamma}+i T_{\bar{\alpha} \bar{\beta}}{ }^{c}\left(\sigma_{\underline{c}}\right)^{\gamma \delta}\left(\nabla_{\delta}\left(\frac{1}{32} \bar{L}+\frac{27}{160} L\right)\right) \\
& \left.\left.+\frac{1}{2}\left(\bar{\nabla}_{(\alpha} \bar{L}\right) \delta_{\beta}\right)^{\gamma}+\frac{1}{20}\left(\sigma^{[2]}\right)_{(\alpha}^{\delta}\left(\sigma_{[2]}\right)_{\beta}\right)^{\gamma}\left(\bar{\nabla}_{\delta} \bar{L}\right) \text {, }
\end{aligned}
$$




$$
\begin{aligned}
& \delta_{S} T_{\bar{\alpha} \bar{\beta}^{\gamma}}=\left(\bar{L}-\frac{1}{2} L\right) T_{\bar{\alpha} \bar{\beta}}^{\gamma}+i T_{\bar{\alpha} \bar{\beta}} \underline{c}\left(\sigma_{\underline{c}}\right)^{\gamma \delta}\left(\bar{\nabla}_{\delta}\left(\frac{1}{32} L+\frac{27}{160} \bar{L}\right)\right) \\
& \delta_{S} T_{\alpha \underline{\underline{\gamma}}} \overline{\bar{\gamma}}=L T_{\alpha \underline{\underline{b}}}^{\bar{\gamma}}-i\left(\sigma_{\underline{b}}\right)^{\gamma \beta}\left(\nabla_{\alpha} \nabla_{\beta}\left(\frac{1}{32} \bar{L}+\frac{27}{160} L\right)\right)+i\left(\sigma_{\underline{b}}\right)^{\delta \beta}\left(\bar{\nabla}_{\delta}\left(\frac{1}{32} L+\frac{27}{160} \bar{L}\right)\right) T_{\alpha \beta} \bar{\gamma}^{\bar{\gamma}} \\
& +i\left(\sigma_{\underline{b}}\right)^{\delta \beta}\left(\nabla_{\delta}\left(\frac{1}{32} \bar{L}+\frac{27}{160} L\right)\right) T_{\alpha \bar{\beta}} \bar{\gamma}^{\bar{\gamma}}-i\left(\sigma_{\underline{c}}\right)^{\delta \gamma}\left(\nabla_{\delta}\left(\frac{1}{32} \bar{L}+\frac{27}{160} L\right)\right) T_{\alpha \underline{\underline{b}}}{ }^{\underline{c}}, \\
& \delta_{S} T_{\alpha \underline{\underline{b}}}{ }^{\gamma}=\frac{1}{2}(L+\bar{L}) T_{\alpha \underline{\underline{b}}}{ }^{\gamma}+i T_{\alpha \underline{\underline{b}}} \underline{\underline{c}}\left(\sigma_{\underline{c}}\right)^{\gamma \delta}\left(\bar{\nabla}_{\delta}\left(\frac{1}{32} L+\frac{27}{160} \bar{L}\right)\right)-\frac{1}{2}\left(\nabla_{\underline{b}} L\right) \delta_{\alpha}^{\gamma} \\
& -i\left(\sigma_{\underline{b}}\right)^{\gamma \beta}\left(\nabla_{\alpha} \bar{\nabla}_{\beta}\left(\frac{1}{32} L+\frac{27}{160} \bar{L}\right)\right)+i\left(\sigma_{\underline{b}}\right)^{\delta \beta}\left(\nabla_{\delta}\left(\frac{1}{32} \bar{L}+\frac{27}{160} L\right)\right) T_{\alpha \bar{\beta}^{\gamma}} \\
& +i\left(\sigma_{\underline{b}}\right)^{\delta \beta}\left(\bar{\nabla}_{\delta}\left(\frac{1}{32} L+\frac{27}{160} \bar{L}\right)\right) T_{\alpha \beta}{ }^{\gamma}+\frac{1}{4}\left(\sigma_{\underline{b}}\right)_{\alpha}^{\gamma}\left(\nabla_{\underline{c}}(L+\bar{L})\right),
\end{aligned}
$$

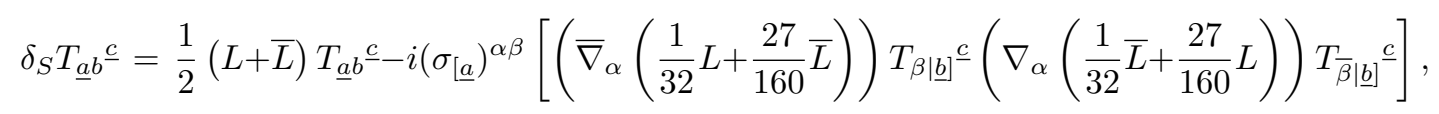

$$
\begin{aligned}
& \delta_{S} T_{\bar{\alpha} \underline{b}} \bar{\gamma}=\frac{1}{2}(L+\bar{L}) T_{\bar{\alpha} \underline{b}}{ }^{\bar{\gamma}}+i T_{\bar{\alpha} \underline{b}} \underline{c}\left(\sigma_{\underline{c}}\right)^{\gamma \delta}\left(\nabla_{\delta}\left(\frac{1}{32} \bar{L}+\frac{27}{160} L\right)\right)-\frac{1}{2}\left(\nabla_{\underline{b}} \bar{L}\right) \delta_{\alpha}^{\gamma} \\
& -i\left(\sigma_{\underline{b}}\right)^{\gamma \beta}\left(\bar{\nabla}_{\alpha} \nabla_{\beta}\left(\frac{1}{32} \bar{L}+\frac{27}{160} L\right)\right)+i\left(\sigma_{\underline{b}}\right)^{\delta \beta}\left(\bar{\nabla}_{\delta}\left(\frac{1}{32} L+\frac{27}{160} \bar{L}\right)\right) T_{\bar{\alpha} \beta} \bar{\gamma}
\end{aligned}
$$

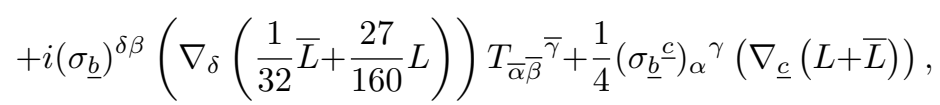

$$
\begin{aligned}
& \delta_{S} T_{\bar{\alpha} \underline{\underline{b}}}^{\gamma}=\bar{L} T_{\bar{\alpha} \underline{b}}^{\gamma}-i\left(\sigma_{\underline{b}}\right)^{\gamma \beta}\left(\bar{\nabla}_{\alpha} \bar{\nabla}_{\beta}\left(\frac{1}{32} L+\frac{27}{160} \bar{L}\right)\right)+i\left(\sigma_{\underline{b}}\right)^{\delta \beta}\left(\bar{\nabla}_{\delta}\left(\frac{1}{32} L+\frac{27}{160} \bar{L}\right)\right) T_{\bar{\alpha} \beta}{ }^{\gamma} \\
& +i\left(\sigma_{\underline{b}}\right)^{\delta \beta}\left(\nabla_{\delta}\left(\frac{1}{32} \bar{L}+\frac{27}{160} L\right)\right) T_{\bar{\alpha} \bar{\beta}}{ }^{\gamma}-i\left(\sigma_{\underline{c}}\right)^{\delta \gamma}\left(\bar{\nabla}_{\delta}\left(\frac{1}{32} L+\frac{27}{160} \bar{L}\right)\right) T_{\bar{\alpha} \underline{\underline{b}} \underline{\underline{c}},}
\end{aligned}
$$

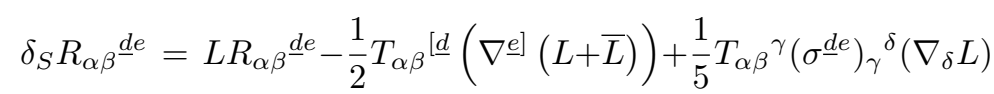

$$
\begin{aligned}
& \left.+\frac{1}{5} T_{\alpha \beta}{ }^{\bar{\gamma}}\left(\sigma^{\underline{d} e}\right)_{\gamma}^{\delta}\left(\bar{\nabla}_{\delta} \bar{L}\right)-\frac{1}{5}\left(\sigma^{-\underline{d e}}\right)_{\left(\alpha^{\delta}\right.}{ }^{\left(\nabla_{\beta}\right)} \nabla_{\delta} L\right), \\
& \left.\delta_{S} R_{\alpha \bar{\beta}} \underline{\underline{d} e}=\frac{1}{2}(L+\bar{L}) R_{\alpha \bar{\beta}} \underline{\underline{d}} e-\frac{1}{2} T_{\alpha \bar{\beta}} \underline{[d}\left(\nabla^{e}\right](L+\bar{L})\right)+\frac{1}{5} T_{\alpha \bar{\beta}^{\gamma}}\left(\sigma^{\underline{d} e}\right)_{\gamma}^{\delta}\left(\nabla_{\delta} L\right) \\
& +\frac{1}{5} T_{\alpha \bar{\beta}^{\bar{\gamma}}}\left(\sigma^{\underline{d} e}\right)_{\gamma}{ }^{\delta}\left(\bar{\nabla}_{\delta} \bar{L}\right)-\frac{1}{5}\left(\sigma^{\underline{d} e}\right)_{\alpha}{ }^{\delta}\left(\bar{\nabla}_{\beta} \nabla_{\delta} L\right)-\frac{1}{5}\left(\sigma^{\underline{d} e}\right)_{\beta}^{\gamma}\left(\nabla_{\alpha} \bar{\nabla}_{\gamma} \bar{L}\right), \\
& \delta_{S} R_{\bar{\alpha} \bar{\beta}^{\underline{d}}}=\bar{L} R_{\bar{\alpha} \bar{\beta}^{\underline{d}}}-\frac{1}{2} T_{\bar{\alpha} \bar{\beta}}{ }^{[\underline{d}}\left(\nabla^{e]}(L+\bar{L})\right)+\frac{1}{5} T_{\bar{\alpha} \bar{\beta}^{\gamma}}\left(\sigma^{\underline{d} e}\right)_{\gamma}^{\delta}\left(\nabla_{\delta} L\right) \\
& +\frac{1}{5} T_{\bar{\alpha} \bar{\beta}^{\bar{\gamma}}}\left(\sigma^{\underline{d} e}\right)_{\gamma}{ }^{\delta}\left(\bar{\nabla}_{\delta} \bar{L}\right)-\frac{1}{5}\left(\sigma^{-\underline{d e}}\right)_{(\alpha}{ }^{\delta}\left(\bar{\nabla}_{\beta)} \bar{\nabla}_{\delta} \bar{L}\right) .
\end{aligned}
$$

The grouping of terms in (3.43)-(3.61) is according to the real scale weight and $\mathrm{U}(1)$ charge. Their real scale weights and U(1) charges can be read off by expressing all the $L$ and $\bar{L}$ super parameters in terms of $\mathrm{u}$ and v. All this information is summarized in table 1 .

Again, we can construct $\mathcal{J}$-tensors in Type IIB theory. There are two independent ones (and their complex conjugates). This is because our scaling parameter superfield is 


\begin{tabular}{|c|c|c|c|}
\hline Torsion/Curvature & Scaling Coefficient & Real Scale Weight (u) & $\mathrm{U}(1)$ Charge (v) \\
\hline$T_{\alpha \beta^{\underline{c}}}$ & $\frac{1}{2}(L-\bar{L})$ & 0 & +1 \\
\hline$T_{\alpha \bar{\beta}^{\underline{c}}}$ & 0 & 0 & 0 \\
\hline$T_{\bar{\alpha} \bar{\beta}^{\underline{c}}}$ & $-\frac{1}{2}(L-\bar{L})$ & 0 & -1 \\
\hline$T_{\alpha \beta} \bar{\gamma}$ & $L-\frac{1}{2} \bar{L}$ & $\frac{1}{2}$ & $+\frac{3}{2}$ \\
\hline$T_{\alpha \beta} \gamma$ & $\frac{1}{2} L$ & $\frac{1}{2}$ & $+\frac{1}{2}$ \\
\hline$T_{\alpha \bar{\beta}} \bar{\gamma}$ & $\frac{1}{2} L$ & $\frac{1}{2}$ & $+\frac{1}{2}$ \\
\hline$T_{\alpha \underline{\underline{b}}} \underline{\underline{c}}$ & $\frac{1}{2} L$ & $\frac{1}{2}$ & $+\frac{1}{2}$ \\
\hline$T_{\bar{\alpha} \underline{b} \underline{c}^{\underline{c}}}$ & $\frac{1}{2} \bar{L}$ & $\frac{1}{2}$ & $-\frac{1}{2}$ \\
\hline$T_{\alpha \bar{\beta}}^{\gamma}$ & $\frac{1}{2} \bar{L}$ & $\frac{1}{2}$ & $-\frac{1}{2}$ \\
\hline$T_{\bar{\alpha} \bar{\beta}} \bar{\gamma}$ & $\frac{1}{2} \bar{L}$ & $\frac{1}{2}$ & $-\frac{1}{2}$ \\
\hline$T_{\bar{\alpha} \bar{\beta}^{\gamma}}^{\gamma}$ & $\bar{L}-\frac{1}{2} L$ & $\frac{1}{2}$ & $-\frac{3}{2}$ \\
\hline$T_{\alpha \underline{b}}{ }^{\bar{\gamma}}$ & $L$ & 1 & +1 \\
\hline$T_{\alpha \underline{b}}^{\gamma}$ & $\frac{1}{2}(L+\bar{L})$ & 1 & 0 \\
\hline$T_{\underline{a} b^{\underline{c}}}$ & $\frac{1}{2}(L+\bar{L})$ & 1 & 0 \\
\hline$T_{\bar{\alpha} \underline{b}}{ }^{\bar{\gamma}}$ & $\frac{1}{2}(L+\bar{L})$ & 1 & 0 \\
\hline$T_{\bar{\alpha} \underline{b}}^{\gamma}$ & $\bar{L}$ & 1 & -1 \\
\hline$R_{\alpha \beta^{\underline{d}}}$ & $L$ & 1 & +1 \\
\hline$R_{\alpha \bar{\beta}^{\underline{d}}} \underline{d e}$ & $\frac{1}{2}(L+\bar{L})$ & 1 & 0 \\
\hline$R_{\bar{a} \bar{\beta}} \underline{d} e$ & $\bar{L}$ & 1 & -1 \\
\hline
\end{tabular}

Table 1. Summary of Weyl complex scaling properties of $10 \mathrm{D}, \mathcal{N}=2 \mathrm{~B}$ torsions and curvatures.

complex and thus we have two degrees of freedom. The $\mathcal{J}$-tensors are defined by

$$
\begin{aligned}
& \mathcal{J}_{\alpha}^{(1)}=\frac{1}{4} T_{\alpha \beta}{ }^{\beta}, \quad \overline{\mathcal{J}}_{\alpha}^{(1)}=\frac{1}{4} T_{\bar{\alpha} \bar{\beta}}{ }^{\bar{\beta}}, \\
& \mathcal{J}_{\alpha}^{(2)}=\frac{5}{12} T_{\alpha \beta}{ }^{\beta}+\frac{1}{3} T_{\alpha \bar{\beta}} \overline{\bar{\beta}}^{\overline{3}}-\frac{1}{3} T_{\alpha \underline{\underline{b}} \underline{\underline{b}}}, \quad \overline{\mathcal{J}}_{\alpha}^{(2)}=\frac{5}{12} T_{\bar{\alpha} \bar{\beta}} \overline{\bar{\beta}}+\frac{1}{3} T_{\bar{\alpha} \beta}{ }^{\beta}-\frac{1}{3} T_{\bar{\alpha} \underline{\underline{b}}}^{\underline{\underline{b}}} .
\end{aligned}
$$

Their scale transformations are

$$
\begin{aligned}
& \delta_{S} \mathcal{J}_{\alpha}^{(1)}=\frac{1}{2} L \mathcal{J}_{\alpha}^{(1)}+\left(\nabla_{\alpha} L\right), \quad \delta_{S} \overline{\mathcal{J}}_{\alpha}^{(1)}=\frac{1}{2} \bar{L}_{\overline{\mathcal{J}}}^{(1)}+\left(\bar{\nabla}_{\alpha} \bar{L}\right), \\
& \delta_{S} \mathcal{J}_{\alpha}^{(2)}=\frac{1}{2} L \mathcal{J}_{\alpha}^{(2)}+\left(\nabla_{\alpha} \bar{L}\right), \quad \delta_{S} \overline{\mathcal{J}}_{\alpha}^{(2)}=\frac{1}{2} \bar{L} \overline{\mathcal{J}}_{\alpha}^{(2)}+\left(\bar{\nabla}_{\alpha} L\right) .
\end{aligned}
$$


The Weyl tensor can now be constructed from various $(\mathrm{u}, \mathrm{v})=(1,0)$ torsions, and combinations of $(\mathrm{u}, \mathrm{v})=\left(\frac{1}{2},+\frac{1}{2}\right)$ and $(\mathrm{u}, \mathrm{v})=\left(\frac{1}{2},-\frac{1}{2}\right)$ spinorial covariant derivatives and spinorial gauge connections such that each term has scaling properties $(\mathrm{u}, \mathrm{v})=(1,0)$. The final result is

$$
\begin{aligned}
\mathcal{W}_{\underline{a b c}}= & \frac{1}{32}\left\{\left(\sigma^{-}\right)_{\gamma \beta}\left(\sigma_{\underline{a} b c}\right)^{\beta \alpha} T_{\alpha \underline{\underline{d}}}^{\gamma}+\left(\sigma^{\underline{d}}\right)_{\gamma \beta}\left(\sigma_{\underline{a} b c}\right)^{\beta \alpha} T_{\bar{\alpha} \underline{\underline{d}}}{ }^{\bar{\gamma}}-4 T_{[\underline{a} b c]}\right. \\
& -i\left(\sigma_{\underline{a} b c}\right)^{\alpha \beta}\left[\frac{27}{16}\left(\nabla_{\alpha} \overline{\mathcal{J}}_{\beta}^{(1)}+\bar{\nabla}_{\alpha} \mathcal{J}_{\beta}^{(1)}\right)+\frac{5}{16}\left(\nabla_{\alpha} \overline{\mathcal{J}}_{\beta}^{(2)}+\bar{\nabla}_{\alpha} \mathcal{J}_{\beta}^{(2)}\right)\right. \\
& \left.\left.+\frac{1,863}{3,200} \mathcal{J}_{\alpha}^{(1)} \overline{\mathcal{J}}_{\beta}^{(1)}-\frac{33}{128} \mathcal{J}_{\alpha}^{(2)} \overline{\mathcal{J}}_{\beta}^{(2)}-\frac{411}{640}\left(\mathcal{J}_{\alpha}^{(1)} \overline{\mathcal{J}}_{\beta}^{(2)}+\mathcal{J}_{\alpha}^{(2)} \overline{\mathcal{J}}_{\beta}^{(1)}\right)\right]\right\} .
\end{aligned}
$$

\section{$3.310 \mathrm{D}, \mathcal{N}=1$ supergravity derivatives \& scale transformations}

The covariant derivatives linear in the conformal compensator $\Psi$ are given by (Equations (8.27)-(8.28) in [22])

$$
\begin{aligned}
& \nabla_{\alpha}=\mathrm{D}_{\alpha}+\frac{1}{2} \Psi \mathrm{D}_{\alpha}+\frac{1}{10}\left(\sigma^{\underline{a} b}\right)_{\alpha}^{\beta}\left(\mathrm{D}_{\beta} \Psi\right) \mathcal{M}_{\underline{a}}, \\
& \nabla_{\underline{a}}=\partial_{\underline{a}}+\Psi \partial_{\underline{a}}-i \frac{2}{5}\left(\sigma_{\underline{a}}\right)^{\alpha \beta}\left(\mathrm{D}_{\alpha} \Psi\right) \mathrm{D}_{\beta}-\left(\partial_{\underline{c}} \Psi\right) \mathcal{M}_{\underline{a}}^{\underline{\underline{c}}},
\end{aligned}
$$

where the $\mathcal{N}=1$ "D-operators" satisfy

$$
\left\{\mathrm{D}_{\alpha}, \mathrm{D}_{\beta}\right\}=i\left(\sigma^{\underline{a}}\right)_{\alpha \beta} \partial_{\underline{a}} .
$$

As the reader has seen the argument that follows from (3.65) and (3.66), thus we find

$$
\begin{aligned}
& \delta_{S} \nabla_{\alpha}=\frac{1}{2} L \nabla_{\alpha}+\frac{1}{10}\left(\sigma^{\underline{a} b}\right)_{\alpha}^{\beta}\left(\nabla_{\beta} L\right) \mathcal{M}_{\underline{a} b}, \\
& \delta_{S} \nabla_{\underline{a}}=L \nabla_{\underline{a}}-i \frac{2}{5}\left(\sigma_{\underline{a}}\right)^{\alpha \beta}\left(\nabla_{\alpha} L\right) \nabla_{\beta}-\left(\nabla_{\underline{c}} L\right) \mathcal{M}_{\underline{a}}^{\underline{\underline{c}}} .
\end{aligned}
$$

These results in (3.68) and (3.69) may be compared with the results derived in [7]. The two sets of Weyl scaling laws are different. However, this is due to the different choices of constraints that were chosen. Once this is taken into account, the scaling laws agree. The scaling laws used in [7] were presented as ansatzë, whereas the ones in (3.68) and (3.69) were derived from the Nordström supergravity results [22].

The definition of torsion and curvature tensors for the $10 \mathrm{D}, \mathcal{N}=1$ theory are identical in form to the equations given in (2.5). Thus, here we find repeating the series of calculations for $10 \mathrm{D}, \mathcal{N}=1$ superspace as was done for $11 \mathrm{D}, \mathcal{N}=1$ superspace yields the results that follow. Weyl scaling properties of all other superspace torsion and curvature supertensors with weights of equal and larger than three-halves will be presented in appendix A.3.

$$
\begin{aligned}
& \delta_{S} T_{\alpha \beta^{\underline{c}}}=0, \\
& \left.\delta_{S} T_{\alpha \beta}{ }^{\gamma}=\frac{1}{2} L T_{\alpha \beta}^{\gamma}+i \frac{2}{5} T_{\alpha \beta^{\underline{c}}}\left(\sigma_{\underline{c}}\right)^{\gamma \delta}\left(\nabla_{\delta} L\right)+\frac{1}{2}\left(\nabla_{(\alpha} L\right) \delta_{\beta}\right)^{\gamma} \\
& \left.+\frac{1}{20}\left(\sigma^{[2]}\right)_{(\alpha}^{\delta}\left(\sigma_{[2]}\right)_{\beta}\right)^{\gamma}\left(\nabla_{\delta} L\right) \text {, }
\end{aligned}
$$




$$
\begin{aligned}
& \delta_{S} T_{\alpha \underline{\underline{b}}} \underline{\underline{c}}=\frac{1}{2} L T_{\alpha \underline{\underline{b}}}^{\underline{c}}+\left(\nabla_{\alpha} L\right) \delta_{\underline{b}} \underline{\underline{c}}+\frac{1}{5}\left(\sigma_{\underline{b}}^{\underline{c}}\right)_{\alpha}^{\beta}\left(\nabla_{\beta} L\right) \\
& +i \frac{2}{5}\left(\sigma_{\underline{b}}\right)^{\beta \delta}\left(\nabla_{\delta} L\right) T_{\alpha \beta^{\underline{c}}}, \\
& \delta_{S} T_{\alpha \underline{b}}^{\gamma}=L T_{\alpha \underline{b}}^{\gamma}+i \frac{2}{5} T_{\alpha \underline{b}}{ }^{\underline{c}}\left(\sigma_{\underline{c}}\right)^{\gamma \delta}\left(\nabla_{\delta} L\right)-\frac{1}{2}\left(\nabla_{\underline{b}} L\right) \delta_{\alpha}^{\gamma} \\
& -i \frac{2}{5}\left(\sigma_{\underline{b}}\right)^{\gamma \beta}\left(\nabla_{\alpha} \nabla_{\beta} L\right)+i \frac{2}{5}\left(\sigma_{\underline{b}}\right)^{\delta \beta}\left(\nabla_{\delta} L\right) T_{\alpha \beta}{ }^{\gamma}+\frac{1}{2}\left(\sigma_{\underline{b}}{ }^{\underline{c}}\right)_{\alpha}^{\gamma}\left(\nabla_{\underline{c}} L\right), \\
& \delta_{S} T_{\underline{a}} b^{\underline{c}}=L T_{\underline{a} b^{\underline{c}}}-i \frac{2}{5}\left(\sigma_{[\underline{a}}\right)^{\alpha \beta}\left(\nabla_{\alpha} L\right) T_{\beta \mid \underline{b}]^{\underline{c}}}, \\
& \delta_{S} R_{\alpha \beta^{\underline{d}}} \underline{e}=L R_{\alpha \beta^{\underline{e}}}{ }^{\underline{d}}-T_{\alpha \beta}{ }^{[\underline{d}}\left(\nabla^{\underline{e}]} L\right)+\frac{1}{5} T_{\alpha \beta^{\gamma}}\left(\sigma^{\underline{d} e}\right)_{\gamma}^{\delta}\left(\nabla_{\delta} L\right) \\
& -\frac{1}{5}\left(\sigma^{\underline{d} e}\right)_{(\alpha}{ }^{\delta}\left(\nabla_{\beta} \nabla_{\delta} L\right) \text {. }
\end{aligned}
$$

Here we define the $\mathcal{J}$-tensors

$$
\mathcal{J}_{\alpha}^{(+)}=\frac{1}{6} T_{\alpha \underline{\underline{b}}}, \mathcal{J}_{\alpha}^{(-)}=T_{\alpha \beta}^{\beta},
$$

and they transform as

$$
\delta_{S} \mathcal{J}_{\alpha}^{(+)}=\frac{1}{2} L \mathcal{J}_{\alpha}^{(+)}+\left(\nabla_{\alpha} L\right), \delta_{S} \mathcal{J}_{\alpha}^{(-)}=\frac{1}{2} L \mathcal{J}_{\alpha}^{(-)} .
$$

Again, similar as in $11 \mathrm{D}, \mathcal{N}=1$ case, $\mathcal{J}_{\alpha}^{(-)}$transforms like a scale covariant tensor of weight $\frac{1}{2}$, the quantity $\mathcal{J}_{\alpha}^{(+)}$transforms with a scale weight of $\frac{1}{2}$ while being a spinorial gauge connection under a scaling transformation!

The necessary engineering dimension one superfield $\mathcal{W}_{\underline{a b c}}$ with Weyl weight one can be defined by

$$
\mathcal{W}_{\underline{a b c}}=\frac{1}{16}\left\{\left(\sigma^{\underline{d}}\right)_{\gamma \beta}\left(\sigma_{\underline{a b c}}\right)^{\beta \alpha} T_{\alpha \underline{d}}^{\gamma}-i 4\left(\sigma_{\underline{a} b c}\right)^{\alpha \beta}\left[\nabla_{\alpha} \mathcal{J}_{\beta}^{(+)}-\frac{22}{25} \mathcal{J}_{\alpha}^{(+)} \mathcal{J}_{\beta}^{(+)}\right]\right\}
$$

To our knowledge, the results presented in equations (3.33), (3.64), and (3.78) mark the first time that off-shell definitions of 10D Weyl superfield supergravity field strength tensors have been explicitly identified in the physics literature.

\section{A new methodology: Adynkras \& ADA scans}

Recently [23, 24], we have established a breakthrough approach which substantially lowered computational costs of determining how to embed a set of component fields within a superfield. Just as MRI (magnetic resonance imaging - based on the phenomenon of nuclear magnetic resonance) has brought amazing progress in creating high definition images of internal structures of bodies in biological domains, the approach in [23, 24] (which can be called "Adyndra Digital Analysis" or "ADA") permits the rapid assay of the Lorentz spectrum of component fields within superfields. 
There are a number of ways to define an "adynkra". ${ }^{7}$ One such meaning is as the name for a certain class of graphs. However, for our purposes here, we will use a definition tailored to $10 \mathrm{D}, \mathcal{N}=1 \mathrm{SUSY}$ where an adynkra is a collection of sets of Dynkin Labels that can be broken into subsets associated with a "Level number". The latter of these is an integer that takes on values from zero to sixteen. There exists a "generator" for such lists that takes the form

$\mathcal{G}=1 \oplus \ell\left\{(\square) \times\left[a_{1}, b_{1}, c_{1}, d_{1}, e_{1}\right]\right\} \oplus \bigoplus_{p=2}^{16} \frac{1}{p !}(\ell)^{p}\left\{\left(\square(\wedge \square)^{p-2} \wedge \square\right) \times\left[a_{p}, b_{p}, c_{p}, d_{p}, e_{p}\right]\right\}$,

which can be expanded to,

$$
\begin{aligned}
\mathcal{G}= & \ell\left\{(\square) \times\left[a_{1}, b_{1}, c_{1}, d_{1}, e_{1}\right]\right\} \\
& \oplus \bigoplus_{p=1}^{7} \frac{1}{p !}(\ell)^{2 p+1}\left\{\left(\square(\wedge \square)^{2 p-1} \wedge \square\right) \times\left[a_{2 p+1}, b_{2 p+1}, c_{2 p+1}, d_{2 p+1}, e_{2 p+1}\right]\right\} \\
& \oplus 1 \oplus \bigoplus_{p=1}^{8} \frac{1}{(2 p) !}(\ell)^{2 p}\left\{\left(\square(\wedge \square)^{2(p-1)} \wedge \square\right) \times\left[a_{2 p}, b_{2 p}, c_{2 p}, d_{2 p}, e_{2 p}\right]\right\},
\end{aligned}
$$

where $\left[a_{p}, b_{p}, c_{p}, d_{p}, e_{p}\right]$ (with $p=1, \ldots, 16$ ) denote Dynkin Labels appropriate for the fields over the 10-dimensional manifold. The "Level number" is the exponent seen associated with various powers of the level parameter $\ell$ in this expression. Finally $\square$ is a spinorial Young Tableau and $\wedge$ denotes the "wedge" product of the tableau. A more complete discussion of conventions and notation used in writing (4.1) can be found in [24]. All the terms on the first two lines of (4.2) are fermionic representations as the Young Tableau associated with $\square$ corresponds to the fundamental spinor representation of $\mathfrak{s o}(1,9)$. As the final terms have only even powers of $\square$ and due to the identity

$$
\square \wedge \square=\square=\square,
$$

these can be expressed solely in terms of YT's involving $\square$ (bosonic Young Tableaux), i.e. bosonic representations of $\mathfrak{s o}(1,9)$.

The generator (4.1) provides a basis for the creation of algorithms that are extraordinarily efficient at encoding representations of component fields contained within superfields. It is this efficiency that enabled unprecedented clarity about the component field contents of superfields in ten and eleven dimensions [23-25] and the computational tools we use are noted in [35-37].

The discussion in [24] is devoted to using the concepts of Branching Rules, Dynkin Labels, Plethysm, and Young Tableaux to calculate $\mathcal{G}$. In $[23,24]$ the basic "scalar" adynkra, denoted by $\mathcal{V}$, was calculated and described in detail. It is the foundational quantity expressed in terms of YT's. The computation expense to find all related quantities

\footnotetext{
${ }^{7}$ This is a word created from the concatenation of the works "adinkra" and "Dynkin" in an effort to denote the fusion of these two concepts.
} 
is substantially less than that needed to explicitly determine $\mathcal{V}$. This follows as higher representations are simply found from multiplication of $\mathcal{V}$ by Dynkin Labels when the latter are represented by YT's. Thus, we have

$$
\begin{aligned}
& \mathcal{V}_{[0,0,1,0,0]}=[0,0,1,0,0] \otimes \mathcal{V}, \mathcal{V}_{[1,0,1,0,1]}=[1,0,1,0,1] \otimes \mathcal{V}, \\
& \mathcal{V}_{[3,0,0,0,1]}=[3,0,0,0,1] \otimes \mathcal{V}, \mathcal{V}_{[4,0,0,0,0]}=[4,0,0,0,0] \otimes \mathcal{V},
\end{aligned}
$$

and these multiplications are straightforward by the use of the tools indicated in the references [35-37]. For any bosonic irrep $\mathcal{R}$ or fermionic irrep $\mathcal{R},(4.4) \mathrm{imples} \operatorname{dim}\left(\mathcal{V}_{\mathcal{R}}\right)=$ $\operatorname{dim}(\mathcal{R}) \operatorname{dim}(\mathcal{V})$ or $\operatorname{dim}\left(\mathcal{V}_{\mathcal{R}}\right)=\operatorname{dim}(\mathcal{R}) \operatorname{dim}(\mathcal{V})$, where $\operatorname{dim}(\mathcal{V})=65,536$. The Dynkin Label Library of the $\mathcal{V}$-superfield is explicitly shown below and similar results are shown in the appendix B for all superfields in (4.4).

- Level-0: $[0,0,0,0,0]$

- Level-1: $[0,0,0,1,0]$

- Level-2: $[0,0,1,0,0]$

- Level-3: $[0,1,0,0,1]$

- Level-4: $[0,2,0,0,0] \oplus[1,0,0,0,2]$

- Level-5: $[0,0,0,0,3] \oplus[1,1,0,0,1]$

- Level-6: $[0,1,0,0,2] \oplus[2,0,1,0,0]$

- Level-7: $[3,0,0,1,0] \oplus[1,0,1,0,1]$

- Level-8: $[4,0,0,0,0] \oplus[0,0,2,0,0] \oplus[2,0,0,1,1]$

- Level-9: $[3,0,0,0,1] \oplus[1,0,1,1,0]$

- Level-10: $[0,1,0,2,0] \oplus[2,0,1,0,0]$

- Level-11: $[0,0,0,3,0] \oplus[1,1,0,1,0]$

- Level-12: $[0,2,0,0,0] \oplus[1,0,0,2,0]$

- Level-13: $[0,1,0,1,0]$

- Level-14: $[0,0,1,0,0]$

- Level-15: $[0,0,0,0,1]$

- Level-16: $[0,0,0,0,0]$

The utility of such a library is straightforward.

For example, one could ask whether the component field corresponding to the bosonic irrep $[4,0,1,0,0]$ occurs within the $\theta$ expansion of $\mathcal{V}$ ? Upon examination of the library above, it is seen that the answer is, "no". In a similar manner, one can ask if the pair of 
representations given by $[0,2,0,0,0]$ and $[3,0,0,0,1]$ occur in the superfield $\mathcal{V}$ at adjacent levels with the fermionic one higher than the lower one? Once more a quick consultation of the library above returns the answer, "no". Clearly, more complicated questions of this nature can be pursued and we turn to this next.

\subsection{Component Dynkin Label examples \& ADA scans}

The component fermionic and bosonic representation content of the on-shell $10 \mathrm{D}, \mathcal{N}=1$ supergravity multiplet $[51,52]$ is given by

$$
\begin{aligned}
& \{\mathcal{F}\}_{\mathrm{SGI}}=\left(\chi_{\alpha},{\psi_{\underline{a}}}^{\alpha}\right)=([0,0,0,1,0],[1,0,0,0,1]), \\
& \{\mathcal{B}\}_{\mathrm{SGI}}=\left(\varphi, B_{\underline{a}} \underline{\underline{b}}, \mathrm{e}_{\underline{a}} \underline{\underline{m}}\right)=([0,0,0,0,0],[0,1,0,0,0],[2,0,0,0,0]),
\end{aligned}
$$

in its most common form. However, as first emphasized in the work of [56], there is an alternate version where the replacement $B_{\underline{a} \underline{b}} \rightarrow M_{\underline{a}_{1} \cdots \underline{a}_{6}}$ is compatible with the absence of anomalies [53, 54] as a realization of the LEEA of the heterotic string [55].

To complete $10 \mathrm{D}, \mathcal{N}=1$ supergravity into either the $10 \mathrm{D}, \mathcal{N}=$ IIA or IIB supergravity multiplets, it is necessary to find two other $10 \mathrm{D}, \mathcal{N}=1$ multiplets that contain the matter gravitino multiplets (MGM) discussed in appendix $\mathrm{C}$ of the work [59] and that indicated the existence of two such $10 \mathrm{D}, \mathcal{N}=1 \mathrm{MGM}$ systems. We may call one of them the "IIAMGM" system and the "IIBMGM" system. In terms of their component fermionic and bosonic representation contents, these look as

$$
\begin{aligned}
& \{\mathcal{F}\}_{\text {IIAMGM }}=\left(\chi_{\dot{\alpha}}, \psi_{\underline{a}}{ }^{\dot{\alpha}}\right)=([0,0,0,0,1],[1,0,0,1,0]), \\
& \{\mathcal{B}\}_{\text {IIAMGM }}=\left(B_{\underline{a}}, A_{\underline{a} \underline{b} \underline{c}}\right)=([1,0,0,0,0],[0,0,1,0,0]),
\end{aligned}
$$

and

$$
\begin{aligned}
& \{\mathcal{F}\}_{\text {IIBMGM }}=\left(\chi_{\alpha}^{\prime}, \psi_{\underline{a}}^{\prime \alpha}\right)=([0,0,0,1,0],[1,0,0,0,1]), \\
& \{\mathcal{B}\}_{\text {IIBMGM }}=\left(A, B_{\underline{a}}^{\prime} \underline{b}, A_{\underline{a}} \underline{b} \underline{c} \underline{d}\right)=([0,0,0,0,0],[0,1,0,0,0],[0,0,0,1,1]),
\end{aligned}
$$

respectively. It can be seen that the fermionic representations are presented in a manner where they are "higher" than the bosonic ones. This is due to the fact that dynamical fermionic fields possess higher engineering dimensions than dynamical bosonic fields. In terms of the "Level" numbers, the fermions are higher than the bosons.

Both the engineering dimensions and the Lorentz representations of all fields are key data inputs in the construction of Adynkra Digital Analysis (ADA) scans.

The adynkras for $\mathcal{V}, \mathcal{V}_{[0,0,1,0,0]}, \mathcal{V}_{[1,0,1,0,1]}, \mathcal{V}_{[3,0,0,0,1]}$, and $\mathcal{V}_{[4,0,0,0,0]}$ can be regarded as a set of "libraries". An ADA scan asks simple questions as any number of such queries can be asked. One is, "given the data of Level difference and the Dynkin Labels demonstrated in (4.6) does such a data pattern occur in the $\mathcal{V}$-library?" Another might be, "how many times does such a pattern occur?"

Going back to the work of [40], we have long asserted the interpretation of adinkras as being the analogs of genetic sequence content where superfields play the roles of biological bodies. An alternative would have been to analogize adinkras to quarks. However, this analogy suffers when one realizes the numbers of degrees of freedom of systems in ten and 
eleven dimensions (e.g. 11D SG possess 2,147,483,648 bosonic and the same number of fermionic degrees). So the number of possible targets in searchs are of more "biological" in order of magnitude than the numbers encountered in determing the quark content in hadronic spectroscopy problems.

Thus, the process of querying adynkra libraries more closely favors the challenges encountered in DNA analysis than the analysis of the quark spectra of hadronic matter. With modern IT platforms, both hardware and software, it is a straightforward matter to meet the challenges of writing codes to query such libraries... after they have been constructed. To focus this more accurately, the analogy is to regard the data (as given in (4.5), (4.6), and (4.7)) as primary biological sequence content, (e.g. similar in spirit to nucleotides of DNA/RNA or protein amino-acids). The adynkra "libraries" play the roles of genetic sequence data bases/libraries. ${ }^{8}$

\section{Toward the rest of the story}

The efforts we have described in sections 2 and 3 follow the "traditional" routes to understand supergravity in superspace where attention is focused on the "outside" variables, i.e. $\nabla_{\underline{A}}, T_{\underline{A}} \underline{B} \underline{C}$, and $R_{\underline{A}} \underline{B} \underline{c}^{\underline{d}}$. However, there is also a less trod pathway based on the study of the prepotentials, i.e. $\Psi, \mathcal{A}^{\underline{a}} \underline{\underline{b}}, \mathcal{N}_{\alpha}{ }^{\beta}$, and $\mathrm{H}_{\beta} \underline{\underline{b}}$. Really, due to the presence of constraints and covariance, only $\Psi$ and $\mathrm{H}_{\beta} \underline{\underline{b}}$ require deeper study. Heretofore, mostly the literature [1][21], has focused on the "outside" superfields rather than on the "inside" ones, $\Psi$, and $\mathrm{H}_{\beta} \underline{\underline{b}}$. The most obvious reason for this is the traditional approach requires a high computational price be paid to elucidate the $\theta$-expansion for component fields residing within the "inside" superfields.

As we wish to treat the cases of the $\mathcal{N}=1, \mathcal{N}=2 \mathrm{~A}$, and $\mathcal{N}=2 \mathrm{~B}$ uniformly, there is a conceptual approach available as an effective enabling strategy. We now turn to a discussion of this.

In the works of [41-45] there was initiated an approach where superfields with a higher realization of supersymmetry were formulated in terms of superfields that provide a lower realization of supersymmetry. In these cases, mostly superfields with $4 \mathrm{D}, \mathcal{N}=2$ SUSY were expressed in terms of superfields with $4 \mathrm{D}, \mathcal{N}=1$ SUSY. More recently [46-49], this approach has been implemented in the context of superfields with $11 \mathrm{D}, \mathcal{N}=1$ SUSY expressed in terms of superfields with $4 \mathrm{D}, \mathcal{N}=1 \mathrm{SUSY}$. Since we are working in the arena of $10 \mathrm{D}, \mathcal{N}=1, \mathcal{N}=2 \mathrm{~A}$, and $\mathcal{N}=2 \mathrm{~B}$ SUSY, the lower dimensional superfields in which to conduct our investigations are ones that realize $10 \mathrm{D}, \mathcal{N}=1$ SUSY.

To create a set of notational conventions that are graphically easy to follow in our subsequent discussion, we will denote the coordinates of $10 \mathrm{D}, \mathcal{N}=1$ superspace by $\left(x^{\underline{a}}, \theta^{\alpha}\right)$. In order to describe the superfields with $\mathcal{N}=2 \mathrm{~A}$, and $\mathcal{N}=2 \mathrm{~B}$ SUSY, we introduce a second Grassmann coordinate of the form $\theta^{\dot{\alpha}}$ in the case of the $\mathcal{N}=2 \mathrm{~A}$ superfields or of the form $\theta^{\alpha}$ in the case of the $\mathcal{N}=2 \mathrm{~B}$ superfields. Now we can continue to study the superfields $\mathcal{V}\left(x^{\underline{a}}, \theta^{\alpha}\right), \mathcal{V}_{\mathrm{IIA}}\left(x^{\underline{a}}, \theta^{\alpha}, \theta^{\dot{\alpha}}\right)$, or $\mathcal{V}_{\mathrm{IIB}}\left(x^{\underline{a}}, \theta^{\alpha}, \theta^{\alpha}\right)$ in each respective case. Next we treat these

\footnotetext{
${ }^{8}$ One example of such a bioinfomatics IT tool is the "basis local alignment search tool" (BLAST) [38] that serves this function.
} 
superfields, but in the latter two cases we use respective explicit expansions in terms of "green $\theta$ 's",

$$
\begin{aligned}
& \mathcal{V}_{\mathrm{IIA}}\left(x^{\underline{a}}, \theta^{\alpha}, \theta^{\dot{\alpha}}\right)=\mathcal{V}^{(0)}\left(x^{\underline{a}}, \theta^{\alpha}\right)+\theta^{\dot{\alpha}} \mathcal{V}_{\dot{\alpha}}^{(1)}\left(x^{\underline{a}}, \theta^{\alpha}\right)+\theta^{\dot{\alpha}} \theta^{\dot{\beta}} \mathcal{V}_{\dot{\alpha} \dot{\beta}}^{(2)}\left(x^{\underline{a}}, \theta^{\alpha}\right)+\ldots, \\
& \mathcal{V}_{\mathrm{IIB}}\left(x^{\underline{a}}, \theta^{\alpha}, \theta^{\alpha}\right)=\mathcal{V}^{(0)}\left(x^{\underline{a}}, \theta^{\alpha}\right)+\theta^{\alpha} \mathcal{V}_{\alpha}^{(1)}\left(x^{\underline{a}}, \theta^{\alpha}\right)+\theta^{\alpha} \theta^{\beta} \mathcal{V}_{\alpha \beta}^{(2)}\left(x^{\underline{a}}, \theta^{\alpha}\right)+\ldots,
\end{aligned}
$$

and where these expansions terminate at the sixteenth order of the "green $\theta$ 's". Clearly, these indicate respective sets of sixteen distinct $10 \mathrm{D}, \mathcal{N}=1$ superfields within the two types of $10 \mathrm{D}, \mathcal{N}=2$ superfields.

Although the two expansions in (5.1) and (5.2) appear rather similar, an interesting dichotomy emerges when ADA algorithms are applied to them. In the case of (5.1), it is seen for all the fermionic superfields, none contributes the conformal graviton representation to level-16 of Type IIA and IIB scalar superfields. In the case of (5.2), it is seen for all of the fermionic superfields except for $\mathcal{V}_{\alpha}, \mathcal{V}^{\alpha}, \mathcal{V}_{\left\{\underline{a}_{1} \underline{b}_{1} \underline{c}_{1} \underline{d}_{1} \underline{e}_{1}\right\}^{+}}{ }^{\alpha}$, and $\mathcal{V}_{\left\{\underline{a}_{1} \underline{b}_{1} \underline{c}_{1} \underline{d}_{1} \underline{e}_{1}\right\}^{-\alpha}}$, all contain the conformal graviton representation. This result suggests if we wish to find formulations of the Type-IIA and Type-IIB theories that possess a common truncation to the Type-I theory, we should eliminate the fermionic superfields in both expansions as subjects of additional study in this regard.

Now concentrating solely on the bosonic superfields in (5.1) and (5.2), we apply ADA algorithms to search within the bosonic $10 \mathrm{D}, \mathcal{N}=1$ superfields for the number $b_{\{1\}}$ of singlet irreps, the number $b_{\{45\}}$ of $\{45\}$ irreps, the number $b_{\{54\}}$ of $\{54\}$ irreps, the number $b_{\{210\}},{ }^{9}$ of $\{210\}$ irreps, the number $b_{\{\overline{16}\}}$ of $\{\overline{16}\}$ irreps, and the number $b_{\{\overline{144}\}}$ of $\{\overline{144}\}$ irreps. Furthermore, the ADA scans for these irreps within the bosonic $10 \mathrm{D}, \mathcal{N}=1$ superfields were designed additionally so that all bosonic irreps should occur at the same level in the superfield and also to fulfill the requirement that the $\{\overline{16}\}$ irrep and the $\{\overline{144}\}$ irrep should occur at one level higher than the bosonic irreps. The $10 \mathrm{D}, \mathcal{N}=2 \mathrm{~A}$ results are shown in the table 2 below.

Looking at this table, the reader's attention is directed to the Dynkin Labels. The distinct ones are $[0,0,1,0,0],[0,2,0,0,0],[1,0,0,2,0],[0,1,0,2,0],[2,0,1,0,0],[4,0,0,0,0]$, $[0,0,2,0,0],[2,0,0,1,1],[0,1,0,0,2]$, and $[1,0,0,0,2]$.

In a similar manner ADA scans can be applied to the bosonic $10 \mathrm{D}, \mathcal{N}=1$ superfields in the expansion shown in (5.2). In this case the sets of members of bosonic $10 \mathrm{D}, \mathcal{N}=1$ superfields that are found is identical to the ones found by starting from the expansion in (5.1). The $10 \mathrm{D}, \mathcal{N}=2 \mathrm{~B}$ results are shown in table 3 .

For all Type-I superfields shown in the two tables above, if there is $\{54\}$ appears in level- $n$, there exits $\{120\}$ irrep in level- $(n+2)$, which is the three (seven)-form (i.e. the field strength for either the $\{45\}$ or $\{210\}$ irreps). Also upon comparing the two tables, it is clear that the same two sets of $10 \mathrm{D}, \mathcal{N}=1$ superfields appear in both. The only differences that occur take place when a prepotential involves a 5 -form index. In these cases, the 5 -form indices are "flipped" between the $10 \mathrm{D}, \mathcal{N}=$ IIA and $10 \mathrm{D}, \mathcal{N}=$ IIB theories.

\footnotetext{
${ }^{9}$ The reason this irrep is considered in the search is due to the fact that it is known there is a duality "flip" [50] that exchanges the $b_{\{54\}}$ and $b_{\{210\}}$ irreps in $10 \mathrm{D}, \mathcal{N}=1$ supergravity and which is consistent with the anomaly-freedom conditions and Green-Schwarz $\sigma$-models associated with the low-energy heterotic string $[56,57]$ effective action.
} 


\begin{tabular}{|c|c|c|c|c|c|c|c|c|}
\hline Type-I Superfield & Dynkin Label & $b_{\{1\}}$ & $b_{\{45\}}$ & $b_{\{54\}}$ & $b_{\{210\}}$ & $b_{\{1 \overline{16}\}}$ & $b_{\{\overline{144}\}}$ & $\begin{array}{l}\text { Level of }\{54\} \text { in } \\
\text { Type-I Superfield }\end{array}$ \\
\hline $\mathcal{V}_{\left\{\underline{a}_{1} \underline{b}_{1} \underline{c}_{1}\right\}}$ & {$[0,0,1,0,0]$} & 1 & 1 & 1 & 2 & 1 & 1 & 14 \\
\hline $\mathcal{V}_{\left\{\underline{a}_{1} \underline{b}_{1}, \underline{a}_{2} \underline{b}_{2}\right\}}$ & {$[0,2,0,0,0]$} & 1 & 1 & 1 & 2 & 1 & 1 & 12 \\
\hline $\mathcal{V}_{\left\{\underline{a}_{2} \mid \underline{a}_{1} \underline{b}_{1} \underline{c}_{1} \underline{d}_{1} \underline{e}_{1}\right\}^{-}}$ & {$[1,0,0,2,0]$} & 0 & 0 & 1 & 1 & 0 & 1 & 12 \\
\hline $\mathcal{V}_{\left\{\underline{a}_{2} \underline{b}_{2} \mid \underline{a}_{1} \underline{b}_{1} \underline{c}_{1} \underline{d}_{1} \underline{e}_{1}\right\}^{-}}$ & {$[0,1,0,2,0]$} & 0 & 0 & 1 & 1 & 0 & 1 & 10 \\
\hline $\mathcal{V}_{\left\{\underline{a}_{2}, \underline{a}_{3} \mid \underline{a}_{1} \underline{b}_{1} \underline{c}_{1}\right\}}$ & {$[2,0,1,0,0]$} & 1 & 2 & 2 & 4 & 1 & 3 & 10 \\
\hline $\mathcal{V}_{\left\{\underline{a}_{1}, \underline{a}_{2}, \underline{a}_{3}, \underline{a}_{4}\right\}}$ & {$[4,0,0,0,0]$} & 1 & 1 & 1 & 1 & 1 & 1 & 8 \\
\hline $\mathcal{V}_{\left\{\underline{a}_{1} \underline{b}_{1} \underline{c}_{1}, \underline{a}_{2} \underline{b}_{2} \underline{c}_{2}\right\}}$ & {$[0,0,2,0,0]$} & 1 & 1 & 1 & 3 & 1 & 2 & 8 \\
\hline $\mathcal{V}_{\left\{\underline{a}_{2}, \underline{a}_{3} \mid \underline{a}_{1} \underline{b}_{1} \underline{c}_{1} \underline{d}_{1}\right\}}$ & {$[2,0,0,1,1]$} & 1 & 3 & 2 & 6 & 2 & 5 & 8 \\
\hline $\mathcal{V}_{\left\{\underline{a}_{2} \underline{b}_{2} \mid \underline{a}_{1} \underline{b}_{1} \underline{c}_{1} \underline{d}_{1} \underline{e}_{1}\right\}^{+}}$ & {$[0,1,0,0,2]$} & 0 & 0 & 1 & 1 & 0 & 1 & 6 \\
\hline $\mathcal{V}_{\left\{\underline{a}_{2}, \underline{a}_{3} \mid \underline{a}_{1} \underline{b}_{1} \underline{c}_{1}\right\}}$ & {$[2,0,1,0,0]$} & 1 & 2 & 2 & 4 & 2 & 4 & 6 \\
\hline $\mathcal{V}_{\left\{\underline{a}_{1} \underline{b}_{1}, \underline{a}_{2} \underline{b}_{2}\right\}}$ & {$[0,2,0,0,0]$} & 1 & 1 & 1 & 2 & 1 & 2 & 4 \\
\hline $\mathcal{V}_{\left\{\underline{a}_{2} \mid \underline{a}_{1} \underline{b}_{1} \underline{c}_{1} \underline{d}_{1} \underline{e}_{1}\right\}^{+}}$ & {$[1,0,0,0,2]$} & 0 & 0 & 1 & 1 & 0 & 1 & 4 \\
\hline $\mathcal{V}_{\left\{\underline{a}_{1} \underline{b}_{1} \underline{c}_{1}\right\}}$ & {$[0,0,1,0,0]$} & 1 & 1 & 1 & 2 & 1 & 2 & 2 \\
\hline
\end{tabular}

Table 2. Contributions of Each Type-I Superfield to the component bosonic and fermionic representation content of the $10 \mathrm{D}, \mathcal{N}=1$ supergravity multiplet at Level-16/17 of Type-IIA Scalar Superfield Decomposition.

The last column of the preceding two tables gives the height of the conformal graviton irrep in the Type-I superfield. This simultaneously gives the location of the Type-I superfield in the $\theta$-expansion. So for example the conformal graviton irrep indicated in the first row of either of the preceding tables is found at Level number 14. This means that the type-I superfield containing those conformal graviton irreps are located at quadratic order in the $\theta$-expansion.

We must exercise a note of caution in regards to our proposal, however. There is a long standing alternate proposal [58] for the $10 \mathrm{D}, \mathcal{N}=1$ superspace supergravity prepotential. This work has suggested that the $\{120\}$ irrep (the $[0,0,1,0,0]$ irrep or the superfield described by $\mathcal{V}_{\left\{\underline{a}_{1} \underline{b}_{1} \underline{c}_{1}\right\}}$, should play this role. One argument that mitigates against this concern is the fact that the $[0,0,1,0,0]$ irrep in the $10 \mathrm{D}$ theory does not connect to the unique conformal graviton seen in the 11D scalar supermultiplet.

In this section, we have looked at the issue of what superfield could serve as the $10 \mathrm{D}, \mathcal{N}=1$ supergravity prepotential. Our investigation began from the scalar superfield for both the Type-IIA and Type-IIB superspaces. The choice of the scalar superfield is motivated by our study of the $11 \mathrm{D}, \mathcal{N}=1$ superspace gravity theory [24] where the scalar superfield is the simplest superfield that contains the conformal graviton representation. In this context, it was found that the conformal graviton occurs exactly at the middle level of the superfield. A truncation of $11 \mathrm{D}, \mathcal{N}=1$ superspace to $10 \mathrm{D}, \mathcal{N}=$ IIA superspace must also then yield a scalar superfield as the supergravity prepotential. 


\begin{tabular}{|c|c|c|c|c|c|c|c|c|}
\hline Type-I Superfield & Dynkin Label & $b_{\{1\}}$ & $b_{\{45\}}$ & $b_{\{54\}}$ & $b_{\{210\}}$ & $b_{\{\overline{16}\}}$ & $b_{\{\overline{144}\}}$ & $\begin{array}{c}\text { Level of }\{54\} \text { in } \\
\text { Type-I Superfield }\end{array}$ \\
\hline $\mathcal{V}_{\left\{\underline{a}_{1} \underline{b}_{1} \underline{c}_{1}\right\}}$ & {$[0,0,1,0,0]$} & 1 & 1 & 1 & 2 & 1 & 1 & 14 \\
\hline $\mathcal{V}_{\left\{\underline{a}_{1} \underline{b}_{1}, \underline{a}_{2} \underline{b}_{2}\right\}}$ & {$[0,2,0,0,0]$} & 1 & 1 & 1 & 2 & 1 & 1 & 12 \\
\hline $\mathcal{V}_{\left\{\underline{a}_{2} \underline{a}_{1} \underline{b}_{1} \underline{c}_{1} \underline{d}_{1} \underline{e}_{1}\right\}^{+}}$ & {$[1,0,0,0,2]$} & 1 & 2 & 1 & 3 & 1 & 2 & 12 \\
\hline $\mathcal{V}_{\left\{\underline{a}_{2} \underline{b}_{2} \mid \underline{a}_{1} \underline{b}_{1} \underline{c}_{1} \underline{d}_{1} \underline{e}_{1}\right\}^{+}}$ & {$[0,1,0,0,2]$} & 1 & 2 & 1 & 4 & 2 & 3 & 10 \\
\hline $\mathcal{V}_{\left\{\underline{a}_{2}, \underline{a}_{3} \mid \underline{a}_{1} \underline{b}_{1} \underline{c}_{1}\right\}}$ & {$[2,0,1,0,0]$} & 1 & 2 & 2 & 4 & 1 & 3 & 10 \\
\hline $\mathcal{V}_{\left\{\underline{a}_{1}, \underline{a}_{2}, \underline{a}_{3}, \underline{a}_{4}\right\}}$ & {$[4,0,0,0,0]$} & 1 & 1 & 1 & 1 & 1 & 1 & 8 \\
\hline $\mathcal{V}_{\left\{\underline{a}_{1} \underline{b}_{1} \underline{c}_{1}, \underline{a}_{2} \underline{b}_{2} \underline{c}_{2}\right\}}$ & {$[0,0,2,0,0]$} & 1 & 1 & 1 & 3 & 1 & 2 & 8 \\
\hline $\mathcal{V}_{\left\{\underline{a}_{2}, \underline{a}_{3} \mid \underline{a}_{1} \underline{b}_{1} \underline{c}_{1} \underline{d}_{1}\right\}}$ & {$[2,0,0,1,1]$} & 1 & 3 & 2 & 6 & 2 & 5 & 8 \\
\hline $\mathcal{V}_{\left\{\underline{a}_{2} \underline{b}_{2} \mid \underline{a}_{1} \underline{b}_{1} \underline{c}_{1} \underline{d}_{1} \underline{e}_{1}\right\}^{-}}[0,1,0,2,0]$ & 1 & 2 & 1 & 4 & 1 & 3 & 6 \\
\hline $\mathcal{V}_{\left\{\underline{a}_{2}, \underline{a}_{3} \mid \underline{a}_{1} \underline{b}_{1} \underline{c}_{1}\right\}}$ & {$[2,0,1,0,0]$} & 1 & 2 & 2 & 4 & 2 & 4 & 6 \\
\hline $\mathcal{V}_{\left\{\underline{a}_{1} \underline{b}_{1}, \underline{a}_{2} \underline{b}_{2}\right\}}$ & {$[0,2,0,0,0]$} & 1 & 1 & 1 & 2 & 1 & 2 & 4 \\
\hline $\mathcal{V}_{\left\{\underline{a}_{2} \underline{a}_{1} \underline{b}_{1} \underline{c}_{1} \underline{\underline{d}}_{1} \underline{e}_{1}\right\}^{-}}$ & {$[1,0,0,2,0]$} & 1 & 2 & 1 & 3 & 2 & 3 & 4 \\
\hline $\mathcal{V}_{\left\{\underline{a}_{1} \underline{b}_{1} \underline{c}_{1}\right\}}$ & {$[0,0,1,0,0]$} & 1 & 1 & 1 & 2 & 1 & 2 & 2 \\
\hline
\end{tabular}

Table 3. Contributions of Each Type-I Superfield to the component bosonic and fermionic representation content of the $10 \mathrm{D}, \mathcal{N}=1$ supergravity multiplet at Level-16/17 of Type-IIB Scalar Superfield Decomposition.

Next in this line of reasoning, we may consider a truncation of $10 \mathrm{D}, \mathcal{N}=$ IIA superspace to $10 \mathrm{D}, \mathcal{N}=1$ superspace. As our discussions in this section showed, there are choices to explore as the putative supergravity prepotential. The ADA algorithms inform us there are only a few options to explore, at least under the set of assumptions we are using. These include the ten possibilities given by $\mathcal{V}_{\left\{\underline{a}_{1} \underline{a}_{2} \underline{a}_{3}\right\}}, \mathcal{V}_{\left\{\underline{a}_{1} \underline{b}_{1}, \underline{a}_{2} \underline{b}_{2}\right\}}, \mathcal{V}_{\left\{\underline{a}_{1}, \underline{a}_{2}, \underline{a}_{3}, \underline{a}_{4}\right\}}, \mathcal{V}_{\left\{\underline{a}_{2}, \underline{a}_{3} \mid \underline{a}_{1} \underline{b}_{1} \underline{c}_{1}\right\}}$,

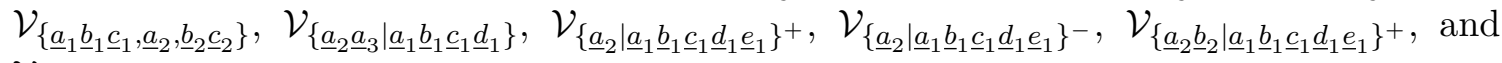
$\mathcal{V}_{\left\{\underline{a}_{2} \underline{b}_{2} \mid \underline{a}_{1} \underline{b}_{1} \underline{c}_{1} \underline{d}_{1} \underline{e}_{1}\right\}^{-}}$.

However, among the ten choices indicated by the two tables in this section, there is one that is extremely interesting as it contains a single copy of each of the component irreps that were the parameters of our ADA scan. This representation is the superfield $\mathcal{V}_{\left\{\underline{a}_{1}, \underline{a}_{2}, \underline{a}_{3}, \underline{a}_{4}\right\}}$, with Dynkin Label $[4,0,0,0,0]$ which corresponds to a $10 \mathrm{D}$, totally symmetical, completely traceless fourth rank tensor superfield

$$
\mathcal{V}_{[4,0,0,0,0]}\left(x^{\underline{a}}, \theta^{\alpha}\right)=\mathcal{V}_{\left\{\underline{a}_{1}, \underline{a}_{2}, \underline{a}_{3}, \underline{a}_{4}\right\}}\left(x^{\underline{a}}, \theta^{\alpha}\right) \text {. }
$$

It also possesses the property that the conformal graviton irrep occurs in the middle level of the superfield. These two properties suggest to us this should be the superfield that is the most likely candidate for the $10 \mathrm{D}, \mathcal{N}=1$ supergravity prepotential.

The next task becomes one of finding if there are Type-I superfields (SF's) in the expansions shown in (5.1) and (5.2) that will allow the patterns of height assignments 


\begin{tabular}{|c|c|c|c|c|c|c|c|c|}
\hline Type-I SF & Dynkin Label & $b_{\{10\}}$ & $b_{\{120\}}$ & $b_{\{16\}}$ & $b_{\{144\}}$ & $\theta$-Level & $\begin{array}{c}\text { Boson } \\
\text { Height }\end{array}$ & $\begin{array}{c}\text { Fermion } \\
\text { Height }\end{array}$ \\
\hline$\Psi_{\left\{\underline{a}_{1}, \underline{a}_{2}, \underline{a}_{3}\right\} \alpha}$ & {$[3,0,0,1,0]$} & 1 & 3 & 2 & 3 & 9 & 7 & 8 \\
\hline$\Psi_{\left\{\underline{a}_{2} \mid \underline{a}_{1} \underline{\underline{b}}_{1} \underline{\underline{c}}_{1}\right\}^{\alpha}}$ & {$[1,0,1,0,1]$} & 1 & 4 & 2 & 5 & 9 & 7 & 8 \\
\hline
\end{tabular}

Table 4. Type-I Superfield Contributions to Bosons at Level-16 \& Fermions at Level-17 in TypeIIA Scalar Superfield Decomposition.

\begin{tabular}{|c|c|c|c|c|c|c|c|c|c|}
\hline Type-I SF & Dynkin Label & $b_{\{1\}}$ & $b_{\{45\}}$ & $b_{\{210\}}$ & $b_{\{\overline{16}\}}$ & $b_{\{\overline{144}\}}$ & $\theta$-Level & $\begin{array}{c}\text { Boson } \\
\text { Height }\end{array}$ & $\begin{array}{c}\text { Fermion } \\
\text { Height }\end{array}$ \\
\hline$\Psi_{\left\{\underline{a}_{1}, \underline{a}_{2}, \underline{a}_{3}\right\}}{ }^{\alpha}$ & {$[3,0,0,0,1]$} & 1 & 2 & 3 & 2 & 3 & 9 & 7 & 8 \\
\hline$\Psi_{\left\{\underline{a}_{2} \mid \underline{a}_{1} \underline{\underline{c}}_{1} \underline{c}_{1}\right\}^{\alpha}}$ & {$[1,0,1,1,0]$} & 1 & 3 & 6 & 2 & 5 & 9 & 7 & 8 \\
\hline
\end{tabular}

Table 5. Type-I Superfield Contributions to Bosons at Level-16 \& Fermions at Level-17 in Type-IIB Scalar Superfield Decomposition.

and irreps seen in (4.6) and (4.7) to be found within them? So we set up an ADA scan in (5.1) looking for the first pattern and one in (5.2) looking for the second pattern at levels compatible with the $10 \mathrm{D}, \mathcal{N}=1 \mathrm{SG}$ prepotential identified in (5.3). The task is simplified by an observation on how the non-manifest supercharges act relative to the identification of the $[4,0,0,0,0]$ supergravity prepotential which occurs at $\theta$-Level eight. The second nonmanifest supercharge must connect quantities at different $\theta$-Levels. The simplest example of this phenomenon is seen in the $4 \mathrm{D}, \mathcal{N}=1$ chiral supermultiplet. The spin- 0 fields are at the lowest $\theta$-Level and a supercharge acting on these spin- 0 fields connect them to the spin- $1 / 2$ fields at one $\theta$-Level higher. Translating this lesson for the present consideration, the $[4,0,0,0,0]$ supergravity prepotential should be connected to a $10 \mathrm{D}, \mathcal{N}=1$ superfield at $\theta$-Level nine. So the present scan can be restricted to a search at this level. The results of these scans are shown in tables 4 and 5 .

Beyond this point, ADA scans provide no guidance or other means will be required for further progress. But these scans have provided valuable insights that have "shone a spotlight" within these systems containing up to 2,147,483,648 bosonic degrees of freedom and 2,147,483,648 fermionic degrees of freedom. The results derived indicate where future investigations might show the greatest return on investment of time and energy.

\section{Conclusion}

In sections 2 and 3 , we have demonstrated the prepotential basis for the argument that imposing a sufficient number of constraints on the superframe fields to express them in terms of $\Psi$ and $\mathrm{H}_{\alpha} \underline{a}$ as their only independent superfield variables necessarily leads to Poincaré systems that nevertheless possess Weyl covariance. This observation was used to derive a set of infinitesimal Weyl scaling laws for ten and eleven supergravity. On this basis the transformation laws for the superspace torsion and curvature supertensors 
were derived and the super Weyl covariant supertensors containing the on-shell degrees of freedom were thus identified within components of the torsion supertensors. In the case of the $\mathcal{N}=1$ theories in 10D and 11D, the infinitesimal Weyl scaling laws confirm results derived previously in the work of [7] and [16]. For the cases $10 \mathrm{D}, \mathcal{N}=2 \mathrm{~A}$ and $\mathcal{N}=2 \mathrm{~B}$, the infinitesimal Weyl scaling laws derived in section 3 have not been presented previously. In section 4, the conceptual basis provided by the concept of "adynkras" [23, 24] was reviewed in a way that is relevant to the discussion of supergravity theories in ten and eleven dimensions. Finally, in section 5, the presentations and results of the previous sections were used to identify possible supergravity prepotentials candidates.

In the discussion in [24] of the $11 \mathrm{D}, \mathcal{N}=1$ theory, the primary putative supergravity superfield was a scalar superfield also. To distinguish it from the superfields discussed in (5.1) and (5.2), we can denote the eleven dimensional scalar superfield by $\mathcal{V}_{11}\left(x^{\underline{a}}, \theta^{\alpha}\right)$ which together with $\mathcal{V}_{\text {IIA }}\left(x^{x}, \theta^{\alpha}, \theta^{\dot{\alpha}}\right)$ and $\mathcal{V}_{\text {IIB }}\left(x^{x}, \theta^{\alpha}, \theta^{\alpha}\right),{ }^{10}$ form a triplet of superfields that hold the possibility of being the fundamental supergravity prepotentials of $11 \mathrm{D}, \mathcal{N}=1$, $10 \mathrm{D}, \mathcal{N}=$ IIA, and $10 \mathrm{D}, \mathcal{N}=$ IIB theories. This raises the intriguing possibility this triplet is related to duality properties among these high dimensional models.

"The best way to have a good idea is to have a lot of ideas".

Linus Pauling

\section{Acknowledgments}

We wish to acknowledge discussions with B. de Wit, S.J. Gates III, S. Kuzenko, W. Linch, W. Siegel, M. Roček, and E. Witten. The research of S.J.G., Y. Hu, and S.-N. Mak is supported in part by the endowment of the Ford Foundation Professorship of Physics at Brown University and they gratefully acknowledge the support of the Brown Theoretical Physics Center.

\section{A 10D Weyl scaling properties of weight $\geq \frac{3}{2}$ super tensors}

In this appendix, we record the Weyl scaling transformation laws for the super torsion and super curvature tensors of scale weight equal or greater than three-halves in the respective cases of the $\mathcal{N}=2 \mathrm{~A}, \mathcal{N}=2 \mathrm{~B}$ and $\mathcal{N}=1$ theories.

\section{A.1 $10 \mathrm{D}, \mathcal{N}=2 \mathrm{~A}$}

$$
\begin{aligned}
\delta_{S} T_{\underline{a} b}{ }^{\gamma}= & \frac{3}{2} L T_{\underline{a} b}^{\gamma}+i \frac{1}{5}\left(\sigma_{[\underline{a}}\right)^{\alpha \gamma}\left(\nabla_{\underline{b}]} \nabla_{\alpha} L\right)-i \frac{1}{5}\left(\sigma_{[\underline{a}}\right)^{\alpha \beta}\left(\nabla_{\alpha} L\right) T_{\beta \mid \underline{b}]}{ }^{\gamma} \\
& -i \frac{1}{5}\left(\sigma_{[\underline{a}}\right)^{\dot{\alpha} \dot{\beta}}\left(\nabla_{\dot{\alpha}} L\right) T_{\dot{\beta} \mid \underline{b}]} \gamma+i \frac{1}{5}\left(\sigma_{\underline{c}}\right)^{\gamma \delta}\left(\nabla_{\delta} L\right) T_{\underline{a} b} \underline{c}
\end{aligned}
$$

\footnotetext{
${ }^{10}$ Of course, the range of the spinor index for the $11 \mathrm{D}$ theory goes from one to thirty-two while the range for the $10 \mathrm{D}$ theories is half of this.
} 


$$
\begin{aligned}
& \delta_{S} T_{\underline{a} b} \dot{\gamma}=\frac{3}{2} L T_{\underline{a}} \dot{\gamma}+i \frac{1}{5}\left(\sigma_{[\underline{a}}\right)^{\dot{\alpha} \dot{\gamma}}\left(\nabla_{\underline{b}]} \nabla_{\dot{\alpha}} L\right)-i \frac{1}{5}\left(\sigma_{[\underline{a}}\right)^{\alpha \beta}\left(\nabla_{\alpha} L\right) T_{\beta \mid \underline{b}]} \dot{\gamma} \\
& -i \frac{1}{5}\left(\sigma_{[\underline{a}}\right)^{\dot{\alpha} \dot{\beta}}\left(\nabla_{\dot{\alpha}} L\right) T_{\dot{\beta} \mid \underline{b}]} \dot{\gamma}+i \frac{1}{5}\left(\sigma_{\underline{c}}\right)^{\dot{\gamma} \dot{\delta}}\left(\nabla_{\dot{\delta}} L\right) T_{\underline{a}} b^{\underline{c}}, \\
& \delta_{S} R_{\alpha \underline{b}} \underline{\underline{d} e}=\frac{3}{2} L R_{\alpha \underline{\underline{b}}} \underline{\underline{d} e}-T_{\alpha \underline{\underline{b}}} \underline{[\underline{d}}\left(\nabla^{e} L\right)+\frac{1}{5} T_{\alpha \underline{\underline{b}}}{ }^{\gamma}\left(\sigma^{\underline{d} e}\right)_{\gamma}{ }^{\delta}\left(\nabla_{\delta} L\right)+\frac{1}{5} T_{\alpha \underline{b}} \dot{\dot{\gamma}}\left(\sigma^{\underline{d} e}\right)_{\dot{\gamma}}^{\dot{\delta}}\left(\nabla_{\dot{\delta}} L\right) \\
& +\frac{1}{5}\left(\sigma^{-}\right)_{\alpha}{ }_{\alpha}^{\beta}\left(\nabla_{\underline{b}} \nabla_{\beta} L\right)+i \frac{1}{5}\left(\sigma_{\underline{b}}\right)^{\delta \beta}\left(\nabla_{\delta} L\right) R_{\alpha \beta} \underline{d} e+i \frac{1}{5}\left(\sigma_{\underline{b}}\right)^{\dot{\delta} \dot{\beta}}\left(\nabla_{\dot{\delta}} L\right) R_{\alpha \dot{\beta}} \underline{\underline{d} e} \\
& -\left(\nabla_{\alpha} \nabla^{[\underline{d}} L\right) \delta_{\underline{b}} \underline{e}, \\
& \left.\delta_{S} R_{\dot{\alpha} \underline{b}} \underline{\underline{d} e}=\frac{3}{2} L R_{\dot{\alpha} \underline{b}} \underline{\underline{d}} \underline{e}-T_{\dot{\alpha} \underline{b}} \underline{[d}\left(\nabla^{e}\right] L\right)+\frac{1}{5} T_{\dot{\alpha} \underline{\underline{\gamma}}}^{\gamma}\left(\sigma^{\underline{d} e}\right)_{\gamma}{ }^{\delta}\left(\nabla_{\delta} L\right)+\frac{1}{5} T_{\dot{\alpha} \underline{b}} \dot{\dot{\gamma}}\left(\sigma^{\underline{d} e}\right)_{\dot{\gamma}}^{\dot{\delta}}\left(\nabla_{\dot{\delta}} L\right) \\
& +\frac{1}{5}\left(\sigma^{\underline{d} e}\right)_{\dot{\alpha}}^{\dot{\beta}}\left(\nabla_{\underline{b}} \nabla_{\dot{\beta}} L\right)+i \frac{1}{5}\left(\sigma_{\underline{b}}\right)^{\delta \beta}\left(\nabla_{\delta} L\right) R_{\dot{\alpha} \beta^{\beta}} \underline{d e}+i \frac{1}{5}\left(\sigma_{\underline{b}}\right)^{\dot{\delta} \dot{\beta}}\left(\nabla_{\dot{\delta}} L\right) R_{\dot{\alpha} \dot{\beta}} \underline{d e} \\
& -\left(\nabla_{\dot{\alpha}} \nabla^{[\underline{d}} L\right) \delta_{\underline{b}} \underline{e} \text {, } \\
& \delta_{S} R_{\underline{a}} b^{\underline{\underline{d}} e}=2 L R_{\underline{a}} b^{\underline{d} e}-T_{\underline{a}} b^{[\underline{d}}\left(\nabla^{\underline{e}]} L\right)+\frac{1}{5} T_{\underline{a} b^{\gamma}}\left(\sigma^{\underline{d} e}\right)_{\gamma}^{\delta}\left(\nabla_{\delta} L\right)+\frac{1}{5} T_{\underline{a}} b^{\dot{\gamma}}\left(\sigma^{\underline{d} e}\right) \dot{\gamma}^{\dot{\delta}}\left(\nabla_{\dot{\delta}} L\right)
\end{aligned}
$$

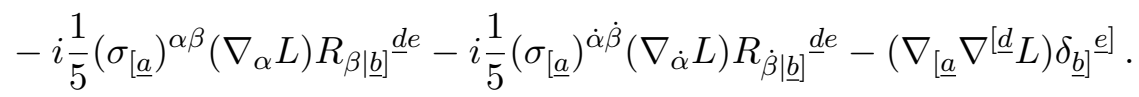

\section{A.2 10D, $\mathcal{N}=2 \mathrm{~B}$}

$$
\begin{aligned}
& \delta_{S} T_{\underline{a} b^{\gamma}}{ }^{\gamma}=\left(\frac{1}{2} L+\bar{L}\right) T_{\underline{a} b}{ }^{\gamma}+i\left(\sigma_{[\underline{a}}\right)^{\alpha \gamma}\left(\nabla_{\underline{b}]} \bar{\nabla}_{\alpha}\left(\frac{1}{32} L+\frac{27}{160} \bar{L}\right)\right)-i\left(\sigma_{[\underline{a}}\right)^{\alpha \beta}\left(\bar{\nabla}_{\alpha}\left(\frac{1}{32} L+\frac{27}{160} \bar{L}\right)\right) T_{\beta \mid \underline{b}]^{\gamma}} \\
& -i\left(\sigma_{[\underline{a}}\right)^{\alpha \beta}\left(\nabla_{\alpha}\left(\frac{1}{32} \bar{L}+\frac{27}{160} L\right)\right) T_{\bar{\beta} \mid \underline{b}]}^{\gamma}+i\left(\sigma_{\underline{c}}\right)^{\gamma \delta}\left(\bar{\nabla}_{\delta}\left(\frac{1}{32} L+\frac{27}{160} \bar{L}\right)\right) T_{\underline{a}} b^{\underline{c}}, \\
& \delta_{S} T_{\underline{a}} b^{\bar{\gamma}}=\left(\frac{1}{2} \bar{L}+L\right) T_{\underline{a} b} \bar{\gamma}^{\bar{\gamma}}+i\left(\sigma_{[\underline{a}}\right)^{\alpha \gamma}\left(\nabla_{\underline{b}]} \nabla_{\alpha}\left(\frac{1}{32} \bar{L}+\frac{27}{160} L\right)\right)-i\left(\sigma_{[\underline{a}}\right)^{\alpha \beta}\left(\bar{\nabla}_{\alpha}\left(\frac{1}{32} L+\frac{27}{160} \bar{L}\right)\right) T_{\beta \mid \underline{b}]^{\bar{\gamma}}} \\
& -i\left(\sigma_{[\underline{a}}\right)^{\alpha \beta}\left(\nabla_{\alpha}\left(\frac{1}{32} \bar{L}+\frac{27}{160} L\right)\right) T_{\bar{\beta} \mid \underline{b}]}{ }^{\bar{\gamma}}+i\left(\sigma_{\underline{c}}\right)^{\gamma \delta}\left(\nabla_{\delta}\left(\frac{1}{32} \bar{L}+\frac{27}{160} L\right)\right) T_{\underline{a}} b^{\underline{c}} . \\
& \delta_{S} R_{\alpha \underline{\underline{b}}} \underline{\underline{d} e}=\left(\frac{1}{2} \bar{L}+L\right) R_{\alpha \underline{\underline{b}}} \underline{\underline{d} e}-\frac{1}{2} T_{\alpha \underline{\underline{b}}} \underline{[\underline{d}}\left(\nabla^{e}(L+\bar{L})\right)+\frac{1}{5} T_{\alpha \underline{\underline{b}}}{ }^{\gamma}\left(\sigma^{\underline{d} e}\right)_{\gamma}{ }^{\delta}\left(\nabla_{\delta} L\right)+\frac{1}{5} T_{\alpha \underline{\underline{b}}}{ }^{\bar{\gamma}}\left(\sigma^{\underline{\underline{d}} e}\right)_{\gamma}{ }^{\delta}\left(\bar{\nabla}_{\delta} \bar{L}\right) \\
& +\frac{1}{5}\left(\sigma^{\underline{d} e}\right)_{\alpha}^{\beta}\left(\nabla_{\underline{b}} \nabla_{\beta} L\right)+i\left(\sigma_{\underline{b}}\right)^{\delta \beta}\left(\bar{\nabla}_{\delta}\left(\frac{1}{32} L+\frac{27}{160} \bar{L}\right)\right) R_{\alpha \beta^{\underline{d}}}{ }^{e} \\
& +i\left(\sigma_{\underline{b}}\right)^{\delta \beta}\left(\nabla_{\delta}\left(\frac{1}{32} \bar{L}+\frac{27}{160} L\right)\right) R_{\alpha \bar{\beta}^{\underline{d}}}-\frac{1}{2}\left(\nabla_{\alpha} \nabla^{[\underline{d}}(L+\bar{L})\right) \delta_{\underline{b}}^{e},
\end{aligned}
$$

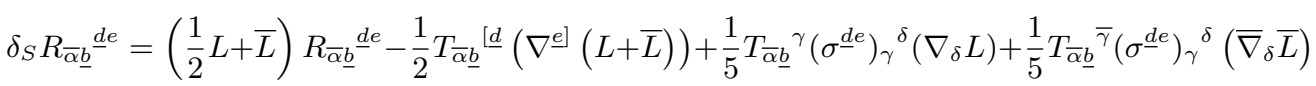

$$
\begin{aligned}
& +\frac{1}{5}\left(\sigma^{\underline{d} e}\right)_{\alpha}^{\beta}\left(\nabla_{\underline{b}} \bar{\nabla}_{\beta} \bar{L}\right)+i\left(\sigma_{\underline{b}}\right)^{\delta \beta}\left(\bar{\nabla}_{\delta}\left(\frac{1}{32} L+\frac{27}{160} \bar{L}\right)\right) R_{\bar{\alpha} \beta^{\underline{d}}}+i\left(\sigma_{\underline{b}}\right)^{\delta \beta}\left(\nabla_{\delta}\left(\frac{1}{32} \bar{L}+\frac{27}{160} L\right)\right) R_{\bar{\alpha} \bar{\beta}^{\underline{d}} \underline{e}} \\
& -\frac{1}{2}\left(\bar{\nabla}_{\alpha} \nabla^{[\underline{d}}(L+\bar{L})\right) \delta_{\underline{b}}^{e} \text {, }
\end{aligned}
$$

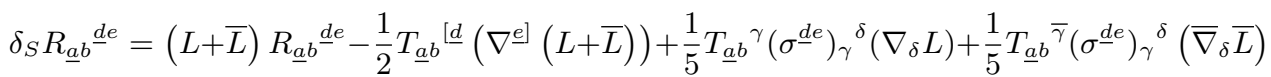

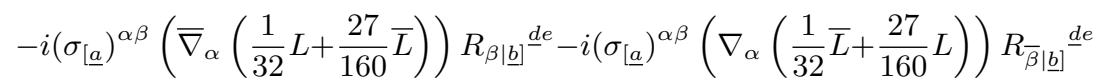

$$
\begin{aligned}
& -\frac{1}{2}\left(\nabla_{[\underline{[}} \nabla^{[\underline{d}}(L+\bar{L})\right) \delta_{\underline{b}]}^{e]} \text {. }
\end{aligned}
$$




\section{A.3 10D, $\mathcal{N}=1$}

$$
\begin{aligned}
& \delta_{S} T_{\underline{a} b}{ }^{\gamma}=\frac{3}{2} L T_{\underline{a} b}{ }^{\gamma}+i \frac{2}{5}\left(\sigma_{[\underline{a}}\right)^{\alpha \gamma}\left(\nabla_{\underline{b}]} \nabla_{\alpha} L\right)-i \frac{2}{5}\left(\sigma_{[\underline{a}}\right)^{\alpha \beta}\left(\nabla_{\alpha} L\right) T_{\beta \mid \underline{b}]}^{\gamma} \\
& +i \frac{2}{5}\left(\sigma_{\underline{c}}\right)^{\gamma \delta}\left(\nabla_{\delta} L\right) T_{\underline{a}} b^{\underline{c}}, \\
& \delta_{S} R_{\alpha \underline{b}} \underline{\underline{d} e}=\frac{3}{2} L R_{\alpha \underline{b}} \underline{\underline{d} e}-T_{\alpha \underline{b}} \underline{[d}^{\underline{d}}\left(\nabla^{e} L\right)+\frac{1}{5} T_{\alpha \underline{b}}^{\gamma}\left(\sigma^{\underline{d} e}\right)_{\gamma}{ }^{\delta}\left(\nabla_{\delta} L\right) \\
& \left.+\frac{1}{5}\left(\sigma^{\underline{d} e}\right)_{\alpha}^{\beta}\left(\nabla_{\underline{b}} \nabla_{\beta} L\right)+i \frac{2}{5}\left(\sigma_{\underline{b}}\right)^{\delta \beta}\left(\nabla_{\delta} L\right) R_{\alpha \beta^{\underline{d}}} e-\left(\nabla_{\alpha} \nabla^{[\underline{d}} L\right) \delta_{\underline{b}} \underline{e}\right] \\
& \delta_{S} R_{\underline{a}} b^{\underline{d} e}=2 L R_{\underline{a}} b^{\underline{d} e}-T_{\underline{a} b} \underline{[d}\left(\nabla^{e} L\right)+\frac{1}{5} T_{\underline{a} b^{\gamma}}\left(\sigma^{\underline{d} e}\right)_{\gamma}^{\delta}\left(\nabla_{\delta} L\right) \\
& -i \frac{2}{5}\left(\sigma_{[\underline{a}}\right)^{\alpha \beta}\left(\nabla_{\alpha} L\right) R_{\beta \mid \underline{b}]^{d}}-\left(\nabla_{[\underline{a}} \nabla^{[\underline{d}} L\right) \delta_{\left.\underline{b}]^{e}\right]} .
\end{aligned}
$$

\section{B Adynkra libraries}

In this appendix, we explicitly present the adynkra libraries of all superfields that appear in equation (4.4) in the text.

\section{B.1 Dynkin Label library of $\mathcal{V}_{[0,0,1,0,0]}$}

- Level-0: $[0,0,1,0,0]$

- Level-1: $[0,0,0,0,1] \oplus[1,0,0,1,0] \oplus[0,1,0,0,1] \oplus[0,0,1,1,0]$

- Level-2: $[0,0,0,0,0] \oplus[0,1,0,0,0] \oplus[2,0,0,0,0] \oplus(2)[0,0,0,1,1] \oplus[0,2,0,0,0] \oplus$ $[1,0,1,0,0] \oplus[1,0,0,2,0] \oplus[1,0,0,0,2] \oplus[0,0,2,0,0] \oplus[0,1,0,1,1]$

- Level-3: $[0,0,0,1,0] \oplus(2)[1,0,0,0,1] \oplus(2)[0,1,0,1,0] \oplus[0,0,0,0,3] \oplus[2,0,0,1,0] \oplus$ $(2)[0,0,1,0,1] \oplus[0,0,0,2,1] \oplus(2)[1,1,0,0,1] \oplus[0,2,0,1,0] \oplus[1,0,1,1,0] \oplus[1,0,0,1,2] \oplus$ $[0,1,1,0,1]$

- Level-4: $\quad(2)[0,0,1,0,0] \oplus[0,0,0,0,2] \oplus(2)[1,1,0,0,0] \oplus(3)[1,0,0,1,1] \oplus$ $(2)[0,1,1,0,0] \oplus[0,1,0,2,0] \oplus(3)[0,1,0,0,2] \oplus(2)[2,0,1,0,0] \oplus[1,2,0,0,0] \oplus$ $[2,0,0,0,2] \oplus[0,0,0,1,3] \oplus[0,0,1,1,1] \oplus[0,2,1,0,0] \oplus(2)[1,1,0,1,1] \oplus[1,0,1,0,2]$

- Level-5: $[1,0,0,1,0] \oplus(3)[0,1,0,0,1] \oplus(2)[2,0,0,0,1] \oplus[0,0,1,1,0] \oplus(2)[0,0,0,1,2] \oplus$ $[3,0,0,1,0] \oplus(3)[1,1,0,1,0] \oplus(2)[1,0,0,0,3] \oplus(2)[0,2,0,0,1] \oplus(4)[1,0,1,0,1] \oplus$ $[1,0,0,2,1] \oplus(2)[2,1,0,0,1] \oplus[0,1,1,1,0] \oplus[0,0,1,0,3] \oplus(2)[0,1,0,1,2] \oplus[2,0,1,1,0] \oplus$ $[1,2,0,1,0] \oplus[2,0,0,1,2] \oplus[1,1,1,0,1]$

- Level-6: $[2,0,0,0,0] \oplus[0,0,0,1,1] \oplus[4,0,0,0,0] \oplus(2)[0,2,0,0,0] \oplus(2)[1,0,1,0,0] \oplus$ $[1,0,0,2,0] \oplus(3)[1,0,0,0,2] \oplus(2)[2,1,0,0,0] \oplus[0,0,0,0,4] \oplus(2)[0,0,2,0,0] \oplus$ $(3)[0,1,0,1,1] \oplus(2)[0,0,1,0,2] \oplus(4)[2,0,0,1,1] \oplus[0,0,0,2,2] \oplus[3,0,1,0,0] \oplus$ $[2,2,0,0,0] \oplus[3,0,0,2,0] \oplus[3,0,0,0,2] \oplus(3)[1,1,1,0,0] \oplus[1,1,0,2,0] \oplus(3)[1,1,0,0,2] \oplus$ $[1,0,0,1,3] \oplus(2)[1,0,1,1,1] \oplus[0,2,0,1,1] \oplus[2,0,2,0,0] \oplus[0,1,1,0,2] \oplus[2,1,0,1,1]$ 
- Level-7: $[1,0,0,0,1] \oplus[0,1,0,1,0] \oplus[0,0,0,0,3] \oplus(2)[2,0,0,1,0] \oplus(2)[0,0,1,0,1] \oplus$ $[0,0,0,2,1] \oplus(3)[3,0,0,0,1] \oplus(4)[1,1,0,0,1] \oplus[4,0,0,1,0] \oplus[0,2,0,1,0] \oplus$ $(4)[1,0,1,1,0] \oplus(3)[1,0,0,1,2] \oplus(3)[2,1,0,1,0] \oplus[0,1,0,0,3] \oplus[2,0,0,0,3] \oplus$ (3) $[0,1,1,0,1] \oplus[0,0,2,1,0] \oplus[0,1,0,2,1] \oplus(3)[2,0,1,0,1] \oplus[1,2,0,0,1] \oplus[3,1,0,0,1] \oplus$ $(2)[2,0,0,2,1] \oplus[0,0,1,1,2] \oplus[3,0,1,1,0] \oplus[1,1,1,1,0] \oplus[1,0,2,0,1] \oplus[1,1,0,1,2]$

- Level-8: $[0,0,1,0,0] \oplus[3,0,0,0,0] \oplus[1,1,0,0,0] \oplus(3)[1,0,0,1,1] \oplus(2)[0,1,1,0,0] \oplus$ $(2)[0,1,0,2,0] \oplus(2)[0,1,0,0,2] \oplus(5)[2,0,1,0,0] \oplus[1,2,0,0,0] \oplus(2)[3,1,0,0,0] \oplus$ $(2)[2,0,0,2,0] \oplus(2)[2,0,0,0,2] \oplus(3)[0,0,1,1,1] \oplus(2)[1,0,2,0,0] \oplus(3)[3,0,0,1,1] \oplus$ $[0,2,1,0,0] \oplus(4)[1,1,0,1,1] \oplus[4,0,1,0,0] \oplus(2)[1,0,1,2,0] \oplus(2)[1,0,1,0,2] \oplus$ $[1,0,0,2,2] \oplus[2,1,1,0,0] \oplus[0,0,3,0,0] \oplus[2,1,0,2,0] \oplus[2,1,0,0,2] \oplus[0,1,1,1,1] \oplus$ $[2,0,1,1,1]$

- Level-9: $[1,0,0,1,0] \oplus[0,1,0,0,1] \oplus[0,0,0,3,0] \oplus(2)[2,0,0,0,1] \oplus(2)[0,0,1,1,0] \oplus$ $[0,0,0,1,2] \oplus(3)[3,0,0,1,0] \oplus(4)[1,1,0,1,0] \oplus[4,0,0,0,1] \oplus[0,2,0,0,1] \oplus$ $(4)[1,0,1,0,1] \oplus(3)[1,0,0,2,1] \oplus(3)[2,1,0,0,1] \oplus[0,1,0,3,0] \oplus[2,0,0,3,0] \oplus$ $(3)[0,1,1,1,0] \oplus[0,0,2,0,1] \oplus[0,1,0,1,2] \oplus(3)[2,0,1,1,0] \oplus[1,2,0,1,0] \oplus[3,1,0,1,0] \oplus$ $(2)[2,0,0,1,2] \oplus[0,0,1,2,1] \oplus[3,0,1,0,1] \oplus[1,1,1,0,1] \oplus[1,0,2,1,0] \oplus[1,1,0,2,1]$

- Level-10: $[2,0,0,0,0] \oplus[0,0,0,1,1] \oplus[4,0,0,0,0] \oplus(2)[0,2,0,0,0] \oplus(2)[1,0,1,0,0] \oplus$ $(3)[1,0,0,2,0] \oplus[1,0,0,0,2] \oplus(2)[2,1,0,0,0] \oplus[0,0,0,4,0] \oplus(2)[0,0,2,0,0] \oplus$ $(3)[0,1,0,1,1] \oplus(2)[0,0,1,2,0] \oplus(4)[2,0,0,1,1] \oplus[0,0,0,2,2] \oplus[3,0,1,0,0] \oplus$ $[2,2,0,0,0] \oplus[3,0,0,2,0] \oplus[3,0,0,0,2] \oplus(3)[1,1,1,0,0] \oplus(3)[1,1,0,2,0] \oplus[1,1,0,0,2] \oplus$ $[1,0,0,3,1] \oplus(2)[1,0,1,1,1] \oplus[0,2,0,1,1] \oplus[2,0,2,0,0] \oplus[0,1,1,2,0] \oplus[2,1,0,1,1]$

- Level-11: $[1,0,0,0,1] \oplus(3)[0,1,0,1,0] \oplus(2)[2,0,0,1,0] \oplus[0,0,1,0,1] \oplus(2)[0,0,0,2,1] \oplus$ $[3,0,0,0,1] \oplus(3)[1,1,0,0,1] \oplus(2)[1,0,0,3,0] \oplus(2)[0,2,0,1,0] \oplus(4)[1,0,1,1,0] \oplus$ $[1,0,0,1,2] \oplus(2)[2,1,0,1,0] \oplus[0,1,1,0,1] \oplus[0,0,1,3,0] \oplus(2)[0,1,0,2,1] \oplus[2,0,1,0,1] \oplus$ $[1,2,0,0,1] \oplus[2,0,0,2,1] \oplus[1,1,1,1,0]$

- Level-12: $\quad(2)[0,0,1,0,0] \oplus[0,0,0,2,0] \oplus(2)[1,1,0,0,0] \oplus(3)[1,0,0,1,1] \oplus$ $(2)[0,1,1,0,0] \oplus(3)[0,1,0,2,0] \oplus[0,1,0,0,2] \oplus(2)[2,0,1,0,0] \oplus[1,2,0,0,0] \oplus$ $[2,0,0,2,0] \oplus[0,0,0,3,1] \oplus[0,0,1,1,1] \oplus[0,2,1,0,0] \oplus(2)[1,1,0,1,1] \oplus[1,0,1,2,0]$

- Level-13: $[0,0,0,0,1] \oplus(2)[1,0,0,1,0] \oplus(2)[0,1,0,0,1] \oplus[0,0,0,3,0] \oplus[2,0,0,0,1] \oplus$ $(2)[0,0,1,1,0] \oplus[0,0,0,1,2] \oplus(2)[1,1,0,1,0] \oplus[0,2,0,0,1] \oplus[1,0,1,0,1] \oplus[1,0,0,2,1] \oplus$ $[0,1,1,1,0]$

- Level-14: $[0,0,0,0,0] \oplus[0,1,0,0,0] \oplus[2,0,0,0,0] \oplus(2)[0,0,0,1,1] \oplus[0,2,0,0,0] \oplus$ $[1,0,1,0,0] \oplus[1,0,0,2,0] \oplus[1,0,0,0,2] \oplus[0,0,2,0,0] \oplus[0,1,0,1,1]$

- Level-15: $[0,0,0,1,0] \oplus[1,0,0,0,1] \oplus[0,1,0,1,0] \oplus[0,0,1,0,1]$

- Level-16: $[0,0,1,0,0]$ 


\section{B.2 Dynkin Label library of $\mathcal{V}_{[1,0,1,0,1]}$}

- Level-0: $[1,0,1,0,1]$

- Level-1: $\quad[1,0,1,0,0] \oplus[1,0,0,0,2] \oplus[0,0,2,0,0] \oplus[0,1,0,1,1] \oplus[0,0,1,0,2] \oplus$ $[2,0,0,1,1] \oplus[1,1,1,0,0] \oplus[1,1,0,0,2] \oplus[1,0,1,1,1]$

- Level-2: $[1,0,0,0,1] \oplus[0,1,0,1,0] \oplus[0,0,0,0,3] \oplus[2,0,0,1,0] \oplus(2)[0,0,1,0,1] \oplus$ $[0,0,0,2,1] \oplus[3,0,0,0,1] \oplus(3)[1,1,0,0,1] \oplus[0,2,0,1,0] \oplus(3)[1,0,1,1,0] \oplus$ $(3)[1,0,0,1,2] \oplus[2,1,0,1,0] \oplus[0,1,0,0,3] \oplus[2,0,0,0,3] \oplus(3)[0,1,1,0,1] \oplus[0,0,2,1,0] \oplus$ $[0,1,0,2,1] \oplus(2)[2,0,1,0,1] \oplus[1,2,0,0,1] \oplus[2,0,0,2,1] \oplus[0,0,1,1,2] \oplus[1,1,1,1,0] \oplus$ $[1,0,2,0,1] \oplus[1,1,0,1,2]$

- Level-3: $\quad[0,0,1,0,0] \oplus[0,0,0,2,0] \oplus[0,0,0,0,2] \oplus[3,0,0,0,0] \oplus(2)[1,1,0,0,0] \oplus$ $(5)[1,0,0,1,1] \oplus(4)[0,1,1,0,0] \oplus(3)[0,1,0,2,0] \oplus(4)[0,1,0,0,2] \oplus(4)[2,0,1,0,0] \oplus$ $(2)[1,2,0,0,0] \oplus[3,1,0,0,0] \oplus(2)[2,0,0,2,0] \oplus(4)[2,0,0,0,2] \oplus[0,0,0,3,1] \oplus$ $(2)[0,0,0,1,3] \oplus(5)[0,0,1,1,1] \oplus[1,0,0,0,4] \oplus(4)[1,0,2,0,0] \oplus(2)[3,0,0,1,1] \oplus$ $(2)[0,2,1,0,0] \oplus(7)[1,1,0,1,1] \oplus[0,2,0,2,0] \oplus(2)[0,2,0,0,2] \oplus(2)[1,0,1,2,0] \oplus$ $(5)[1,0,1,0,2] \oplus(2)[1,0,0,2,2] \oplus(2)[2,1,1,0,0] \oplus[0,0,3,0,0] \oplus[2,1,0,2,0] \oplus$ $(2)[2,1,0,0,2] \oplus[0,0,2,0,2] \oplus[0,1,0,1,3] \oplus(3)[0,1,1,1,1] \oplus[2,0,0,1,3] \oplus$ (2) $[2,0,1,1,1] \oplus[1,1,2,0,0] \oplus[1,2,0,1,1] \oplus[1,1,1,0,2]$

- Level-4: $\quad(2)[1,0,0,1,0] \oplus(4)[0,1,0,0,1] \oplus(2)[0,0,0,3,0] \oplus(4)[2,0,0,0,1] \oplus$ $(5)[0,0,1,1,0] \oplus(4)[0,0,0,1,2] \oplus(3)[3,0,0,1,0] \oplus(8)[1,1,0,1,0] \oplus(4)[1,0,0,0,3] \oplus$ $[4,0,0,0,1] \oplus(5)[0,2,0,0,1] \oplus(11)[1,0,1,0,1] \oplus(7)[1,0,0,2,1] \oplus(7)[2,1,0,0,1] \oplus$ $(2)[0,1,0,3,0] \oplus[2,0,0,3,0] \oplus(8)[0,1,1,1,0] \oplus(3)[0,0,1,0,3] \oplus(5)[0,0,2,0,1] \oplus$ $(8)[0,1,0,1,2] \oplus(7)[2,0,1,1,0] \oplus[0,0,0,2,3] \oplus(4)[1,2,0,1,0] \oplus(2)[3,1,0,1,0] \oplus$ $(7)[2,0,0,1,2] \oplus(3)[0,0,1,2,1] \oplus[0,3,0,0,1] \oplus[3,0,0,0,3] \oplus(4)[1,1,0,0,3] \oplus$ $(3)[3,0,1,0,1] \oplus(9)[1,1,1,0,1] \oplus[2,2,0,0,1] \oplus[3,0,0,2,1] \oplus[1,0,0,1,4] \oplus$ $(3)[1,0,2,1,0] \oplus(4)[1,1,0,2,1] \oplus(2)[0,2,1,1,0] \oplus(4)[1,0,1,1,2] \oplus(2)[0,2,0,1,2] \oplus$ $(2)[0,1,2,0,1] \oplus[0,1,1,0,3] \oplus(2)[2,1,1,1,0] \oplus[2,0,2,0,1] \oplus(2)[2,1,0,1,2] \oplus$ $[2,0,1,0,3] \oplus[1,2,1,0,1]$

- Level-5: $[0,1,0,0,0] \oplus[2,0,0,0,0] \oplus(3)[0,0,0,1,1] \oplus[4,0,0,0,0] \oplus(4)[0,2,0,0,0] \oplus$ $(7)[1,0,1,0,0] \oplus(6)[1,0,0,2,0] \oplus(5)[1,0,0,0,2] \oplus(5)[2,1,0,0,0] \oplus[0,0,0,4,0] \oplus$ $[0,0,0,0,4] \oplus(6)[0,0,2,0,0] \oplus(13)[0,1,0,1,1] \oplus(6)[0,0,1,2,0] \oplus(7)[0,0,1,0,2] \oplus$ $(2)[0,3,0,0,0] \oplus(12)[2,0,0,1,1] \oplus(5)[0,0,0,2,2] \oplus[4,1,0,0,0] \oplus(6)[3,0,1,0,0] \oplus$ $(3)[2,2,0,0,0] \oplus(3)[3,0,0,2,0] \oplus(5)[3,0,0,0,2] \oplus(13)[1,1,1,0,0] \oplus(9)[1,1,0,2,0] \oplus$ $(12)[1,1,0,0,2] \oplus(3)[1,0,0,3,1] \oplus(7)[1,0,0,1,3] \oplus(2)[0,1,0,0,4] \oplus(6)[0,1,2,0,0] \oplus$ $(16)[1,0,1,1,1] \oplus(9)[0,2,0,1,1] \oplus(2)[4,0,0,1,1] \oplus(2)[2,0,0,0,4] \oplus(6)[2,0,2,0,0] \oplus$ $(4)[0,1,1,2,0] \oplus(8)[0,1,1,0,2] \oplus(11)[2,1,0,1,1] \oplus(4)[1,2,1,0,0] \oplus(4)[0,1,0,2,2] \oplus$ $(3)[2,0,1,2,0] \oplus(8)[2,0,1,0,2] \oplus(2)[3,1,1,0,0] \oplus(3)[0,0,2,1,1] \oplus(2)[0,0,1,1,3] \oplus$ $(2)[1,2,0,2,0] \oplus(4)[1,2,0,0,2] \oplus(3)[2,0,0,2,2] \oplus[3,1,0,2,0] \oplus(2)[3,1,0,0,2] \oplus$ $[1,0,3,0,0] \oplus[0,3,0,1,1] \oplus[0,2,2,0,0] \oplus[1,0,1,0,4] \oplus[3,0,0,1,3] \oplus(3)[1,1,0,1,3] \oplus$ $(2)[1,0,2,0,2] \oplus(2)[3,0,1,1,1] \oplus(6)[1,1,1,1,1] \oplus[0,2,1,0,2] \oplus[2,2,0,1,1] \oplus$ $[2,1,2,0,0] \oplus[2,1,1,0,2]$ 
- Level-6: $\quad[0,0,0,1,0] \oplus(3)[1,0,0,0,1] \oplus(7)[0,1,0,1,0] \oplus(2)[0,0,0,0,3] \oplus$ (6) $[2,0,0,1,0] \oplus(7)[0,0,1,0,1] \oplus(7)[0,0,0,2,1] \oplus(6)[3,0,0,0,1] \oplus(13)[1,1,0,0,1] \oplus$ $(5)[1,0,0,3,0] \oplus(3)[4,0,0,1,0] \oplus(10)[0,2,0,1,0] \oplus(17)[1,0,1,1,0] \oplus(14)[1,0,0,1,2] \oplus$ $(12)[2,1,0,1,0] \oplus(6)[0,1,0,0,3] \oplus[5,0,0,0,1] \oplus(7)[2,0,0,0,3] \oplus(16)[0,1,1,0,1] \oplus$ $(2)[0,0,0,1,4] \oplus(2)[0,0,1,3,0] \oplus(7)[0,0,2,1,0] \oplus(13)[0,1,0,2,1] \oplus(17)[2,0,1,0,1] \oplus$ $(2)[0,0,0,3,2] \oplus(11)[1,2,0,0,1] \oplus(7)[3,1,0,0,1] \oplus(11)[2,0,0,2,1] \oplus(9)[0,0,1,1,2] \oplus$ $[1,0,0,0,5] \oplus(3)[0,3,0,1,0] \oplus[3,0,0,3,0] \oplus(3)[1,1,0,3,0] \oplus(7)[3,0,1,1,0] \oplus$ $[4,1,0,1,0] \oplus(16)[1,1,1,1,0] \oplus(4)[2,2,0,1,0] \oplus(7)[3,0,0,1,2] \oplus(10)[1,0,2,0,1] \oplus$ (6) $[1,0,1,0,3] \oplus(3)[0,2,0,0,3] \oplus(16)[1,1,0,1,2] \oplus[4,0,0,0,3] \oplus(6)[0,2,1,0,1] \oplus$ $(3)[1,0,0,2,3] \oplus[1,3,0,0,1] \oplus(2)[4,0,1,0,1] \oplus(6)[1,0,1,2,1] \oplus(4)[0,2,0,2,1] \oplus$ (4) $[2,1,0,0,3] \oplus[0,0,3,0,1] \oplus[3,2,0,0,1] \oplus[4,0,0,2,1] \oplus(3)[0,1,2,1,0] \oplus[0,1,0,1,4] \oplus$ $[0,0,2,0,3] \oplus(9)[2,1,1,0,1] \oplus(3)[2,0,2,1,0] \oplus[2,0,0,1,4] \oplus(4)[2,1,0,2,1] \oplus$ $(4)[0,1,1,1,2] \oplus(2)[1,2,1,1,0] \oplus(4)[2,0,1,1,2] \oplus[3,1,1,1,0] \oplus(2)[1,2,0,1,2] \oplus$ $[3,1,0,1,2] \oplus[3,0,2,0,1] \oplus(2)[1,1,2,0,1] \oplus[1,1,1,0,3]$

- Level-7: $\quad[1,0,0,0,0] \oplus(4)[0,0,1,0,0] \oplus(3)[0,0,0,2,0] \oplus(2)[0,0,0,0,2] \oplus$ $(2)[3,0,0,0,0] \oplus(5)[1,1,0,0,0] \oplus(13)[1,0,0,1,1] \oplus[5,0,0,0,0] \oplus(12)[0,1,1,0,0] \oplus$ $(11)[0,1,0,2,0] \oplus(9)[0,1,0,0,2] \oplus(12)[2,0,1,0,0] \oplus(8)[1,2,0,0,0] \oplus(5)[3,1,0,0,0] \oplus$ $(9)[2,0,0,2,0] \oplus(9)[2,0,0,0,2] \oplus(5)[0,0,0,3,1] \oplus(5)[0,0,0,1,3] \oplus(15)[0,0,1,1,1] \oplus$ $[1,0,0,4,0] \oplus(3)[1,0,0,0,4] \oplus(13)[1,0,2,0,0] \oplus(12)[3,0,0,1,1] \oplus(10)[0,2,1,0,0] \oplus$ $(28)[1,1,0,1,1] \oplus(3)[1,3,0,0,0] \oplus(4)[4,0,1,0,0] \oplus(8)[0,2,0,2,0] \oplus(9)[0,2,0,0,2] \oplus$ $(13)[1,0,1,2,0] \oplus(15)[1,0,1,0,2] \oplus(2)[3,2,0,0,0] \oplus(2)[4,0,0,2,0] \oplus(4)[4,0,0,0,2] \oplus$ $(12)[1,0,0,2,2] \oplus(14)[2,1,1,0,0] \oplus(3)[0,0,3,0,0] \oplus(9)[2,1,0,2,0] \oplus(13)[2,1,0,0,2] \oplus$ $[0,0,1,0,4] \oplus[0,0,0,2,4] \oplus(2)[0,0,2,2,0] \oplus(4)[0,0,2,0,2] \oplus(4)[0,1,0,3,1] \oplus$ $(7)[0,1,0,1,3] \oplus(16)[0,1,1,1,1] \oplus[5,0,0,1,1] \oplus(3)[2,0,0,3,1] \oplus(7)[2,0,0,1,3] \oplus$ $[0,3,1,0,0] \oplus(3)[0,0,1,2,2] \oplus[3,0,0,0,4] \oplus(17)[2,0,1,1,1] \oplus(4)[3,0,2,0,0] \oplus$ $(2)[1,1,0,0,4] \oplus[0,3,0,2,0] \oplus[0,3,0,0,2] \oplus(7)[1,1,2,0,0] \oplus(11)[1,2,0,1,1] \oplus$ $(7)[3,1,0,1,1] \oplus[4,1,1,0,0] \oplus(2)[2,2,1,0,0] \oplus(2)[3,0,1,2,0] \oplus(5)[3,0,1,0,2] \oplus$ $(5)[1,1,1,2,0] \oplus(9)[1,1,1,0,2] \oplus[4,1,0,0,2] \oplus[2,2,0,2,0] \oplus(2)[2,2,0,0,2] \oplus$ $(2)[3,0,0,2,2] \oplus[0,1,3,0,0] \oplus(5)[1,1,0,2,2] \oplus(4)[1,0,2,1,1] \oplus(2)[1,0,1,1,3] \oplus$ $[0,2,0,1,3] \oplus[2,0,3,0,0] \oplus(2)[0,2,1,1,1] \oplus[0,1,2,0,2] \oplus[4,0,1,1,1] \oplus[2,1,0,1,3] \oplus$ $[2,0,2,0,2] \oplus(3)[2,1,1,1,1]$

- Level-8: $\quad(2)[0,0,0,0,1] \oplus(5)[1,0,0,1,0] \oplus(8)[0,1,0,0,1] \oplus(3)[0,0,0,3,0] \oplus$ $(6)[2,0,0,0,1] \oplus(10)[0,0,1,1,0] \oplus(7)[0,0,0,1,2] \oplus(6)[3,0,0,1,0] \oplus(16)[1,1,0,1,0] \oplus$ $(5)[1,0,0,0,3] \oplus(4)[4,0,0,0,1] \oplus(12)[0,2,0,0,1] \oplus(19)[1,0,1,0,1] \oplus(17)[1,0,0,2,1] \oplus$ $(15)[2,1,0,0,1] \oplus(6)[0,1,0,3,0] \oplus[5,0,0,1,0] \oplus(5)[2,0,0,3,0] \oplus(19)[0,1,1,1,0] \oplus$ $[0,0,0,4,1] \oplus(4)[0,0,1,0,3] \oplus(8)[0,0,2,0,1] \oplus(17)[0,1,0,1,2] \oplus(19)[2,0,1,1,0] \oplus$ (4) $[0,0,0,2,3] \oplus(14)[1,2,0,1,0] \oplus(8)[3,1,0,1,0] \oplus(16)[2,0,0,1,2] \oplus(10)[0,0,1,2,1] \oplus$ $(5)[0,3,0,0,1] \oplus(4)[3,0,0,0,3] \oplus(8)[1,1,0,0,3] \oplus(12)[3,0,1,0,1] \oplus(3)[4,1,0,0,1] \oplus$ $(22)[1,1,1,0,1] \oplus(7)[2,2,0,0,1] \oplus(7)[3,0,0,2,1] \oplus(2)[1,0,0,1,4] \oplus(10)[1,0,2,1,0] \oplus$ (3) $[1,0,1,3,0] \oplus(2)[0,2,0,3,0] \oplus(18)[1,1,0,2,1] \oplus(7)[0,2,1,1,0] \oplus(3)[1,0,0,3,2] \oplus$ $(2)[1,3,0,1,0] \oplus(3)[4,0,1,1,0] \oplus(12)[1,0,1,1,2] \oplus(7)[0,2,0,1,2] \oplus(2)[2,1,0,3,0] \oplus$ $[0,0,3,1,0] \oplus[3,2,0,1,0] \oplus(3)[4,0,0,1,2] \oplus(5)[0,1,2,0,1] \oplus(2)[0,1,1,0,3] \oplus$ 
$(10)[2,1,1,1,0] \oplus(2)[0,1,0,2,3] \oplus(6)[2,0,2,0,1] \oplus(10)[2,1,0,1,2] \oplus(3)[2,0,1,0,3] \oplus$ $[5,0,1,0,1] \oplus(4)[0,1,1,2,1] \oplus[0,0,2,1,2] \oplus[1,2,0,0,3] \oplus[2,0,0,2,3] \oplus[3,1,0,0,3] \oplus$ $(3)[1,2,1,0,1] \oplus(4)[2,0,1,2,1] \oplus(3)[3,1,1,0,1] \oplus(2)[1,2,0,2,1] \oplus[3,1,0,2,1] \oplus$ $[3,0,2,1,0] \oplus(2)[1,1,2,1,0] \oplus[1,0,3,0,1] \oplus[3,0,1,1,2] \oplus(2)[1,1,1,1,2]$

- Level-9: $[0,0,0,0,0] \oplus(3)[0,1,0,0,0] \oplus(2)[2,0,0,0,0] \oplus(6)[0,0,0,1,1] \oplus[4,0,0,0,0] \oplus$ (6) $[0,2,0,0,0] \oplus(10)[1,0,1,0,0] \oplus(8)[1,0,0,2,0] \oplus(7)[1,0,0,0,2] \oplus(6)[2,1,0,0,0] \oplus$ $[0,0,0,4,0] \oplus[0,0,0,0,4] \oplus(8)[0,0,2,0,0] \oplus(19)[0,1,0,1,1] \oplus(9)[0,0,1,2,0] \oplus$ $(8)[0,0,1,0,2] \oplus(5)[0,3,0,0,0] \oplus(16)[2,0,0,1,1] \oplus(8)[0,0,0,2,2] \oplus(2)[4,1,0,0,0] \oplus$ $(8)[3,0,1,0,0] \oplus(6)[2,2,0,0,0] \oplus(6)[3,0,0,2,0] \oplus(6)[3,0,0,0,2] \oplus(19)[1,1,1,0,0] \oplus$ $(16)[1,1,0,2,0] \oplus(15)[1,1,0,0,2] \oplus(8)[1,0,0,3,1] \oplus(8)[1,0,0,1,3] \oplus[0,4,0,0,0] \oplus$ $[0,1,0,4,0] \oplus[0,1,0,0,4] \oplus(8)[0,1,2,0,0] \oplus(24)[1,0,1,1,1] \oplus(16)[0,2,0,1,1] \oplus$ $(5)[4,0,0,1,1] \oplus[2,0,0,4,0] \oplus[2,0,0,0,4] \oplus[5,0,1,0,0] \oplus(9)[2,0,2,0,0] \oplus[2,3,0,0,0] \oplus$ $[5,0,0,0,2] \oplus(9)[0,1,1,2,0] \oplus(9)[0,1,1,0,2] \oplus(20)[2,1,0,1,1] \oplus[0,0,0,3,3] \oplus$ $(8)[1,2,1,0,0] \oplus(9)[0,1,0,2,2] \oplus(9)[2,0,1,2,0] \oplus(10)[2,0,1,0,2] \oplus(5)[3,1,1,0,0] \oplus$ (5) $[0,0,2,1,1] \oplus(2)[0,0,1,3,1] \oplus(2)[0,0,1,1,3] \oplus(6)[1,2,0,2,0] \oplus(7)[1,2,0,0,2] \oplus$ $(8)[2,0,0,2,2] \oplus(3)[3,1,0,2,0] \oplus(5)[3,1,0,0,2] \oplus(2)[1,0,3,0,0] \oplus(2)[0,3,0,1,1] \oplus$ $[0,2,2,0,0] \oplus[3,0,0,3,1] \oplus(2)[3,0,0,1,3] \oplus(3)[1,1,0,3,1] \oplus(4)[1,1,0,1,3] \oplus$ $(2)[1,0,2,2,0] \oplus(2)[1,0,2,0,2] \oplus[4,0,2,0,0] \oplus(6)[3,0,1,1,1] \oplus(12)[1,1,1,1,1] \oplus$ $[4,1,0,1,1] \oplus[0,2,1,2,0] \oplus[0,2,1,0,2] \oplus(3)[2,2,0,1,1] \oplus(2)[2,1,2,0,0] \oplus[4,0,1,0,2] \oplus$ $(2)[1,0,1,2,2] \oplus[0,2,0,2,2] \oplus[2,1,1,2,0] \oplus(2)[2,1,1,0,2] \oplus[0,1,2,1,1] \oplus[2,1,0,2,2] \oplus$ $[2,0,2,1,1]$

- Level-10: $\quad(2)[0,0,0,1,0] \oplus(5)[1,0,0,0,1] \oplus(8)[0,1,0,1,0] \oplus(3)[0,0,0,0,3] \oplus$ (6) $[2,0,0,1,0] \oplus(9)[0,0,1,0,1] \oplus(7)[0,0,0,2,1] \oplus(4)[3,0,0,0,1] \oplus(14)[1,1,0,0,1] \oplus$ $(5)[1,0,0,3,0] \oplus(2)[4,0,0,1,0] \oplus(11)[0,2,0,1,0] \oplus(17)[1,0,1,1,0] \oplus(14)[1,0,0,1,2] \oplus$ $(12)[2,1,0,1,0] \oplus(5)[0,1,0,0,3] \oplus[5,0,0,0,1] \oplus(4)[2,0,0,0,3] \oplus(16)[0,1,1,0,1] \oplus$ $[0,0,0,1,4] \oplus(3)[0,0,1,3,0] \oplus(7)[0,0,2,1,0] \oplus(14)[0,1,0,2,1] \oplus(14)[2,0,1,0,1] \oplus$ $(3)[0,0,0,3,2] \oplus(12)[1,2,0,0,1] \oplus(6)[3,1,0,0,1] \oplus(13)[2,0,0,2,1] \oplus(8)[0,0,1,1,2] \oplus$ $(4)[0,3,0,1,0] \oplus(2)[3,0,0,3,0] \oplus(5)[1,1,0,3,0] \oplus(7)[3,0,1,1,0] \oplus[4,1,0,1,0] \oplus$ $(17)[1,1,1,1,0] \oplus(5)[2,2,0,1,0] \oplus(6)[3,0,0,1,2] \oplus[1,0,0,4,1] \oplus(7)[1,0,2,0,1] \oplus$ $(2)[1,0,1,0,3] \oplus(2)[0,2,0,0,3] \oplus(15)[1,1,0,1,2] \oplus[4,0,0,0,3] \oplus(6)[0,2,1,0,1] \oplus$ $(3)[1,0,0,2,3] \oplus(2)[1,3,0,0,1] \oplus(2)[4,0,1,0,1] \oplus(9)[1,0,1,2,1] \oplus(5)[0,2,0,2,1] \oplus$ $(2)[2,1,0,0,3] \oplus[3,2,0,0,1] \oplus[4,0,0,2,1] \oplus(3)[0,1,2,1,0] \oplus[0,1,1,3,0] \oplus$ $(8)[2,1,1,0,1] \oplus[0,1,0,3,2] \oplus(3)[2,0,2,1,0] \oplus(6)[2,1,0,2,1] \oplus[2,0,1,3,0] \oplus$ $(3)[0,1,1,1,2] \oplus[0,0,2,2,1] \oplus[2,0,0,3,2] \oplus(2)[1,2,1,1,0] \oplus(3)[2,0,1,1,2] \oplus$ $[3,1,1,1,0] \oplus(2)[1,2,0,1,2] \oplus[3,1,0,1,2] \oplus[3,0,2,0,1] \oplus[1,1,2,0,1] \oplus[1,1,1,2,1]$

- Level-11: $[1,0,0,0,0] \oplus(4)[0,0,1,0,0] \oplus(2)[0,0,0,2,0] \oplus(3)[0,0,0,0,2] \oplus[3,0,0,0,0] \oplus$ $(5)[1,1,0,0,0] \oplus(11)[1,0,0,1,1] \oplus(10)[0,1,1,0,0] \oplus(7)[0,1,0,2,0] \oplus(9)[0,1,0,0,2] \oplus$ $(8)[2,0,1,0,0] \oplus(6)[1,2,0,0,0] \oplus(2)[3,1,0,0,0] \oplus(6)[2,0,0,2,0] \oplus(6)[2,0,0,0,2] \oplus$ (3) $[0,0,0,3,1] \oplus(4)[0,0,0,1,3] \oplus(11)[0,0,1,1,1] \oplus[1,0,0,4,0] \oplus[1,0,0,0,4] \oplus$ $(8)[1,0,2,0,0] \oplus(6)[3,0,0,1,1] \oplus(7)[0,2,1,0,0] \oplus(19)[1,1,0,1,1] \oplus(2)[1,3,0,0,0] \oplus$ $[4,0,1,0,0] \oplus(5)[0,2,0,2,0] \oplus(6)[0,2,0,0,2] \oplus(9)[1,0,1,2,0] \oplus(9)[1,0,1,0,2] \oplus$ 
$[3,2,0,0,0] \oplus[4,0,0,2,0] \oplus[4,0,0,0,2] \oplus(8)[1,0,0,2,2] \oplus(8)[2,1,1,0,0] \oplus[0,0,3,0,0] \oplus$ (6) $[2,1,0,2,0] \oplus(6)[2,1,0,0,2] \oplus(2)[0,0,2,2,0] \oplus[0,0,2,0,2] \oplus(3)[0,1,0,3,1] \oplus$ $(3)[0,1,0,1,3] \oplus(10)[0,1,1,1,1] \oplus(3)[2,0,0,3,1] \oplus(2)[2,0,0,1,3] \oplus[0,3,1,0,0] \oplus$ $(2)[0,0,1,2,2] \oplus(9)[2,0,1,1,1] \oplus[3,0,2,0,0] \oplus[0,3,0,0,2] \oplus(3)[1,1,2,0,0] \oplus$ $(7)[1,2,0,1,1] \oplus(3)[3,1,0,1,1] \oplus[2,2,1,0,0] \oplus[3,0,1,2,0] \oplus[3,0,1,0,2] \oplus$ $(3)[1,1,1,2,0] \oplus(3)[1,1,1,0,2] \oplus[2,2,0,0,2] \oplus[3,0,0,2,2] \oplus(3)[1,1,0,2,2] \oplus$ $[1,0,2,1,1] \oplus[1,0,1,3,1] \oplus[0,2,1,1,1] \oplus[2,1,1,1,1]$

- Level-12: $[0,0,0,0,1] \oplus(3)[1,0,0,1,0] \oplus(6)[0,1,0,0,1] \oplus[0,0,0,3,0] \oplus(4)[2,0,0,0,1] \oplus$ (5) $[0,0,1,1,0] \oplus(5)[0,0,0,1,2] \oplus(2)[3,0,0,1,0] \oplus(8)[1,1,0,1,0] \oplus(4)[1,0,0,0,3] \oplus$ $(6)[0,2,0,0,1] \oplus(11)[1,0,1,0,1] \oplus(7)[1,0,0,2,1] \oplus(6)[2,1,0,0,1] \oplus(2)[0,1,0,3,0] \oplus$ $(2)[2,0,0,3,0] \oplus(8)[0,1,1,1,0] \oplus(2)[0,0,1,0,3] \oplus(4)[0,0,2,0,1] \oplus(8)[0,1,0,1,2] \oplus$ $(7)[2,0,1,1,0] \oplus[0,0,0,2,3] \oplus(5)[1,2,0,1,0] \oplus(2)[3,1,0,1,0] \oplus(6)[2,0,0,1,2] \oplus$ $(4)[0,0,1,2,1] \oplus(2)[0,3,0,0,1] \oplus(2)[1,1,0,0,3] \oplus(2)[3,0,1,0,1] \oplus(8)[1,1,1,0,1] \oplus$ $(2)[2,2,0,0,1] \oplus(2)[3,0,0,2,1] \oplus(3)[1,0,2,1,0] \oplus[1,0,1,3,0] \oplus(6)[1,1,0,2,1] \oplus$ $(2)[0,2,1,1,0] \oplus[1,0,0,3,2] \oplus(3)[1,0,1,1,2] \oplus(2)[0,2,0,1,2] \oplus[0,1,2,0,1] \oplus$ $(2)[2,1,1,1,0] \oplus(2)[2,1,0,1,2] \oplus[0,1,1,2,1] \oplus[1,2,1,0,1] \oplus[2,0,1,2,1]$

- Level-13: $[0,1,0,0,0] \oplus[2,0,0,0,0] \oplus(2)[0,0,0,1,1] \oplus(2)[0,2,0,0,0] \oplus(4)[1,0,1,0,0] \oplus$ $(2)[1,0,0,2,0] \oplus(4)[1,0,0,0,2] \oplus(2)[2,1,0,0,0] \oplus[0,0,0,0,4] \oplus(3)[0,0,2,0,0] \oplus$ (6) $[0,1,0,1,1] \oplus(2)[0,0,1,2,0] \oplus(4)[0,0,1,0,2] \oplus[0,3,0,0,0] \oplus(5)[2,0,0,1,1] \oplus$ $(2)[0,0,0,2,2] \oplus[3,0,1,0,0] \oplus[2,2,0,0,0] \oplus[3,0,0,2,0] \oplus[3,0,0,0,2] \oplus(5)[1,1,1,0,0] \oplus$ $(3)[1,1,0,2,0] \oplus(5)[1,1,0,0,2] \oplus[1,0,0,3,1] \oplus(2)[1,0,0,1,3] \oplus(2)[0,1,2,0,0] \oplus$ (6) $[1,0,1,1,1] \oplus(3)[0,2,0,1,1] \oplus[2,0,2,0,0] \oplus[0,1,1,2,0] \oplus(2)[0,1,1,0,2] \oplus$ (3) $[2,1,0,1,1] \oplus[1,2,1,0,0] \oplus[0,1,0,2,2] \oplus[2,0,1,2,0] \oplus[2,0,1,0,2] \oplus[0,0,2,1,1] \oplus$ $[1,2,0,0,2] \oplus[2,0,0,2,2] \oplus[1,1,1,1,1]$

- Level-14: $[1,0,0,0,1] \oplus[0,1,0,1,0] \oplus[0,0,0,0,3] \oplus[2,0,0,1,0] \oplus(2)[0,0,1,0,1] \oplus$ $[0,0,0,2,1] \oplus[3,0,0,0,1] \oplus(3)[1,1,0,0,1] \oplus[0,2,0,1,0] \oplus(3)[1,0,1,1,0] \oplus$ $(3)[1,0,0,1,2] \oplus[2,1,0,1,0] \oplus[0,1,0,0,3] \oplus[2,0,0,0,3] \oplus(3)[0,1,1,0,1] \oplus[0,0,2,1,0] \oplus$ $[0,1,0,2,1] \oplus(2)[2,0,1,0,1] \oplus[1,2,0,0,1] \oplus[2,0,0,2,1] \oplus[0,0,1,1,2] \oplus[1,1,1,1,0] \oplus$ $[1,0,2,0,1] \oplus[1,1,0,1,2]$

- Level-15: $\quad[1,0,0,1,1] \oplus[0,1,1,0,0] \oplus[0,1,0,0,2] \oplus[2,0,1,0,0] \oplus[2,0,0,0,2] \oplus$ $[0,0,1,1,1] \oplus[1,0,2,0,0] \oplus[1,1,0,1,1] \oplus[1,0,1,0,2]$

- Level-16: $[1,0,1,0,1]$

\section{B.3 Dynkin Label library of $\mathcal{V}_{[3,0,0,0,1]}$}

- Level-0: $[3,0,0,0,1]$

- Level-1: $[3,0,0,0,0] \oplus[2,0,1,0,0] \oplus[3,1,0,0,0] \oplus[2,0,0,0,2] \oplus[3,0,0,1,1]$

- Level-2: $\quad[2,0,0,0,1] \oplus(2)[3,0,0,1,0] \oplus[1,1,0,1,0] \oplus[4,0,0,0,1] \oplus[1,0,1,0,1] \oplus$ $(2)[2,1,0,0,1] \oplus[2,0,1,1,0] \oplus[3,1,0,1,0] \oplus[2,0,0,1,2] \oplus[3,0,1,0,1]$ 
- Level-3: $\quad[4,0,0,0,0] \oplus[0,2,0,0,0] \oplus[1,0,1,0,0] \oplus[1,0,0,2,0] \oplus(2)[2,1,0,0,0] \oplus$ $[0,1,0,1,1] \oplus(3)[2,0,0,1,1] \oplus[4,1,0,0,0] \oplus(3)[3,0,1,0,0] \oplus[2,2,0,0,0] \oplus[3,0,0,2,0] \oplus$ $(2)[3,0,0,0,2] \oplus(2)[1,1,1,0,0] \oplus[1,1,0,2,0] \oplus[1,1,0,0,2] \oplus[1,0,1,1,1] \oplus[4,0,0,1,1] \oplus$ $[2,0,2,0,0] \oplus(2)[2,1,0,1,1] \oplus[2,0,1,0,2] \oplus[3,1,1,0,0] \oplus[3,1,0,0,2]$

- Level-4: $\quad(2)[0,1,0,1,0] \oplus(2)[2,0,0,1,0] \oplus[0,0,0,2,1] \oplus(3)[3,0,0,0,1] \oplus$ $(3)[1,1,0,0,1] \oplus[1,0,0,3,0] \oplus(2)[4,0,0,1,0] \oplus(2)[0,2,0,1,0] \oplus(3)[1,0,1,1,0] \oplus$ $[1,0,0,1,2] \oplus(4)[2,1,0,1,0] \oplus[5,0,0,0,1] \oplus[2,0,0,0,3] \oplus[0,1,1,0,1] \oplus[0,1,0,2,1] \oplus$ $(4)[2,0,1,0,1] \oplus(2)[1,2,0,0,1] \oplus(4)[3,1,0,0,1] \oplus(2)[2,0,0,2,1] \oplus(2)[3,0,1,1,0] \oplus$ $[4,1,0,1,0] \oplus(2)[1,1,1,1,0] \oplus[2,2,0,1,0] \oplus(2)[3,0,0,1,2] \oplus[1,0,2,0,1] \oplus[1,1,0,1,2] \oplus$ $[4,0,0,0,3] \oplus[4,0,1,0,1] \oplus[2,1,0,0,3] \oplus[3,2,0,0,1] \oplus(2)[2,1,1,0,1]$

- Level-5: $[0,0,1,0,0] \oplus[0,0,0,2,0] \oplus[3,0,0,0,0] \oplus(2)[1,1,0,0,0] \oplus(3)[1,0,0,1,1] \oplus$ $[5,0,0,0,0] \oplus(3)[0,1,1,0,0] \oplus(3)[0,1,0,2,0] \oplus[0,1,0,0,2] \oplus(4)[2,0,1,0,0] \oplus$ $(3)[1,2,0,0,0] \oplus(3)[3,1,0,0,0] \oplus(3)[2,0,0,2,0] \oplus(2)[2,0,0,0,2] \oplus[0,0,0,3,1] \oplus$ $[0,0,1,1,1] \oplus(2)[1,0,2,0,0] \oplus(5)[3,0,0,1,1] \oplus[5,1,0,0,0] \oplus(2)[0,2,1,0,0] \oplus$ $(6)[1,1,0,1,1] \oplus[1,3,0,0,0] \oplus(3)[4,0,1,0,0] \oplus[0,2,0,2,0] \oplus[0,2,0,0,2] \oplus$ $(2)[1,0,1,2,0] \oplus[1,0,1,0,2] \oplus(2)[3,2,0,0,0] \oplus[4,0,0,2,0] \oplus(3)[4,0,0,0,2] \oplus$ $[1,0,0,2,2] \oplus(5)[2,1,1,0,0] \oplus(2)[2,1,0,2,0] \oplus(4)[2,1,0,0,2] \oplus[0,1,1,1,1] \oplus$ $[5,0,0,1,1] \oplus[2,0,0,1,3] \oplus[3,0,0,0,4] \oplus(3)[2,0,1,1,1] \oplus[3,0,2,0,0] \oplus[1,1,2,0,0] \oplus$ $(2)[1,2,0,1,1] \oplus(3)[3,1,0,1,1] \oplus[4,1,1,0,0] \oplus[2,2,1,0,0] \oplus(2)[3,0,1,0,2] \oplus$ $[1,1,1,0,2] \oplus[4,1,0,0,2] \oplus[2,2,0,0,2]$

- Level-6: $[0,0,0,0,1] \oplus(2)[1,0,0,1,0] \oplus(3)[0,1,0,0,1] \oplus[0,0,0,3,0] \oplus(2)[2,0,0,0,1] \oplus$ (3) $[0,0,1,1,0] \oplus[0,0,0,1,2] \oplus(3)[3,0,0,1,0] \oplus(6)[1,1,0,1,0] \oplus(3)[4,0,0,0,1] \oplus$ $(4)[0,2,0,0,1] \oplus(4)[1,0,1,0,1] \oplus(4)[1,0,0,2,1] \oplus(6)[2,1,0,0,1] \oplus[0,1,0,3,0] \oplus$ $(2)[5,0,0,1,0] \oplus[2,0,0,3,0] \oplus(4)[0,1,1,1,0] \oplus(2)[0,1,0,1,2] \oplus(6)[2,0,1,1,0] \oplus$ $(5)[1,2,0,1,0] \oplus(5)[3,1,0,1,0] \oplus[6,0,0,0,1] \oplus(4)[2,0,0,1,2] \oplus[0,0,1,2,1] \oplus$ $(2)[0,3,0,0,1] \oplus(2)[3,0,0,0,3] \oplus[1,1,0,0,3] \oplus(6)[3,0,1,0,1] \oplus(4)[4,1,0,0,1] \oplus$ $(5)[1,1,1,0,1] \oplus(4)[2,2,0,0,1] \oplus(2)[3,0,0,2,1] \oplus[1,0,2,1,0] \oplus(3)[1,1,0,2,1] \oplus$ $[0,2,1,1,0] \oplus[5,1,0,1,0] \oplus[1,3,0,1,0] \oplus(2)[4,0,1,1,0] \oplus[1,0,1,1,2] \oplus[0,2,0,1,2] \oplus$ $[3,2,0,1,0] \oplus(2)[4,0,0,1,2] \oplus(3)[2,1,1,1,0] \oplus[2,0,2,0,1] \oplus(3)[2,1,0,1,2] \oplus$ $[2,0,1,0,3] \oplus[5,0,1,0,1] \oplus[3,1,0,0,3] \oplus[1,2,1,0,1] \oplus(2)[3,1,1,0,1]$

- Level-7: $[0,0,0,0,0] \oplus(2)[0,1,0,0,0] \oplus[2,0,0,0,0] \oplus(3)[0,0,0,1,1] \oplus[4,0,0,0,0] \oplus$ $(3)[0,2,0,0,0] \oplus(4)[1,0,1,0,0] \oplus(3)[1,0,0,2,0] \oplus(2)[1,0,0,0,2] \oplus(3)[2,1,0,0,0] \oplus$ $(2)[0,0,2,0,0] \oplus[6,0,0,0,0] \oplus(6)[0,1,0,1,1] \oplus(2)[0,0,1,2,0] \oplus[0,0,1,0,2] \oplus$ $(3)[0,3,0,0,0] \oplus(6)[2,0,0,1,1] \oplus[0,0,0,2,2] \oplus(3)[4,1,0,0,0] \oplus(5)[3,0,1,0,0] \oplus$ $(4)[2,2,0,0,0] \oplus(3)[3,0,0,2,0] \oplus(3)[3,0,0,0,2] \oplus(7)[1,1,1,0,0] \oplus(5)[1,1,0,2,0] \oplus$ (4) $[1,1,0,0,2] \oplus[1,0,0,3,1] \oplus[1,0,0,1,3] \oplus[0,4,0,0,0] \oplus[0,1,2,0,0] \oplus[6,1,0,0,0] \oplus$ $(5)[1,0,1,1,1] \oplus(5)[0,2,0,1,1] \oplus(5)[4,0,0,1,1] \oplus(3)[5,0,1,0,0] \oplus(3)[2,0,2,0,0] \oplus$ $[4,2,0,0,0] \oplus[2,3,0,0,0] \oplus[5,0,0,2,0] \oplus(2)[5,0,0,0,2] \oplus[0,1,1,2,0] \oplus[0,1,1,0,2] \oplus$ $(9)[2,1,0,1,1] \oplus(4)[1,2,1,0,0] \oplus[0,1,0,2,2] \oplus(2)[2,0,1,2,0] \oplus(3)[2,0,1,0,2] \oplus$ $(5)[3,1,1,0,0] \oplus(2)[1,2,0,2,0] \oplus(3)[1,2,0,0,2] \oplus(2)[2,0,0,2,2] \oplus(2)[3,1,0,2,0] \oplus$ 
(4) $[3,1,0,0,2] \oplus[6,0,0,1,1] \oplus[0,3,0,1,1] \oplus[3,0,0,1,3] \oplus[1,1,0,1,3] \oplus[4,0,2,0,0] \oplus$ $(3)[3,0,1,1,1] \oplus(2)[1,1,1,1,1] \oplus(2)[4,1,0,1,1] \oplus(2)[2,2,0,1,1] \oplus[2,1,2,0,0] \oplus$ $[4,0,1,0,2] \oplus[2,1,1,0,2]$

- Level-8: $\quad(2)[0,0,0,1,0] \oplus(3)[1,0,0,0,1] \oplus(4)[0,1,0,1,0] \oplus[0,0,0,0,3] \oplus$ $(3)[2,0,0,1,0] \oplus(4)[0,0,1,0,1] \oplus(2)[0,0,0,2,1] \oplus(3)[3,0,0,0,1] \oplus(6)[1,1,0,0,1] \oplus$ $[1,0,0,3,0] \oplus(3)[4,0,0,1,0] \oplus(5)[0,2,0,1,0] \oplus(6)[1,0,1,1,0] \oplus(4)[1,0,0,1,2] \oplus$ $(7)[2,1,0,1,0] \oplus[0,1,0,0,3] \oplus(3)[5,0,0,0,1] \oplus[2,0,0,0,3] \oplus(5)[0,1,1,0,1] \oplus$ $[0,0,2,1,0] \oplus(3)[0,1,0,2,1] \oplus(6)[2,0,1,0,1] \oplus(7)[1,2,0,0,1] \oplus(6)[3,1,0,0,1] \oplus$ $(2)[6,0,0,1,0] \oplus(5)[2,0,0,2,1] \oplus[0,0,1,1,2] \oplus(3)[0,3,0,1,0] \oplus[3,0,0,3,0] \oplus$ $[1,1,0,3,0] \oplus[7,0,0,0,1] \oplus(6)[3,0,1,1,0] \oplus(4)[4,1,0,1,0] \oplus(6)[1,1,1,1,0] \oplus$ $(5)[2,2,0,1,0] \oplus(4)[3,0,0,1,2] \oplus[1,0,2,0,1] \oplus[0,2,0,0,3] \oplus(5)[1,1,0,1,2] \oplus$ $[4,0,0,0,3] \oplus(2)[0,2,1,0,1] \oplus[1,0,0,2,3] \oplus(2)[5,1,0,0,1] \oplus(2)[1,3,0,0,1] \oplus$ $(4)[4,0,1,0,1] \oplus[1,0,1,2,1] \oplus[0,2,0,2,1] \oplus[2,1,0,0,3] \oplus(2)[3,2,0,0,1] \oplus$ $(2)[4,0,0,2,1] \oplus(5)[2,1,1,0,1] \oplus[2,0,2,1,0] \oplus(3)[2,1,0,2,1] \oplus[5,0,1,1,0] \oplus$ $[5,0,0,1,2] \oplus[1,2,1,1,0] \oplus[2,0,1,1,2] \oplus(2)[3,1,1,1,0] \oplus[1,2,0,1,2] \oplus[3,1,0,1,2] \oplus$ $[3,0,2,0,1]$

- Level-9: $[1,0,0,0,0] \oplus(3)[0,0,1,0,0] \oplus[0,0,0,2,0] \oplus(2)[0,0,0,0,2] \oplus[3,0,0,0,0] \oplus$ (3) $[1,1,0,0,0] \oplus(5)[1,0,0,1,1] \oplus[5,0,0,0,0] \oplus(5)[0,1,1,0,0] \oplus(2)[0,1,0,2,0] \oplus$ (4) $[0,1,0,0,2] \oplus(5)[2,0,1,0,0] \oplus(4)[1,2,0,0,0] \oplus(3)[3,1,0,0,0] \oplus(3)[2,0,0,2,0] \oplus$ (3) $[2,0,0,0,2] \oplus[0,0,0,1,3] \oplus[7,0,0,0,0] \oplus(3)[0,0,1,1,1] \oplus(3)[1,0,2,0,0] \oplus$ (6) $[3,0,0,1,1] \oplus(2)[5,1,0,0,0] \oplus(4)[0,2,1,0,0] \oplus(9)[1,1,0,1,1] \oplus(3)[1,3,0,0,0] \oplus$ $(4)[4,0,1,0,0] \oplus(2)[0,2,0,2,0] \oplus(3)[0,2,0,0,2] \oplus(2)[1,0,1,2,0] \oplus(3)[1,0,1,0,2] \oplus$ $(3)[3,2,0,0,0] \oplus(3)[4,0,0,2,0] \oplus(2)[4,0,0,0,2] \oplus(2)[1,0,0,2,2] \oplus(7)[2,1,1,0,0] \oplus$ $(5)[2,1,0,2,0] \oplus(4)[2,1,0,0,2] \oplus[0,1,0,1,3] \oplus(2)[0,1,1,1,1] \oplus(3)[5,0,0,1,1] \oplus$ $[2,0,0,3,1] \oplus[2,0,0,1,3] \oplus[6,0,1,0,0] \oplus[0,3,1,0,0] \oplus[6,0,0,0,2] \oplus(5)[2,0,1,1,1] \oplus$ $(2)[3,0,2,0,0] \oplus[0,3,0,0,2] \oplus[1,1,2,0,0] \oplus(5)[1,2,0,1,1] \oplus(6)[3,1,0,1,1] \oplus$ $(2)[4,1,1,0,0] \oplus(2)[2,2,1,0,0] \oplus(2)[3,0,1,2,0] \oplus[3,0,1,0,2] \oplus[1,1,1,2,0] \oplus$ $[1,1,1,0,2] \oplus[4,1,0,2,0] \oplus[4,1,0,0,2] \oplus[2,2,0,2,0] \oplus[2,2,0,0,2] \oplus[3,0,0,2,2] \oplus$ $[1,1,0,2,2] \oplus[4,0,1,1,1] \oplus[2,1,1,1,1]$

- Level-10: $\quad[0,0,0,0,1] \oplus(2)[1,0,0,1,0] \oplus(4)[0,1,0,0,1] \oplus(3)[2,0,0,0,1] \oplus$ $(2)[0,0,1,1,0] \oplus(2)[0,0,0,1,2] \oplus(3)[3,0,0,1,0] \oplus(5)[1,1,0,1,0] \oplus(2)[1,0,0,0,3] \oplus$ $(2)[4,0,0,0,1] \oplus(4)[0,2,0,0,1] \oplus(6)[1,0,1,0,1] \oplus(2)[1,0,0,2,1] \oplus(6)[2,1,0,0,1] \oplus$ $(2)[5,0,0,1,0] \oplus[2,0,0,3,0] \oplus(3)[0,1,1,1,0] \oplus[0,0,1,0,3] \oplus[0,0,2,0,1] \oplus$ $(3)[0,1,0,1,2] \oplus(6)[2,0,1,1,0] \oplus(5)[1,2,0,1,0] \oplus(6)[3,1,0,1,0] \oplus[6,0,0,0,1] \oplus$ $(4)[2,0,0,1,2] \oplus(2)[0,3,0,0,1] \oplus[1,1,0,0,3] \oplus(4)[3,0,1,0,1] \oplus(3)[4,1,0,0,1] \oplus$ $(5)[1,1,1,0,1] \oplus(4)[2,2,0,0,1] \oplus(4)[3,0,0,2,1] \oplus[1,0,2,1,0] \oplus(3)[1,1,0,2,1] \oplus$ $[4,0,0,3,0] \oplus[0,2,1,1,0] \oplus[5,1,0,1,0] \oplus[1,3,0,1,0] \oplus(3)[4,0,1,1,0] \oplus[1,0,1,1,2] \oplus$ $[0,2,0,1,2] \oplus[2,1,0,3,0] \oplus(2)[3,2,0,1,0] \oplus[4,0,0,1,2] \oplus(4)[2,1,1,1,0] \oplus$ $(2)[2,1,0,1,2] \oplus[5,0,1,0,1] \oplus[1,2,1,0,1] \oplus[2,0,1,2,1] \oplus[3,1,1,0,1] \oplus[3,1,0,2,1]$

- Level-11: $[0,1,0,0,0] \oplus[2,0,0,0,0] \oplus[0,0,0,1,1] \oplus[4,0,0,0,0] \oplus(2)[0,2,0,0,0] \oplus$ $(3)[1,0,1,0,0] \oplus[1,0,0,2,0] \oplus(3)[1,0,0,0,2] \oplus(3)[2,1,0,0,0] \oplus[0,0,0,0,4] \oplus$ 
$[0,0,2,0,0] \oplus(3)[0,1,0,1,1] \oplus(2)[0,0,1,0,2] \oplus[0,3,0,0,0] \oplus(5)[2,0,0,1,1] \oplus$ $(2)[4,1,0,0,0] \oplus(4)[3,0,1,0,0] \oplus(3)[2,2,0,0,0] \oplus(3)[3,0,0,2,0] \oplus(2)[3,0,0,0,2] \oplus$ $(5)[1,1,1,0,0] \oplus(2)[1,1,0,2,0] \oplus(4)[1,1,0,0,2] \oplus[1,0,0,1,3] \oplus[0,1,2,0,0] \oplus$ $(3)[1,0,1,1,1] \oplus(2)[0,2,0,1,1] \oplus(3)[4,0,0,1,1] \oplus[5,0,1,0,0] \oplus(2)[2,0,2,0,0] \oplus$ $[4,2,0,0,0] \oplus[2,3,0,0,0] \oplus[5,0,0,2,0] \oplus[0,1,1,0,2] \oplus(6)[2,1,0,1,1] \oplus(2)[1,2,1,0,0] \oplus$

$(2)[2,0,1,2,0] \oplus[2,0,1,0,2] \oplus(3)[3,1,1,0,0] \oplus[1,2,0,2,0] \oplus[1,2,0,0,2] \oplus[2,0,0,2,2] \oplus$ $(3)[3,1,0,2,0] \oplus[3,1,0,0,2] \oplus[3,0,0,3,1] \oplus[3,0,1,1,1] \oplus[1,1,1,1,1] \oplus[4,1,0,1,1] \oplus$ $[2,2,0,1,1]$

- Level-12: $\quad[1,0,0,0,1] \oplus[0,1,0,1,0] \oplus[0,0,0,0,3] \oplus(2)[2,0,0,1,0] \oplus[0,0,1,0,1] \oplus$ $(3)[3,0,0,0,1] \oplus(4)[1,1,0,0,1] \oplus(2)[4,0,0,1,0] \oplus[0,2,0,1,0] \oplus(2)[1,0,1,1,0] \oplus$ $(2)[1,0,0,1,2] \oplus(4)[2,1,0,1,0] \oplus[0,1,0,0,3] \oplus[2,0,0,0,3] \oplus(2)[0,1,1,0,1] \oplus$ (4) $[2,0,1,0,1] \oplus(2)[1,2,0,0,1] \oplus(3)[3,1,0,0,1] \oplus(2)[2,0,0,2,1] \oplus[3,0,0,3,0] \oplus$ $(3)[3,0,1,1,0] \oplus(2)[4,1,0,1,0] \oplus(2)[1,1,1,1,0] \oplus(2)[2,2,0,1,0] \oplus[3,0,0,1,2] \oplus$ $[1,0,2,0,1] \oplus[1,1,0,1,2] \oplus[3,2,0,0,1] \oplus[4,0,0,2,1] \oplus[2,1,1,0,1] \oplus[2,1,0,2,1]$

- Level-13: $\quad[3,0,0,0,0] \oplus[1,1,0,0,0] \oplus[1,0,0,1,1] \oplus[0,1,1,0,0] \oplus[0,1,0,0,2] \oplus$ $(3)[2,0,1,0,0] \oplus[1,2,0,0,0] \oplus(2)[3,1,0,0,0] \oplus[2,0,0,2,0] \oplus(2)[2,0,0,0,2] \oplus$ $[1,0,2,0,0] \oplus(3)[3,0,0,1,1] \oplus(2)[1,1,0,1,1] \oplus[4,0,1,0,0] \oplus[1,0,1,0,2] \oplus[3,2,0,0,0] \oplus$ $[4,0,0,2,0] \oplus(2)[2,1,1,0,0] \oplus[2,1,0,2,0] \oplus[2,1,0,0,2] \oplus[2,0,1,1,1] \oplus[3,1,0,1,1]$

- Level-14: $[2,0,0,0,1] \oplus(2)[3,0,0,1,0] \oplus[1,1,0,1,0] \oplus[4,0,0,0,1] \oplus[1,0,1,0,1] \oplus$ $(2)[2,1,0,0,1] \oplus[2,0,1,1,0] \oplus[3,1,0,1,0] \oplus[2,0,0,1,2] \oplus[3,0,1,0,1]$

- Level-15: $[4,0,0,0,0] \oplus[2,1,0,0,0] \oplus[2,0,0,1,1] \oplus[3,0,1,0,0] \oplus[3,0,0,0,2]$

- Level-16: [3, 0, 0, 0, 1]

\section{B.4 Dynkin Label library of $\mathcal{V}_{[4,0,0,0,0]}$}

- Level-0: $[4,0,0,0,0]$

- Level-1: $[3,0,0,0,1] \oplus[4,0,0,1,0]$

- Level-2: $[2,0,1,0,0] \oplus[3,1,0,0,0] \oplus[3,0,0,1,1] \oplus[4,0,1,0,0]$

- Level-3: $\quad[3,0,0,1,0] \oplus[1,1,0,1,0] \oplus[4,0,0,0,1] \oplus[2,1,0,0,1] \oplus[2,0,1,1,0] \oplus$ $[3,1,0,1,0] \oplus[3,0,1,0,1] \oplus[4,1,0,0,1]$

- Level-4: $\quad[4,0,0,0,0] \oplus[0,2,0,0,0] \oplus[1,0,0,2,0] \oplus[2,1,0,0,0] \oplus[2,0,0,1,1] \oplus$ $[4,1,0,0,0] \oplus[3,0,1,0,0] \oplus[2,2,0,0,0] \oplus[3,0,0,2,0] \oplus[3,0,0,0,2] \oplus[1,1,1,0,0] \oplus$ $[1,1,0,2,0] \oplus[4,0,0,1,1] \oplus[2,0,2,0,0] \oplus[4,2,0,0,0] \oplus[5,0,0,0,2] \oplus[2,1,0,1,1] \oplus$ $[3,1,1,0,0] \oplus[3,1,0,0,2]$

- Level-5: $\quad[0,1,0,1,0] \oplus[2,0,0,1,0] \oplus[3,0,0,0,1] \oplus[1,1,0,0,1] \oplus[1,0,0,3,0] \oplus$ $[4,0,0,1,0] \oplus[0,2,0,1,0] \oplus[1,0,1,1,0] \oplus(2)[2,1,0,1,0] \oplus[5,0,0,0,1] \oplus[2,0,1,0,1] \oplus$ $[1,2,0,0,1] \oplus(2)[3,1,0,0,1] \oplus[2,0,0,2,1] \oplus[3,0,1,1,0] \oplus[4,1,0,1,0] \oplus[1,1,1,1,0] \oplus$ $[2,2,0,1,0] \oplus[3,0,0,1,2] \oplus[4,0,0,0,3] \oplus[5,1,0,0,1] \oplus[4,0,1,0,1] \oplus[3,2,0,0,1] \oplus$ $[2,1,1,0,1]$ 
- Level-6: $\quad[0,0,1,0,0] \oplus[1,1,0,0,0] \oplus[1,0,0,1,1] \oplus[0,1,1,0,0] \oplus[0,1,0,2,0] \oplus$ (2) $[2,0,1,0,0] \oplus[1,2,0,0,0] \oplus[3,1,0,0,0] \oplus[2,0,0,2,0] \oplus(2)[3,0,0,1,1] \oplus[5,1,0,0,0] \oplus$ $[0,2,1,0,0] \oplus(2)[1,1,0,1,1] \oplus[1,3,0,0,0] \oplus(2)[4,0,1,0,0] \oplus[1,0,1,2,0] \oplus[3,2,0,0,0] \oplus$ $[4,0,0,0,2] \oplus(2)[2,1,1,0,0] \oplus[2,1,0,2,0] \oplus[2,1,0,0,2] \oplus[5,0,0,1,1] \oplus[6,0,1,0,0] \oplus$ $[2,0,1,1,1] \oplus[1,2,0,1,1] \oplus(2)[3,1,0,1,1] \oplus[4,1,1,0,0] \oplus[2,2,1,0,0] \oplus[3,0,1,0,2] \oplus$ $[4,1,0,0,2]$

- Level-7: $\quad[0,0,0,0,1] \oplus[1,0,0,1,0] \oplus[0,1,0,0,1] \oplus[2,0,0,0,1] \oplus[0,0,1,1,0] \oplus$ $[3,0,0,1,0] \oplus(2)[1,1,0,1,0] \oplus[4,0,0,0,1] \oplus[0,2,0,0,1] \oplus[1,0,1,0,1] \oplus[1,0,0,2,1] \oplus$ $(2)[2,1,0,0,1] \oplus[5,0,0,1,0] \oplus[0,1,1,1,0] \oplus(2)[2,0,1,1,0] \oplus(2)[1,2,0,1,0] \oplus$ (2) $[3,1,0,1,0] \oplus[6,0,0,0,1] \oplus[2,0,0,1,2] \oplus[0,3,0,0,1] \oplus[7,0,0,1,0] \oplus(2)[3,0,1,0,1] \oplus$ $(2)[4,1,0,0,1] \oplus[1,1,1,0,1] \oplus(2)[2,2,0,0,1] \oplus[3,0,0,2,1] \oplus[1,1,0,2,1] \oplus[5,1,0,1,0] \oplus$ $[1,3,0,1,0] \oplus[4,0,1,1,0] \oplus[3,2,0,1,0] \oplus[4,0,0,1,2] \oplus[2,1,1,1,0] \oplus[2,1,0,1,2] \oplus$ $[5,0,1,0,1] \oplus[3,1,1,0,1]$

- Level-8: $\quad[0,0,0,0,0] \oplus[0,1,0,0,0] \oplus[2,0,0,0,0] \oplus[0,0,0,1,1] \oplus[4,0,0,0,0] \oplus$ $[0,2,0,0,0] \oplus[1,0,1,0,0] \oplus[1,0,0,2,0] \oplus[1,0,0,0,2] \oplus[2,1,0,0,0] \oplus[0,0,2,0,0] \oplus$ $[6,0,0,0,0] \oplus[0,1,0,1,1] \oplus[0,3,0,0,0] \oplus(2)[2,0,0,1,1] \oplus[4,1,0,0,0] \oplus[3,0,1,0,0] \oplus$ $(2)[2,2,0,0,0] \oplus[3,0,0,2,0] \oplus[3,0,0,0,2] \oplus(2)[1,1,1,0,0] \oplus[8,0,0,0,0] \oplus[1,1,0,2,0] \oplus$ $[1,1,0,0,2] \oplus[0,4,0,0,0] \oplus[6,1,0,0,0] \oplus[1,0,1,1,1] \oplus[0,2,0,1,1] \oplus(2)[4,0,0,1,1] \oplus$ $[5,0,1,0,0] \oplus[2,0,2,0,0] \oplus[4,2,0,0,0] \oplus[2,3,0,0,0] \oplus[5,0,0,2,0] \oplus[5,0,0,0,2] \oplus$ (3) $[2,1,0,1,1] \oplus[1,2,1,0,0] \oplus(2)[3,1,1,0,0] \oplus[1,2,0,2,0] \oplus[1,2,0,0,2] \oplus[2,0,0,2,2] \oplus$ $[3,1,0,2,0] \oplus[3,1,0,0,2] \oplus[6,0,0,1,1] \oplus[4,0,2,0,0] \oplus[3,0,1,1,1] \oplus[4,1,0,1,1] \oplus$ $[2,2,0,1,1]$

- Level-9: $\quad[0,0,0,1,0] \oplus[1,0,0,0,1] \oplus[0,1,0,1,0] \oplus[2,0,0,1,0] \oplus[0,0,1,0,1] \oplus$ $[3,0,0,0,1] \oplus(2)[1,1,0,0,1] \oplus[4,0,0,1,0] \oplus[0,2,0,1,0] \oplus[1,0,1,1,0] \oplus[1,0,0,1,2] \oplus$ $(2)[2,1,0,1,0] \oplus[5,0,0,0,1] \oplus[0,1,1,0,1] \oplus(2)[2,0,1,0,1] \oplus(2)[1,2,0,0,1] \oplus$ $(2)[3,1,0,0,1] \oplus[6,0,0,1,0] \oplus[2,0,0,2,1] \oplus[0,3,0,1,0] \oplus[7,0,0,0,1] \oplus(2)[3,0,1,1,0] \oplus$ $(2)[4,1,0,1,0] \oplus[1,1,1,1,0] \oplus(2)[2,2,0,1,0] \oplus[3,0,0,1,2] \oplus[1,1,0,1,2] \oplus[5,1,0,0,1] \oplus$ $[1,3,0,0,1] \oplus[4,0,1,0,1] \oplus[3,2,0,0,1] \oplus[4,0,0,2,1] \oplus[2,1,1,0,1] \oplus[2,1,0,2,1] \oplus$ $[5,0,1,1,0] \oplus[3,1,1,1,0]$

- Level-10: $\quad[0,0,1,0,0] \oplus[1,1,0,0,0] \oplus[1,0,0,1,1] \oplus[0,1,1,0,0] \oplus[0,1,0,0,2] \oplus$ $(2)[2,0,1,0,0] \oplus[1,2,0,0,0] \oplus[3,1,0,0,0] \oplus[2,0,0,0,2] \oplus(2)[3,0,0,1,1] \oplus[5,1,0,0,0] \oplus$ $[0,2,1,0,0] \oplus(2)[1,1,0,1,1] \oplus[1,3,0,0,0] \oplus(2)[4,0,1,0,0] \oplus[1,0,1,0,2] \oplus[3,2,0,0,0] \oplus$ $[4,0,0,2,0] \oplus(2)[2,1,1,0,0] \oplus[2,1,0,2,0] \oplus[2,1,0,0,2] \oplus[5,0,0,1,1] \oplus[6,0,1,0,0] \oplus$ $[2,0,1,1,1] \oplus[1,2,0,1,1] \oplus(2)[3,1,0,1,1] \oplus[4,1,1,0,0] \oplus[2,2,1,0,0] \oplus[3,0,1,2,0] \oplus$ $[4,1,0,2,0]$

- Level-11: $\quad[0,1,0,0,1] \oplus[2,0,0,0,1] \oplus[3,0,0,1,0] \oplus[1,1,0,1,0] \oplus[1,0,0,0,3] \oplus$ $[4,0,0,0,1] \oplus[0,2,0,0,1] \oplus[1,0,1,0,1] \oplus(2)[2,1,0,0,1] \oplus[5,0,0,1,0] \oplus[2,0,1,1,0] \oplus$ $[1,2,0,1,0] \oplus(2)[3,1,0,1,0] \oplus[2,0,0,1,2] \oplus[3,0,1,0,1] \oplus[4,1,0,0,1] \oplus[1,1,1,0,1] \oplus$ $[2,2,0,0,1] \oplus[3,0,0,2,1] \oplus[4,0,0,3,0] \oplus[5,1,0,1,0] \oplus[4,0,1,1,0] \oplus[3,2,0,1,0] \oplus$ $[2,1,1,1,0]$ 
- Level-12: $\quad[4,0,0,0,0] \oplus[0,2,0,0,0] \oplus[1,0,0,0,2] \oplus[2,1,0,0,0] \oplus[2,0,0,1,1] \oplus$ $[4,1,0,0,0] \oplus[3,0,1,0,0] \oplus[2,2,0,0,0] \oplus[3,0,0,2,0] \oplus[3,0,0,0,2] \oplus[1,1,1,0,0] \oplus$ $[1,1,0,0,2] \oplus[4,0,0,1,1] \oplus[2,0,2,0,0] \oplus[4,2,0,0,0] \oplus[5,0,0,2,0] \oplus[2,1,0,1,1] \oplus$ $[3,1,1,0,0] \oplus[3,1,0,2,0]$

- Level-13: $\quad[3,0,0,0,1] \oplus[1,1,0,0,1] \oplus[4,0,0,1,0] \oplus[2,1,0,1,0] \oplus[2,0,1,0,1] \oplus$ $[3,1,0,0,1] \oplus[3,0,1,1,0] \oplus[4,1,0,1,0]$

- Level-14: $[2,0,1,0,0] \oplus[3,1,0,0,0] \oplus[3,0,0,1,1] \oplus[4,0,1,0,0]$

- Level-15: $[3,0,0,1,0] \oplus[4,0,0,0,1]$

- Level-16: $[4,0,0,0,0]$

Open Access. This article is distributed under the terms of the Creative Commons Attribution License (CC-BY 4.0), which permits any use, distribution and reproduction in any medium, provided the original author(s) and source are credited.

\section{References}

[1] P.S. Howe, Super Weyl Transformations in Two-Dimensions, J. Phys. A 12 (1979) 393 [INSPIRE].

[2] P.S. Howe and R.W. Tucker, Scale Invariance in Superspace, Phys. Lett. B 80 (1978) 138 [INSPIRE].

[3] S.J. Gates Jr., "Superconformal" Symmetry Breakdown As A Guide To Supergravity Constraints, talk given at the Supergravity Workshop, Stony Brook, NY, U.S.A., 27-28 September 1979, HUTP-79/A069 (1979) [INSPIRE].

[4] S.J. Gates Jr., K.S. Stelle and P.C. West, Algebraic Origins of Superspace Constraints in Supergravity, Nucl. Phys. B 169 (1980) 347 [INSPIRE].

[5] S.J. Gates Jr., Supercovariant Derivatives, Super Weyl Groups, and $N=2$ Supergravity, Nucl. Phys. B 176 (1980) 397 [inSPIRE].

[6] S.J. Gates Jr. and W. Siegel, Understanding Constraints in Superspace Formulations of Supergravity, Nucl. Phys. B 163 (1980) 519 [INSPIRE].

[7] S.J. Gates Jr. and H. Nishino, Realization of space-time conformal symmetry in $D=10$, $N=1$ superspace, Phys. Lett. B 266 (1991) 14 [INSPIRE].

[8] E. Cremmer and S. Ferrara, Formulation of Eleven-Dimensional Supergravity in Superspace, Phys. Lett. B 91 (1980) 61 [INSPIRE].

[9] L. Brink and P.S. Howe, Eleven-Dimensional Supergravity on the Mass-Shell in Superspace, Phys. Lett. B 91 (1980) 384 [InSPIRE].

[10] E. Witten, String theory dynamics in various dimensions, Nucl. Phys. B 443 (1995) 85 [hep-th/9503124] [INSPIRE].

[11] P.S. Howe, Weyl superspace, Phys. Lett. B 415 (1997) 149 [hep-th/9707184] [INSPIRE].

[12] M. Cederwall, U. Gran, M. Nielsen and B.E.W. Nilsson, Manifestly supersymmetric M-theory, JHEP 10 (2000) 041 [hep-th/0007035] [INSPIRE]. 
[13] P.S. Howe, S.F. Kerstan, U. Lindström and D. Tsimpis, The Deformed M2-brane, JHEP 09 (2003) 013 [hep-th/0307072] [INSPIRE].

[14] P.S. Howe and D. Tsimpis, On higher order corrections in M-theory, JHEP 09 (2003) 038 [hep-th/0305129] [INSPIRE].

[15] E. Bergshoeff and M. de Roo, The Supercurrent in Ten-dimensions, Phys. Lett. B 112 (1982) 53 [INSPIRE].

[16] E. Bergshoeff, M. de Roo and B. de Wit, Conformal Supergravity in Ten-dimensions, Nucl. Phys. B 217 (1983) 489 [inSPIRE].

[17] K. Peeters, P. Vanhove and A. Westerberg, Supersymmetric higher derivative actions in ten-dimensions and eleven-dimensions, the associated superalgebras and their formulation in superspace, Class. Quant. Grav. 18 (2001) 843 [hep-th/0010167] [INSPIRE].

[18] K. Peeters, P. Vanhove and A. Westerberg, Supersymmetric $R^{4}$ actions and quantum corrections to superspace torsion constraints, in proceedings of the NATO Advanced Research Workshop on Noncommutative Structures in Mathematics and Physics, Kiev, Ukraine, 24-27 September 2000, in proceedings of the International Conference on Quantization, Gauge Theory, and Strings: Conference Dedicated to the Memory of Professor Efim Fradkin, Moscow, Russian Federation, 5-10 June 2000, in proceedings of the Nordic Network on String Theory, Copenhagen, Denmark, 4-6 May 2000, hep-th/0010182 [INSPIRE].

[19] K. Peeters, P. Vanhove and A. Westerberg, Towards complete string effective actions beyond leading order, Fortsch. Phys. 52 (2004) 630 [hep-th/0312211] [INSPIRE].

[20] H. Nishino and S.J. Gates Jr., Toward an off-shell $11 D$ supergravity limit of M-theory, Phys. Lett. B 388 (1996) 504 [hep-th/9602011] [INSPIRE].

[21] S.J. Gates Jr., Superconformal symmetry in $11 D$ superspace and the M-theory effective action, Nucl. Phys. B 616 (2001) 85 [hep-th/0106150] [INSPIRE].

[22] S.J. Gates Jr., Y. Hu, H. Jiang and S.-N. Hazel Mak, A codex on linearized Nordström supergravity in eleven and ten dimensional superspaces, JHEP 07 (2019) 063 [arXiv: 1812.05097] [INSPIRE].

[23] S.J. Gates Jr., Y. Hu and S.-N. Hazel Mak, Superfield Component Decompositions and the Scan for Prepotential Supermultiplets in 10D Superspaces, JHEP 02 (2020) 176 [arXiv: 1911.00807] [INSPIRE].

[24] S.J. Gates Jr., Y. Hu and S.-N. Hazel Mak, Adinkra foundation of component decomposition and the scan for superconformal multiplets in $11 D, \mathcal{N}=1$ superspace, JHEP 09 (2020) 089 [arXiv: 2002.08502] [INSPIRE].

[25] S.J. Gates Jr., Y. Hu and S.-N. Hazel Mak, Advening to Adynkrafields: Young Tableaux to Component Fields of the 10D, $N=1$ Scalar Superfield, arXiv:2006.03609 [INSPIRE].

[26] S.J. Gates Jr. and W. Siegel, Linearized N=2 Superfield Supergravity, Nucl. Phys. B 195 (1982) 39 [INSPIRE].

[27] R. Grimm, J. Wess and B. Zumino, Consistency Checks on the Superspace Formulation of Supergravity, Phys. Lett. B 73 (1978) 415 [inSPIRE].

[28] R. Grimm, J. Wess and B. Zumino, A Complete Solution of the Bianchi Identities in Superspace, Nucl. Phys. B 152 (1979) 255 [InSPIRE]. 
[29] M. Cederwall, Pure spinor superfields - an overview, in Breaking of Supersymmetry and Ultraviolet Divergences in Extended Supergravity, Springer Proceedings in Physics 153, Springer, Cham Switzerland (2014), pp. 61-93 [arXiv:1307.1762] [INSPIRE].

[30] R.L. Arnowitt, S. Deser and C.W. Misner, Dynamical Structure and Definition of Energy in General Relativity, Phys. Rev. 116 (1959) 1322 [INSPIRE].

[31] S. Deser and C.J. Isham, Canonical Vierbein Form of General Relativity, Phys. Rev. D 14 (1976) 2505 [INSPIRE].

[32] J.A. Nester, A New gravitational energy expression with a simple positivity proof, Phys. Lett. A 83 (1981) 241 [INSPIRE].

[33] S.J. Gates Jr. and H. Nishino, $D=2$ superfield supergravity, local (supersymmetry) ${ }^{2}$ and non-linear $\Sigma$ models, in proceedings of the Miniworkshop on Superstrings and All That, Blacksburg, VA, U.S.A., 17-21 June 1985, Class. Quant. Grav. 3 (1986) 391 [INSPIRE].

[34] S.J. Gates Jr. and H. Nishino, Deliberations on $11 D$ superspace for the M-theory effective action, Phys. Lett. B 508 (2001) 155 [hep-th/0101037] [INSPIRE].

[35] R.M. Fonseca, Calculating the renormalisation group equations of a SUSY model with Susyno, Comput. Phys. Commun. 183 (2012) 2298 [arXiv:1106.5016] [InSPIRE].

[36] M.A.A. Van Leeuwen, LiE, a software package for Lie group computations, Euromath Bull. 1 (1994) 83.

[37] R. Feger and T.W. Kephart, LieART - A Mathematica application for Lie algebras and representation theory, Comput. Phys. Commun. 192 (2015) 166 [arXiv:1206.6379] [INSPIRE].

[38] https://blast.ncbi.nlm.nih.gov/Blast.cgi.

[39] S.J. Gates Jr. and R. Grimm, Consequences of Conformally Covariant Constraints for $\mathcal{N}>4$ Superspace, Phys. Lett. B 133 (1983) 192 [INSPIRE].

[40] S.J. Gates Jr. et al., 4D, $\mathcal{N}=1$ Supersymmetry Genomics (I), JHEP 12 (2009) 008 [arXiv:0902.3830] [INSPIRE].

[41] W. Siegel, Unextended Superfields in Extended Supersymmetry, Nucl. Phys. B 156 (1979) 135 [INSPIRE].

[42] S.J. Gates Jr., Toward an Unextended Superfield Formulation of $\mathcal{N}=2$ Supergravity, talk given at the Nuffield Workshop on Superspace and Supergravity, Cambridge, U.K., 16 June-12 July 1980, HUTP-80/A055 (1980) [inSPIRE].

[43] S.J. Gates Jr., A. Karlhede, U. Lindström and M. Roček, N=1 Superspace Components of Extended Supergravity, Class. Quant. Grav. 1 (1984) 227 [InSPIRE].

[44] S.J. Gates Jr., A. Karlhede, U. Lindström and M. Roček, N=1 Superspace Geometry of Extended Supergravity, Nucl. Phys. B 243 (1984) 221 [INSPIRE].

[45] J.M.F. Labastida, M. Roček, E. Sanchez-Velasco and P. Wills, $N=2$ Supergravity Action in Terms of $N=1$ Superfields, Phys. Lett. B 151 (1985) 111 [InSPIRE].

[46] K. Becker and D. Butter, 4D N=1 Kaluza-Klein superspace, JHEP 09 (2020) 091 [arXiv:2003.01790] [INSPIRE].

[47] K. Becker, M. Becker, S. Guha, W.D. Linch and D. Robbins, M-theory potential from the $G_{2}$ Hitchin functional in superspace, JHEP 12 (2016) 085 [arXiv: 1611.03098] [INSPIRE]. 
[48] K. Becker, M. Becker, D. Butter, S. Guha, W.D. Linch and D. Robbins, Eleven-dimensional supergravity in 4D, $N=1$ superspace, JHEP 11 (2017) 199 [arXiv:1709.07024] [INSPIRE].

[49] K. Becker, M. Becker, D. Butter and W.D. Linch, $N=1$ supercurrents of eleven-dimensional supergravity, JHEP 05 (2018) 128 [arXiv:1803.00050] [INSPIRE].

[50] A.H. Chamseddine, Interacting Supergravity in Ten-Dimensions: The Role of the Six-Index Gauge Field, Phys. Rev. D 24 (1981) 3065 [INSPIRE].

[51] E. Bergshoeff, M. de Roo, B. de Wit and P. van Nieuwenhuizen, Ten-Dimensional Maxwell-Einstein Supergravity, Its Currents, and the Issue of Its Auxiliary Fields, Nucl. Phys. B 195 (1982) 97 [INSPIRE].

[52] G.F. Chapline and N.S. Manton, Unification of Yang-Mills Theory and Supergravity in Ten-Dimensions, Phys. Lett. B 120 (1983) 105 [InSPIRE].

[53] J.H. Schwarz, Superstring Theory, Phys. Rept. 89 (1982) 223 [InSPIRE].

[54] M.B. Green, Supersymmetrical Dual String Theories and their Field Theory Limits: A Review, Surveys High Energ. Phys. 3 (1984) 127 [InSPIRE].

[55] D.J. Gross, J.A. Harvey, E.J. Martinec and R. Rohm, The Heterotic String, Phys. Rev. Lett. 54 (1985) 502 [INSPIRE].

[56] S.J. Gates Jr. and H. Nishino, New $D=10, N=1$ Superspace Supergravity and Local Symmetries of Superstrings, Phys. Lett. B 173 (1986) 46 [INSPIRE].

[57] S.J. Gates Jr. and H. Nishino, Manifestly Supersymmetric $O\left(\alpha^{\prime}\right)$ Superstring Corrections in New $D=10, N=1$ Supergravity Yang-Mills Theory, Phys. Lett. B 173 (1986) 52 [InSPIRE].

[58] P.S. Howe, H. Nicolai and A. Van Proeyen, Auxiliary Fields and a Superspace Lagrangian for Linearized Ten-dimensional Supergravity, Phys. Lett. B 112 (1982) 446 [INSPIRE].

[59] S.J. Gates Jr. and S. Vashakidze, On $D=10, N=1$ Supersymmetry, Superspace Geometry and Superstring Effects, Nucl. Phys. B 291 (1987) 172 [INSPIRE]. 